\title{
Development of an Aerostructural Analysis Tool for a Low-Sweep Parametric Wing
}

\author{
Julian Bardin
}

500758483

Presented to Dr. Joon Chung

Ryerson University

April 16, 2021 


\section{Acknowledgements}

The author would like to thank Dr. Joon Chung for providing the opportunity and support to develop this project. The author would also like to thank Pratik Pradhan and Mohsen Rostami for their regular guidance throughout the development of this analysis. The author would lastly like to thank Ryerson University for the chance to create an undergraduate thesis. 


\begin{abstract}
An aerostructural analysis program was developed to predict the aerodynamic performance of a non-rigid, low-sweep wing. The wing planform was geometrically defined to have a rectangular section, and a trapezoidal section. The cross-section was further set to an airfoil shape which was consistent across the entire wingspan. Furthermore, to enable the inclusion of this multidisciplinary analysis module into an optimization scheme, the wing geometry was defined by a series of parameters: root chord, taper ratio, leading-edge sweep, semi-span length, and the kink location.

Aerodynamic analysis was implemented through the quasi-three-dimensional approach, including a threedimensional inviscid solution and a sectional two-dimensional viscous solution. The inviscid analysis was provided through the implementation of the vortex ring lifting surface method, which modelled the wing about its mean camber surface.

The viscous aerodynamic solution was implemented through a sectional slicing of the wing. For each section, the effective angle of attack was determined and provided as an input to a two-dimensional airfoil solver. This airfoil solution was comprised of two subcomponents: a linear-strength vortex method inviscid solution, and a direct-method viscous boundary layer computation. The converged airfoil solution was developed by adjusting the effective airfoil geometry to account for the boundary layer displacement thickness, which in itself required the inviscid tangential speeds to compute.

The structural solution was implemented through classical beam theory, with a torsion and bending calculator included. The torque and bending moment distribution along the wing were computed from the lift distribution, neglecting the effects of drag, and used to compute the twist and deflection of the wing.

Interdisciplinary coupling was achieved through an iterative scheme. With the developed implementation, the inviscid lift loads were used to compute the deformation of the wing. This deformation was used to update the wing mesh, and the inviscid analysis was run again. This iteration was continued until the lift variation between computations was below $0.1 \%$. Once the solution was converged upon by the inviscid and structural solutions, the viscous calculator was run to develop the parasitic drag forces. Once computation had completed, the aerodynamic lift and drag forces were output to mark the completion of execution.
\end{abstract}




\section{Table of Contents}

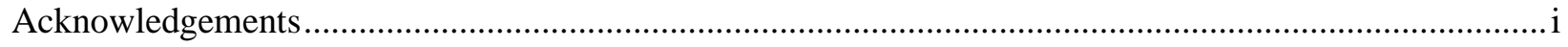

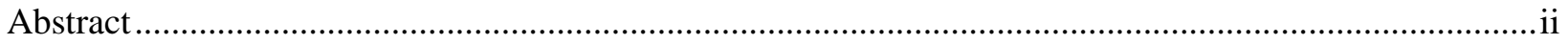

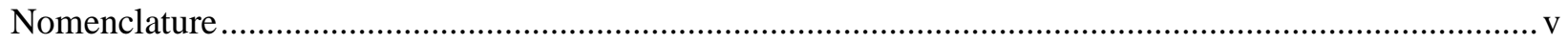

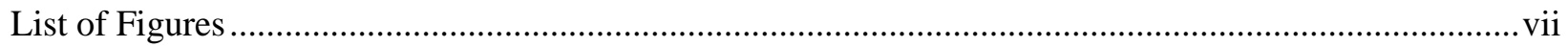

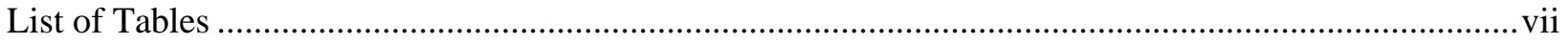

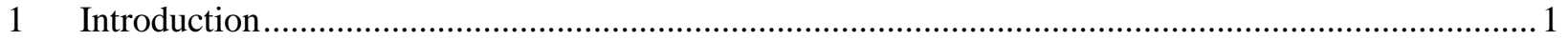

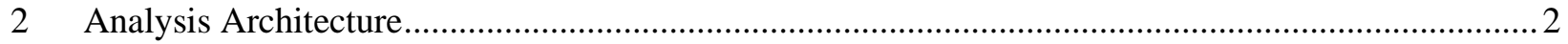

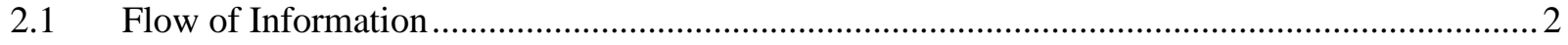

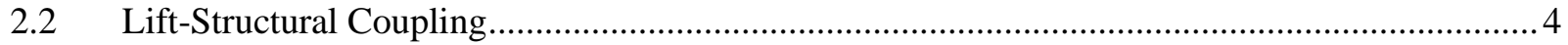

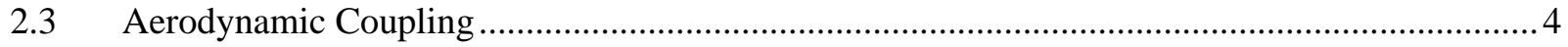

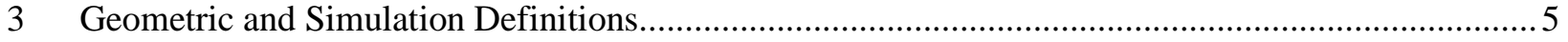

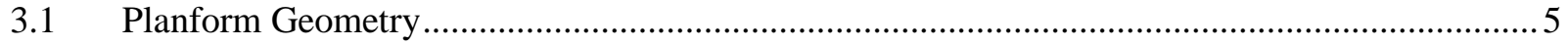

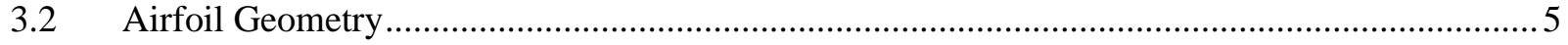

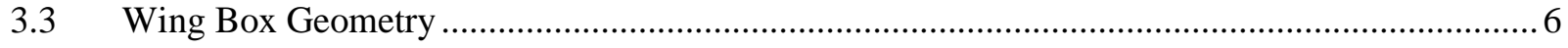

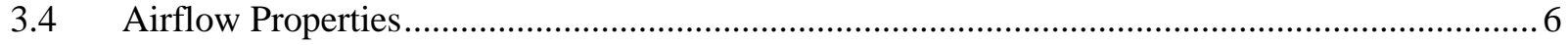

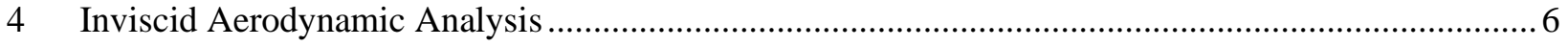

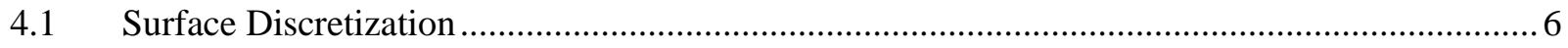

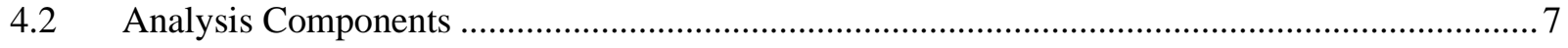

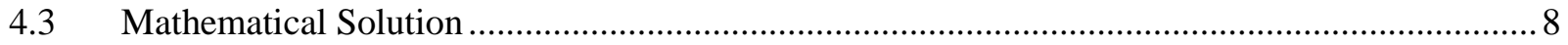

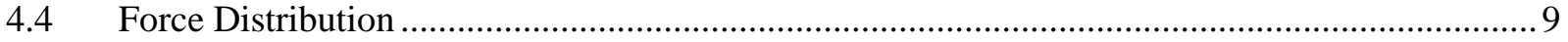

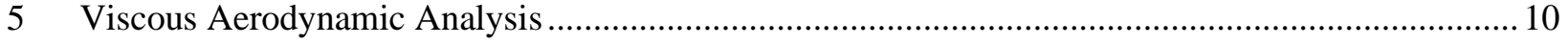

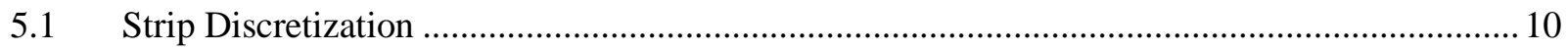

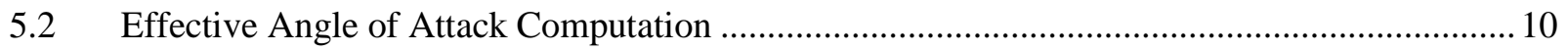

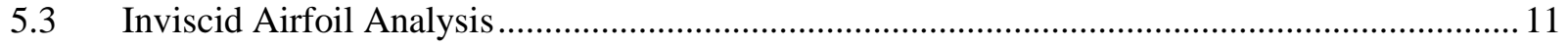

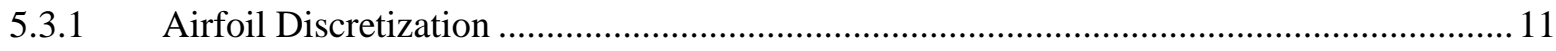

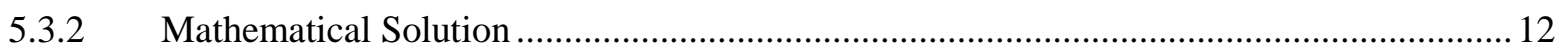

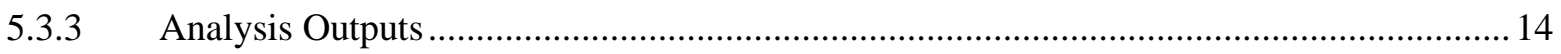

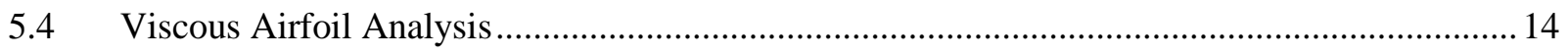

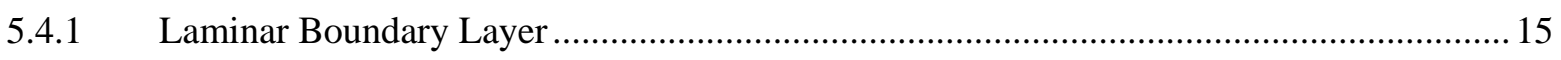

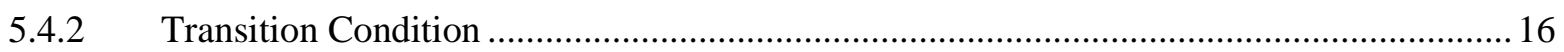

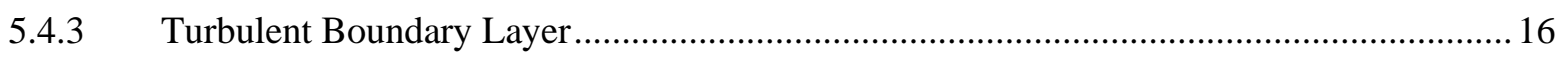

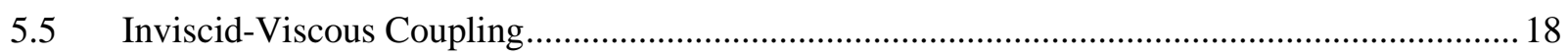

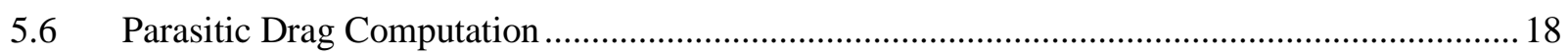

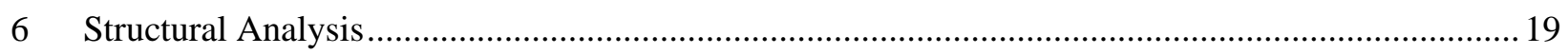




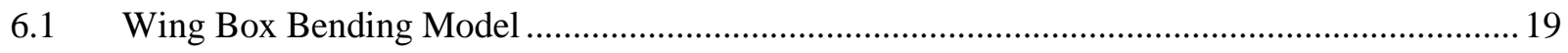

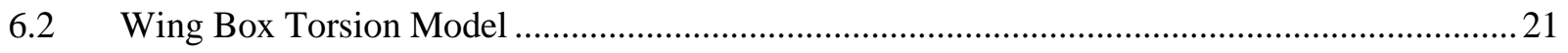

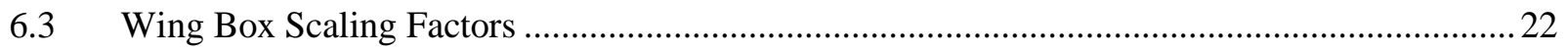

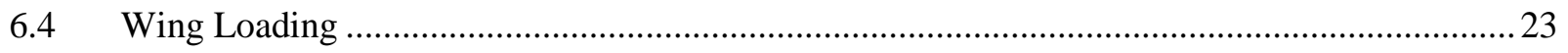

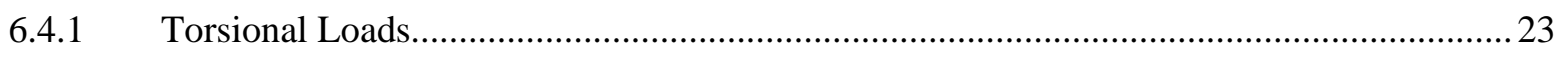

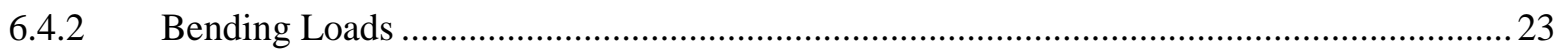

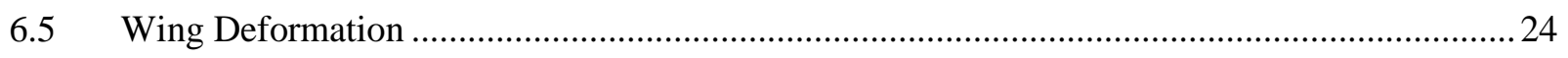

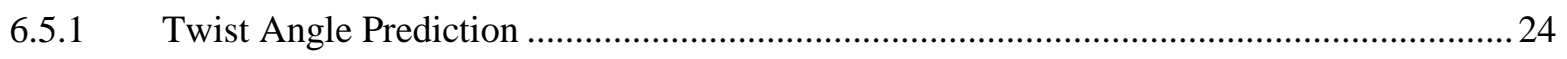

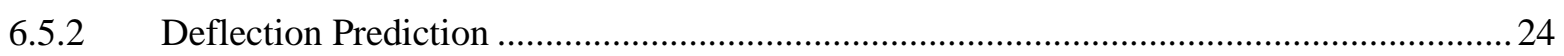

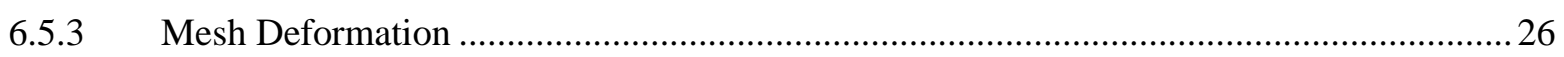

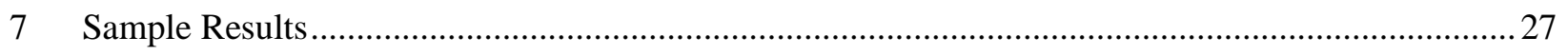

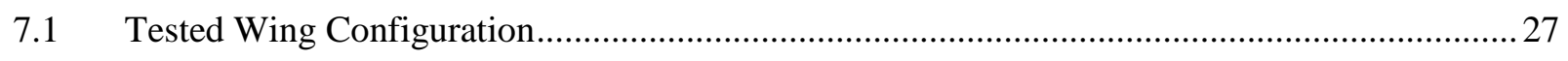

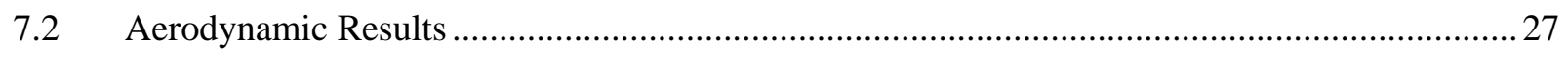

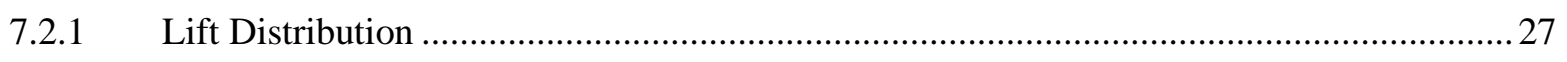

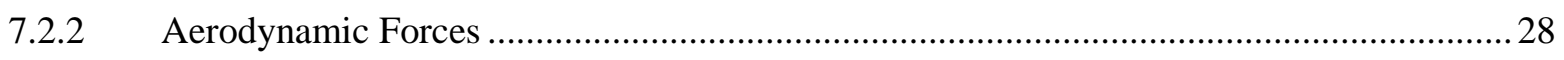

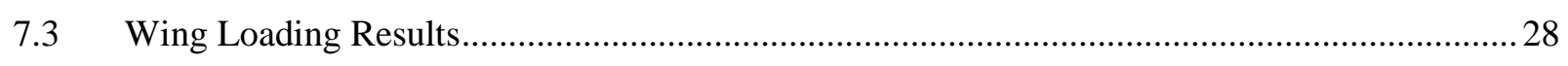

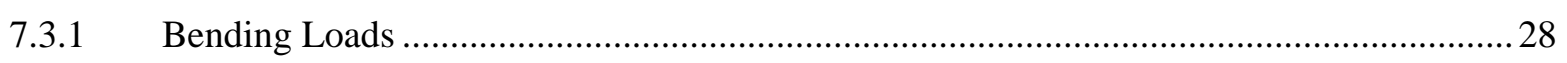

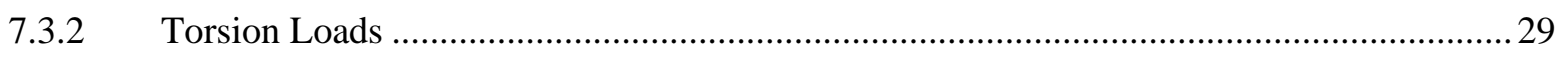

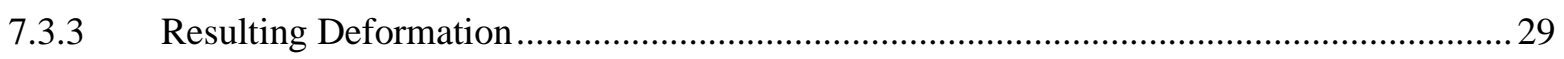

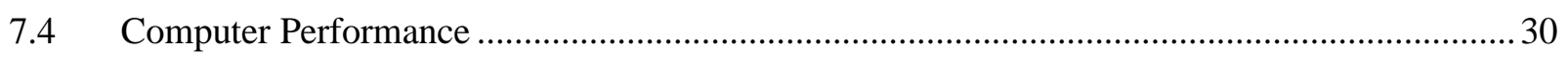

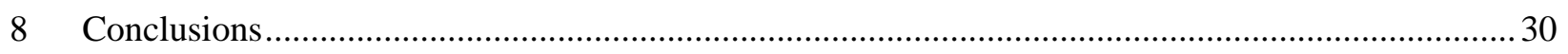

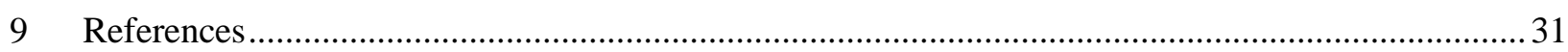

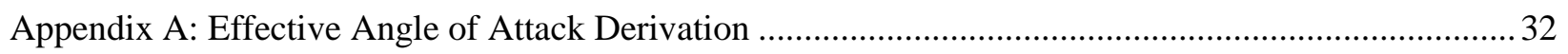

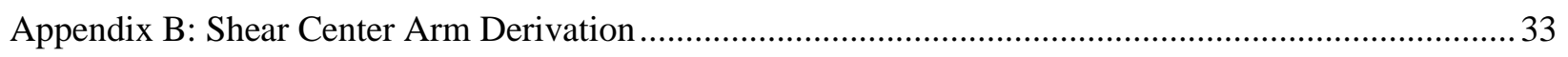

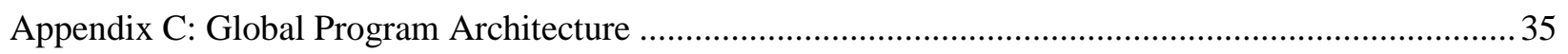

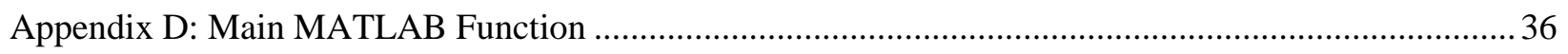

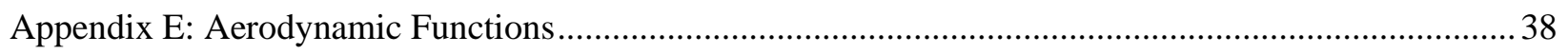

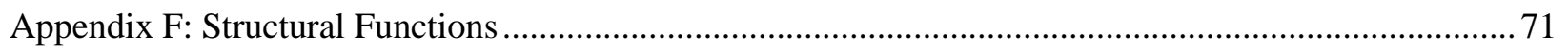

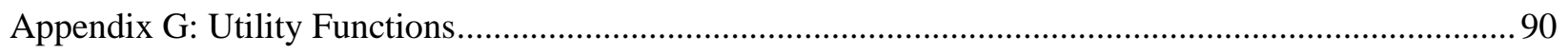




\section{Nomenclature}

Acronyms

$\begin{array}{cl}\text { 2D } & \text { Two-Dimensional } \\ \text { 3D } & \text { Three-Dimensional } \\ \text { CPU } & \text { Central Processing Unit } \\ \text { MATLAB } & \text { Matrix Laboratory } \\ \text { MDA } & \text { Multidisciplinary Analysis } \\ \text { MDF } & \text { Multidisciplinary Feasible } \\ \text { MDO } & \text { Multidisciplinary Optimization } \\ \text { Q3D } & \text { Quasi-Three-Dimensional } \\ \text { RAM } & \text { Random Access Memory } \\ \text { RHS } & \text { Right-Hand-Side, Inviscid Free-Stream Contribution } \\ \text { UTH } & \text { Speed-Theta-Shape Factor, Boundary Layer Analysis } \\ \text { VLM } & \text { Vortex Lattice Method }\end{array}$

Symbols

$\begin{array}{cl}A & \text { Area } \\ \alpha & \text { Angle of Attack } \\ \alpha_{i} & \text { Panel Incidence Angle } \\ a_{K L} & \text { 3D Aerodynamic Influence Coefficient } \\ b_{K L} & \text { 3D Parallel Aerodynamic Influence Coefficient } \\ C_{d i} & \text { Induced Drag Coefficient } \\ C_{f} & \text { Friction Coefficient } \\ C_{l} & \text { Lift Coefficient } \\ C_{l 0} & \text { Zero Angle-of-Attack Lift Coefficient } \\ C_{P} & \text { Pressure Coefficient } \\ c & \text { Chord Length } \\ \Delta L & \text { Panel Lift Force } \\ \Delta D & \text { Panel Drag Force } \\ D_{i} & \text { Induced Drag } \\ D_{p} & \text { Parasitic Drag } \\ d_{i} & \text { Section Induced Drag } \\ d_{p} & \text { Section Parasitic Drag } \\ \delta^{*} & \text { Boundary Layer Displacement Thickness } \\ \varepsilon & \text { Strain } \\ \Gamma & \text { 3D Vorticity } \\ & \end{array}$




\begin{tabular}{|c|c|}
\hline$\gamma$ & 2D Vorticity \\
\hline$H$ & Shape Factor \\
\hline$I_{x x}, I_{z z}$ & Moment of Inertia \\
\hline$I_{Z x}$ & Product of Inertia \\
\hline$L$ & Lift Force \\
\hline$l$ & Section Lift \\
\hline$\lambda$ & Pressure-Gradient Parameter \\
\hline$M$ & Bending Moment \\
\hline$M_{\infty}$ & Free-Stream Mach Number \\
\hline$\phi$ & Twist Angle \\
\hline$p$ & Shear Flow Lever Arm \\
\hline$\vec{Q} \infty$ & Free Stream Velocity Vector \\
\hline$q$ & Induced Velocity \\
\hline$q_{s}$ & Shear Flow \\
\hline$\rho$ & Air Density \\
\hline$\vec{r}$ & Position Vector \\
\hline$R e$ & Reynolds Number \\
\hline$S$ & Shear Force \\
\hline$s$ & Curve Path Distance \\
\hline$\theta$ & Deflection Angle or Boundary Layer Momentum Thickness \\
\hline$\theta_{p}$ & Principal Axis Angle \\
\hline$T$ & Torque \\
\hline$t$ & Thickness \\
\hline$u$ & Longitudinal Velocity Component \\
\hline$u_{e}$ & Inviscid Edge Velocity \\
\hline$V$ & Shear Force \\
\hline$V_{\infty}$ & Free-Stream Speed \\
\hline$w$ & Vertical Velocity Component \\
\hline$w_{\text {ind }}$ & Induced Downwash \\
\hline$x$ & Longitudinal Direction, Parallel to Aircraft Axes \\
\hline$y$ & Lateral Direction, Parallel to Aircraft Axes \\
\hline$Z$ & Vertical Direction, Parallel to Aircraft Axes \\
\hline
\end{tabular}




\section{List of Figures}

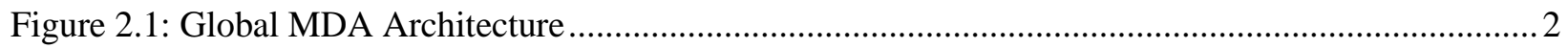

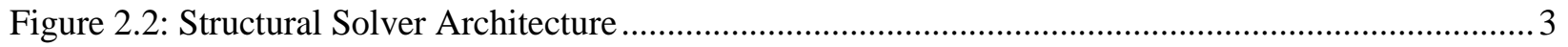

Figure 2.3: Viscous Aerodynamic Solver Architecture ................................................................ 3

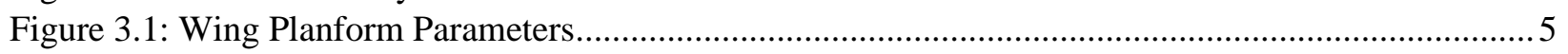

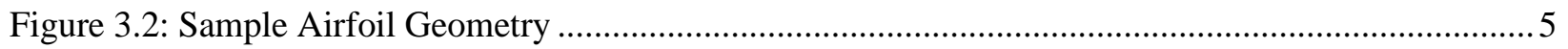

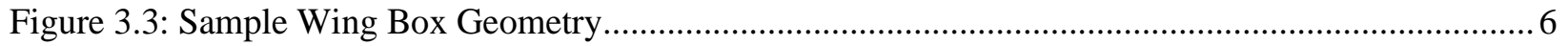

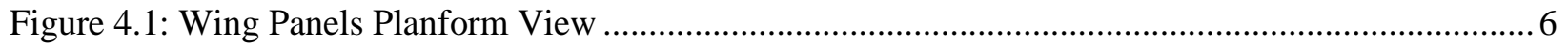

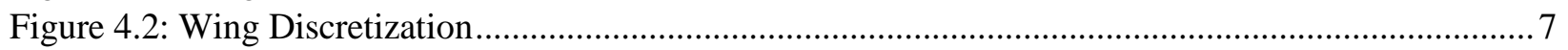

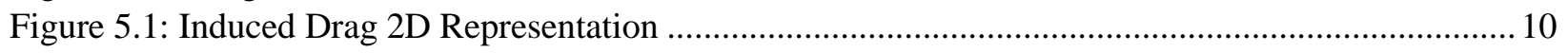

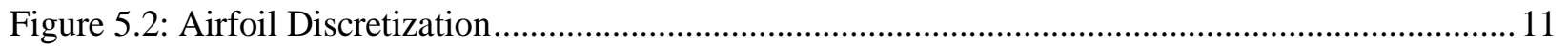

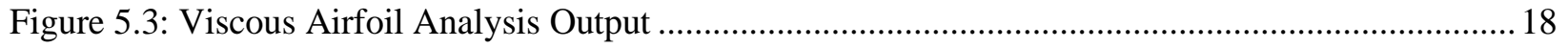

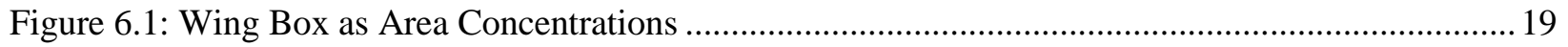

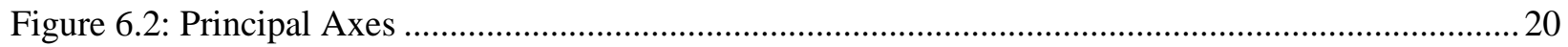

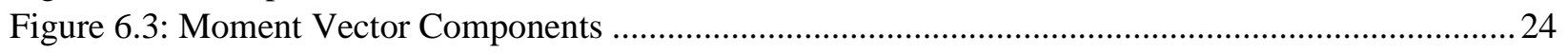

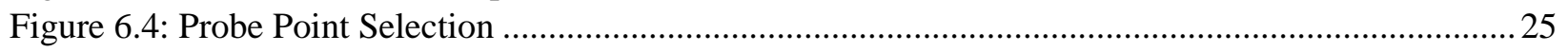

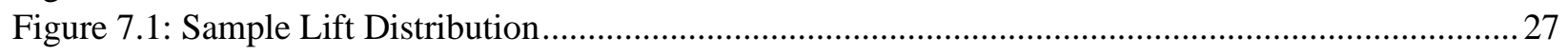

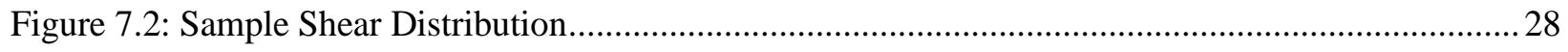

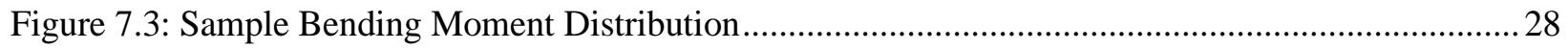

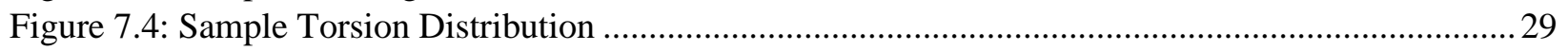

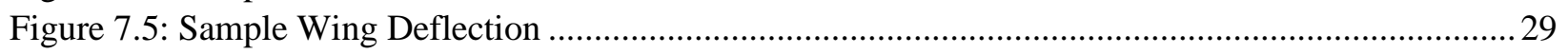

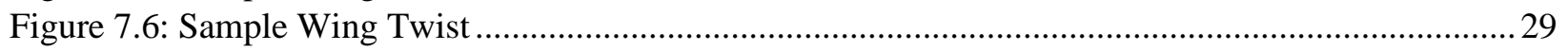

\section{List of Tables}

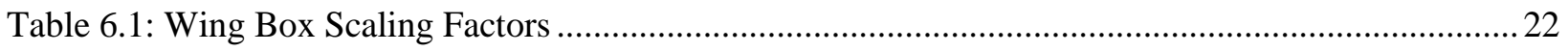

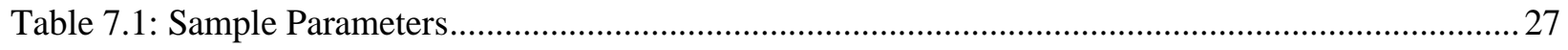

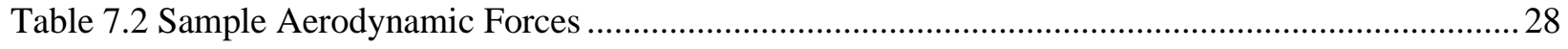

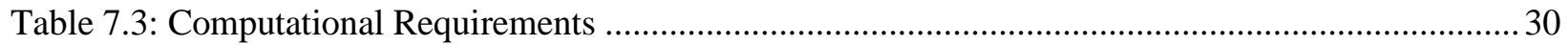




\section{Introduction}

Optimization of aircraft components can be achieved through either a series of single-disciplinary optimization stages, or through a coupled multidisciplinary approach [1]. In pursuing the multidisciplinary approach, designs exhibiting better performance become possible due to an inclusion of the coupled interactions in the analysis [1]. One implementation of this multidisciplinary strategy is the multidisciplinary feasible (MDF) architecture, wherein a multidisciplinary analyser (MDA) is run each time a constraint or objective function is computed [1]. As a precursor to this form of multidisciplinary optimization, an MDA implementation was required.

With a specific focus given to the aerostructural performance of an aircraft wing, a multidisciplinary analysis implementation was developed to predict the resulting aerodynamic performance of a wing when structural deformation was considered. Building off the work of FEMWET by Elham and van Tooren [2], this implementation sought to develop a simplified, thereby faster to compute, aerostructural modelling approach for low-sweep wings. This was comprised of a quasi-three-dimensional (Q3D) aerodynamic solver coupled with a classical bending-torsional structure solver.

The Q3D aerodynamic solver was built utilizing a Vortex Lattice Method (VLM) solver, based on the analysis developed by Katz and Plotkin [3], used to compute the inviscid aerodynamic effects. Viscous effects were subsequently computed using aerodynamic strip theory to divide the wing into a series of sections which were individually analysed [2]. Along each strip, the effective angle of attack was computed from the induced drag, and the acting parasitic drag force was computed iteratively between an inviscid two-dimensional airfoil panel solver and a direct boundary layer approximation. In this implementation, a linear-strength vortex panel method was used to predict the tangential speed distribution along the airfoil surface. This was developed according to the analysis developed by Katz and Plotkin [3]. Using the speed distribution, the resulting boundary layer was computed along the length of the upper and lower airfoil surfaces according to the method presented by Fujiwara et al [4]. The airfoil geometry was adjusted by the boundary layer results, and the converged solution was taken as the parasitic drag acting on the section. The spanwise summation of this was used as the acting parasitic drag for the wing.

The structural solver itself was developed using classical beam theory as presented by Hibbeler [5]. Using this, an implementation was developed where the wing twist about the shear centre was computed, and then the bending deflection of the wing was determined. The combined rotations and deflections along the wing were used to deform the aerodynamic model and adjust the acting loads. For rapid computation, the wing box structure was analysed as a series of area concentrations laid about the wing box frame.

The global aerostructural solution was determined through the coupling of the aerodynamic and structural solvers. To achieve this, the structural effects of the drag was considered negligible and omitted from deforming the wing. An iterative scheme was then employed to apply the loads from the VLM to the structure, which then deformed to adjust the VLM mesh. This process was repeated until the solution had converged, after which the viscous effects were computed, and the multidisciplinary analysis was considered to be complete. 


\section{Analysis Architecture}

\subsection{Flow of Information}

The chosen architecture for the multidisciplinary analysis began first by creating a top-level main function from which the individual analysers were called to produce their respective results. To this end, an encapsulated analysis function was created for each solver, which contained calls for the various subfunctions required to generate the aerodynamic or structural outputs. Additionally, to enable separate iteration schemes, the inviscid aerodynamic solver and the viscous aerodynamic solver were encapsulated separately.

The general flow of information from the main function to and from the encapsulated analysers was presented in Figure 2.1. Inputs to a function have been placed in the same column, while outputs have been placed within the same row [1]. Arrows have also been included to highlight the direction of information.

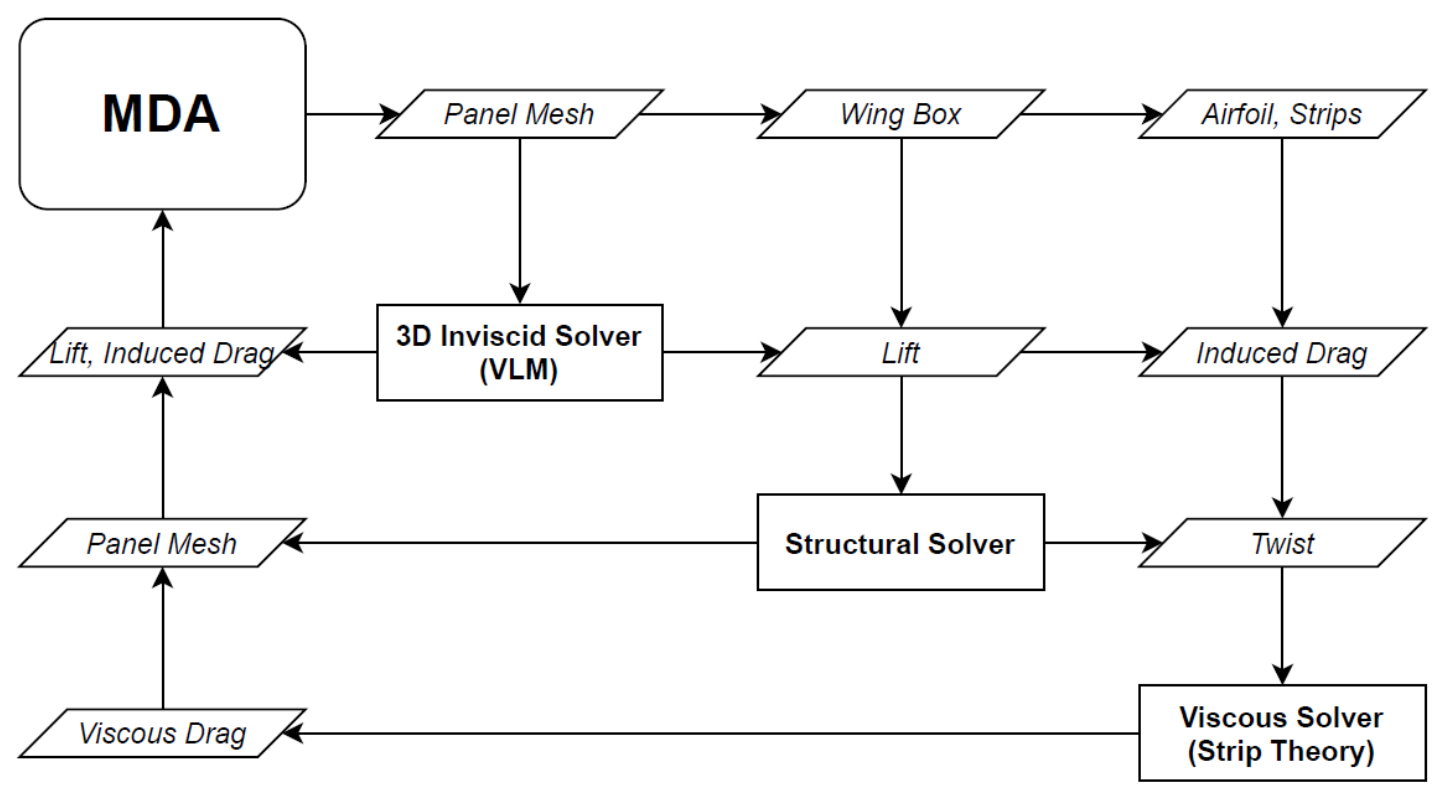

Figure 2.1: Global MDA Architecture

As was shown in the figure, geometric and material properties were sent by the main function into the three solvers. Receiving this information, the solvers would compute their respective outputs. Starting with the inviscid aerodynamic solver, the lift and induced drag distributions along the wing were output. In terms of encapsulation, this was the simplest of the three functions containing only the function calls to populate and solve the aerodynamic influence matrices.

The next solver that was called by the main function was the structural deformation calculator. This did not require any additional internal control logic, and the subfunctions were merely computed in sequence. Internally, this calculator was comprised of two modules: the wing twist solver and the wing deflection solver. First, the torque acting on each section of the wing was used to compute the twist angle at each spanwise location. From there, the bending analysis would determine the deflection through analysing the bending moment at each section and using the twist angle to adjust the location probed for the radius of curvature. 
A third module was categorized within the structural solver but was present within the main analysis function rather than the encapsulated structural solver. This module was the function used to deform the VLM mesh according to the determined bending and twist distribution. This was placed outside of the structural solver to allow for the analysis program to limit the relative change in deformation per iteration.

A visual representation of the structural analysis was presented in Figure 2.2, illustrating the flow of loading, structural, and deformation information through the three sub-modules which comprised this domain.

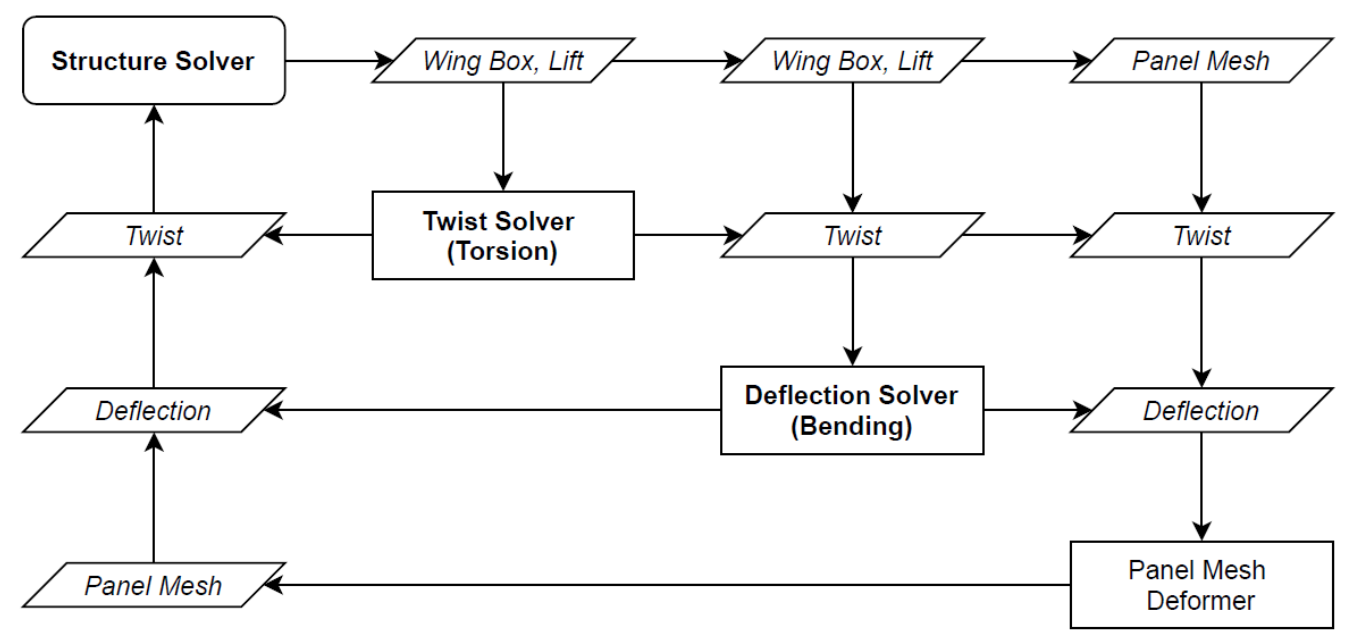

Figure 2.2: Structural Solver Architecture

The last of the three solvers to execute was the viscous drag aerodynamic analysis. Unlike the inviscid aerodynamic and structural analysers, this function contained additional control logic with its own iteration scheme. Four sub-modules comprised this analyser: an effective angle of attack calculator, a 2D inviscid airfoil analyser, a boundary layer solver, and an airfoil geometry manipulator. These modules have been presented in Figure 2.3.

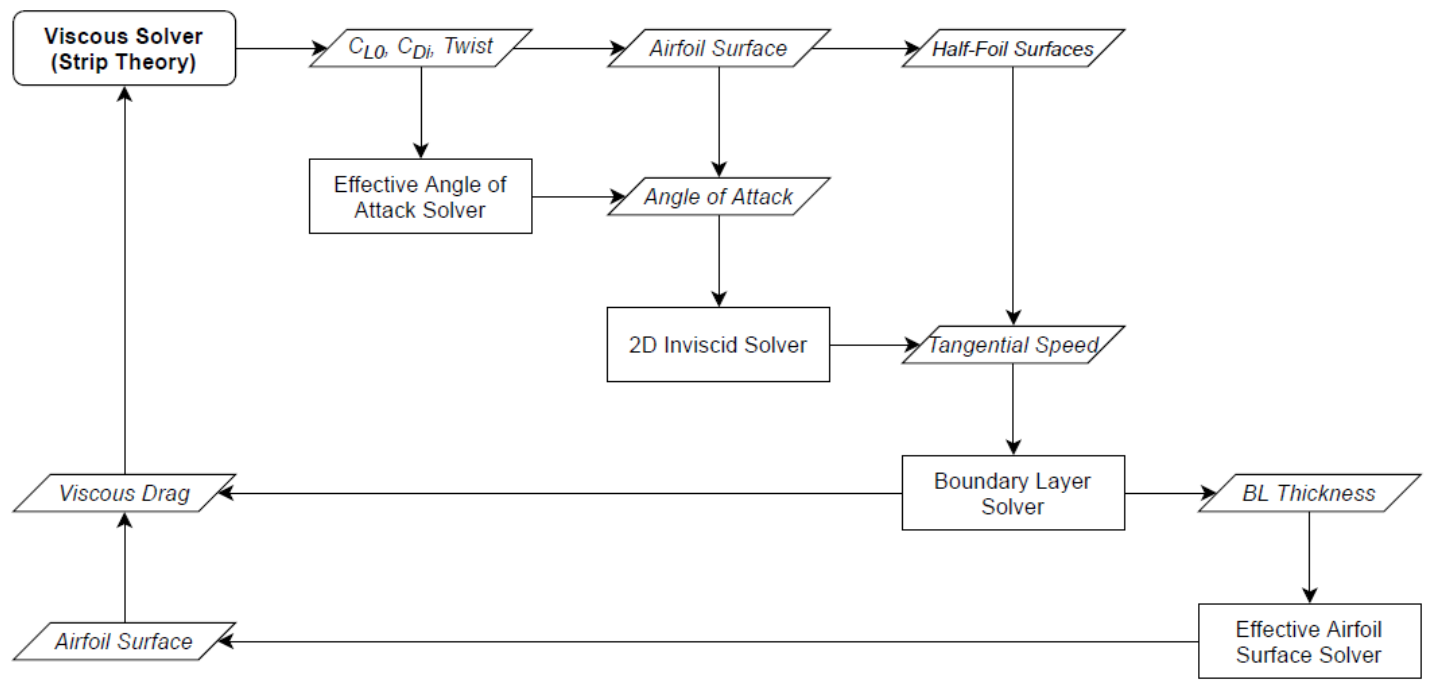

Figure 2.3: Viscous Aerodynamic Solver Architecture 
In using this solver, the wing was divided into a series of strips based on the panel discretization developed for the VLM mesh. For each strip, the effective angle of attack was computed from the induced drag coefficient and a linear-lift approximation. With this parameter determined, an iterative viscous-inviscid coupled 2D aerodynamic analysis was run. With this, the inviscid airfoil panel solver was run to produce a tangential speed distribution along the airfoil surface. This was then used to compute the boundary layer characteristics, including its displacement thickness. The airfoil surface geometry sent to the inviscid calculator was then adjusted with this displacement thickness, and the analysis was run repeatedly until a solution was converged upon [3]. With the solution converged, the sectional viscous drag was returned. This process was repeated for each spanwise section, and the summation of these drag forces was taken as the total parasitic drag acting on the wing.

\subsection{Lift-Structural Coupling}

To simplify the analysis, coupling of the aerodynamic loads with the structural analysis was limited to only the lift loads [2], with the drag loads assumed to have a negligible impact on the wing deformation. With this assumption, the VLM was coupled to the structural solver and the viscous drag calculator was omitted from this stage of the computation. Additionally, while the induced drag was computed by the VLM, its effects too were omitted from structural consideration.

With the coupling mode defined, the iterative scheme was defined with the following steps:

1. Compute the lift force distribution along the wing with the VLM.

2. Compute the deflection and twist along the spanwise direction with the structural solver.

3. Scale the change in twist and deflection for the given iteration (prevented divergent solution)

4. Calculate the percentage change in lift for the given iteration

5. Check if change in lift is below $0.1 \%$. If so, exit the iteration.

6. Otherwise, deform the wing VLM mesh and repeat from Step 1.

The iterative approach was necessitated by the interdependencies present between the aerodynamic and structural solutions. Essentially, the structural solver required the VLM loads as an input while the VLM required the deformed structure as an input. Through the implemented approach, the two solvers were run repeatedly until a final convergent solution was output.

\subsection{Aerodynamic Coupling}

With the two aerodynamic solvers, the flow of information was established to be one-directional. With this implementation, the viscous analysis required the induced drag output by the VLM, but the VLM did not require any input from the viscous solver. Following from this, the viscous analyser was only run a single time for a wing analysis, which was done after the lift-structural coupled solution had been computed. 


\section{Geometric and Simulation Definitions}

To enable the usage of the tool, the wing was defined through a set of parameters. These parameters specified the wing planform, the airfoil geometry, and the wing box characteristics. Additional inputs were given to the analysis which defined the airflow properties.

Furthermore, to retain consistent nomenclature with aircraft, the chordwise direction was given the ' $x$ ' label, the spanwise direction was given the ' $y$ ' label, and the vertical direction was given the ' $z$ ' label. The geometric origin for analysis was placed at the leading edge of the wing root chord.

\subsection{Planform Geometry}

The planform of the wing was defined as having a rectangular section, and a trapezoidal section. The spanwise location where the rectangular section ended was subsequently defined as the "kink" [2]. For the overall wing geometry, the root chord length and semi-span length were used as defining parameters. The location of the kink was defined as a proportion of the semi-span, through the use of a kink distance ratio. For the trapezoidal section, the leading-edge sweep angle was also defined, and the tip chord was computed as the product of the taper ratio and the root chord length. A sample planform with these 5 parameters overlaid was presented in Figure 3.1.

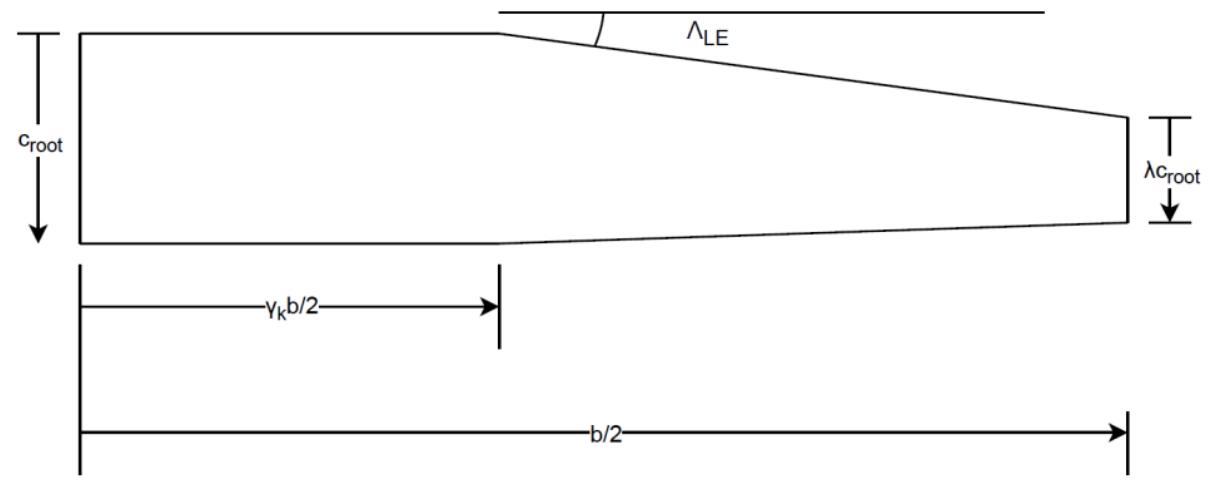

Figure 3.1: Wing Planform Parameters

\subsection{Airfoil Geometry}

The airfoil geometry was defined as a list of points with $x$ and $z$ coordinates [6]. The first point in the array was located at the trailing edge. The subsequent data points defined the curve along the upper surface, then the leading edge, and finally the lower surface [7]. The last point specified in this array was located at the lower surface trailing edge. The geometry of a unit-length airfoil was then defined as the linear interpolation, from the upper trailing edge to the lower trailing edge, of these points. An example of this representation was presented in Figure 3.2, using a NACA 6409 airfoil [6].

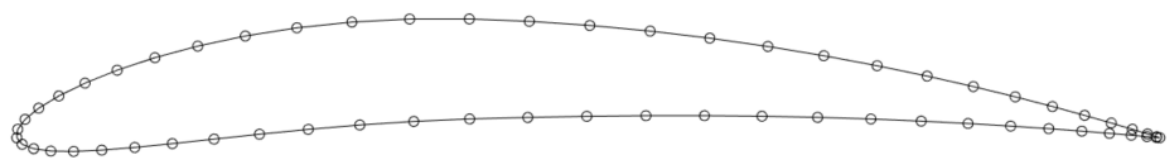

Figure 3.2: Sample Airfoil Geometry 


\subsection{Wing Box Geometry}

The wing box was defined both geometrically and materially. Geometrically, the wing box shape was defined with the airfoil geometry and the placement of the forward and rear spars. The upper and lower surfaces of the wing box were interpolated from the airfoil geometry. The number of longitudinal stiffeners was also included as a wing box defining parameter. The thicknesses of the skin and spars was additionally specified, as well as the cross-sectional area of the stiffeners. For the structural modelling, the elastic modulus and shear modulus were also specified. An example of the geometry resulting from a wing box with spars placed at $20 \%$ and $70 \%$ chord length was presented in Figure 3.3. In this figure, longitudinal stiffeners were represented with dots placed along the skin.

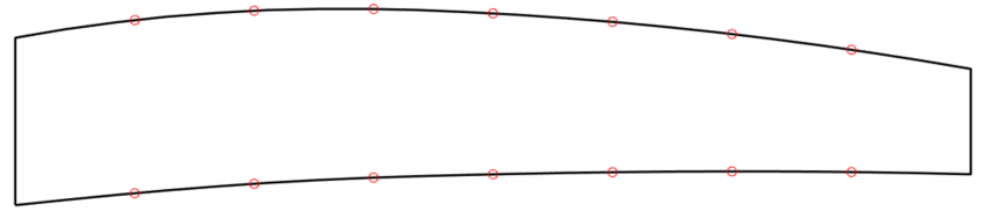

Figure 3.3: Sample Wing Box Geometry

\subsection{Airflow Properties}

A series of additional parameters were defined for the free-stream air properties, which were required to compute the forces acting on the wing. The free-stream true airspeed was defined, as well as the corresponding Mach number. The density and viscosity of air were also specified for the simulation. Finally, and angle of attack was defined.

\section{Inviscid Aerodynamic Analysis}

The inviscid aerodynamic analysis, used to compute the lift and induced drag distribution of the wing, was implemented through the use of a lifting surface solution comprised of vortex ring elements as presented by Katz and Plotkin [2]. This approximation involved modelling the wing as its mean camber surface, imposing discrete panels and vortex rings along it. The solution was then developed and solved using matrix operations [2].

\subsection{Surface Discretization}

In implementing the lifting surface method outlined by Katz and Plotkin [3], the mean camber surface, defined as the surface connecting the mean camber lines of the wing, was approximated by a series of thin panels. Each of these panels was trapezoidal in geometry, with two sides parallel to the free-stream air. A planform view of these trapezoidal panels was provided in Figure 4.1.

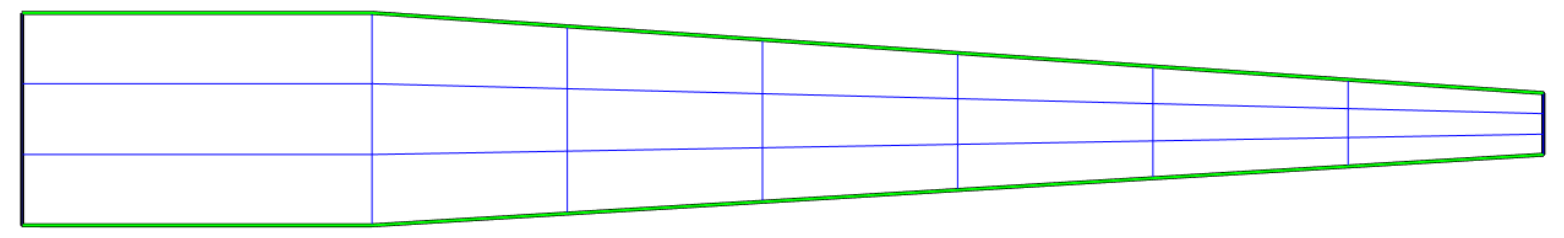

Figure 4.1: Wing Panels Planform View 
As was apparent in Figure 4.1, the panels were arranged to produce a deformed grid. This grid separated the camber surface into a series of sections in the $y$-direction, with each section being further divided by into a set number of individual panels in the $x$-direction.

The $z$-direction coordinates for the panel vertexes were then computed from the mean camber line at each section. To produce a smooth solution, the airfoil geometry was first divided into its upper and lower surfaces. Then, each surface was interpolated from using MATLAB's modified Akima cubic interpolation, chosen as it prevented waving at the leading and trailing edge while still enabling a smooth solution. The mean $z$-coordinate between the upper and lower surface curves was taken to lie on the mean camber line and was used for the panel coordinates.

\subsection{Analysis Components}

Following with the requirements of the vortex ring analysis [3], a corresponding mesh of vortex rings was computed. These were calculated from the panels rather than the mean camber surface and were offset rearward by one-quarter panel length [3] to comply with the two-dimensional Kutta condition. The $z$ direction coordinate for the vortex ring sections behind the wing, caused by the offset, was set to 0 .

An additional row of vortex rings was added to the rear of the mesh in order to model the trailing edge wing wake [3]. The rearmost line of these panels was given an $x$-direction coordinate of 1000000 , considered to be an adequate substitute for infinity.

Additional colocation points were placed on the midpoint of each panel three-quarter chord line [3]. In terms of planform coordinates, while these were placed on the panels, the $x$ - and $y$-coordinates aligned with the centers of the vortex rings. The $z$-coordinate, however, was computed from the panels. These colocation points served as the locations where the vorticities and loads were computed. A unit-length vector normal to each panel surface was placed at these colocation points.

A visual example of the discretized wing mesh was provided with Figure 4.2.

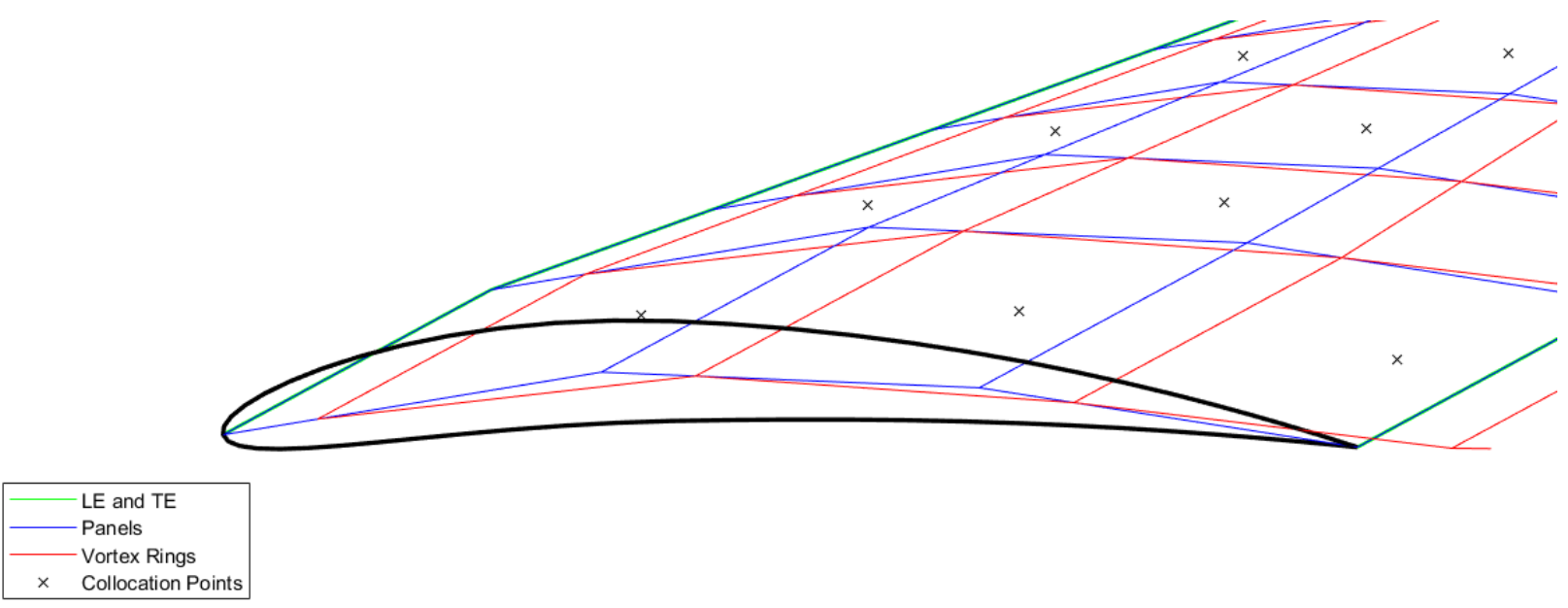

Figure 4.2: Wing Discretization 


\subsection{Mathematical Solution}

With the geometric locations of all simulation components determined, the mathematical definitions were implemented. Globally, the vorticity solution was defined in matrix form with the following equation [3]:

$$
\left[\begin{array}{ccc}
a_{11} & \cdots & a_{1 m} \\
\vdots & \ddots & \vdots \\
a_{m 1} & \cdots & a_{m m}
\end{array}\right]\left[\begin{array}{c}
\Gamma_{1} \\
\vdots \\
\Gamma_{m}
\end{array}\right]=\left[\begin{array}{c}
R H S_{1} \\
\vdots \\
R H S_{m}
\end{array}\right]
$$

Where $a$ was the aerodynamic influence coefficient, $\Gamma$ was the vorticity of the vortex ring, and $R H S$ was the free-stream contribution. Regarding the influence matrix, each row represented the influences acting on a specific colocation point, while the columns referred to the contribution of each colocation to the others. Each coefficient within the aerodynamic influence matrix was computed with the following equation:

$$
a_{K L}=\left(q_{L}+q_{L}^{\prime}+q_{W}+q_{W}^{\prime}\right) \cdot \hat{n}_{K}
$$

Where $a_{K L}$ was the influence coefficient for the $K$ th colocation point and $L$ th vortex ring, $q_{L}$ was the induced velocity at this colocation point caused by the $L$ th vortex ring, $q_{L}^{\prime}$ was the induced velocity by the mirrored vortex ring (as this analysis modeled the right wing, the mirror accounted for the left wing), $q_{W}$ was the induced velocity of the wake, and $q_{W}^{\prime}$ was the induced velocity of the mirrored wake. For non-trailing edge panels, the induced velocity of the wake panels was zero. For all induced velocity computations, a unit vorticity was used. The actual vorticity values were solved with the matrix representation provided in (4.1).

The induced velocity of a vortex ring was computed as the sum of the influences of the individual vortex lines. As each vortex ring was trapezoidal, four vortex lines were summed. The effects of a single vortex line were computed with the following formula [3]:

$$
q=\frac{\Gamma}{4 \pi\left|\overrightarrow{r_{1}} \times \overrightarrow{r_{2}}\right|^{2}}\left(\frac{\overrightarrow{r_{0}} \cdot \overrightarrow{r_{1}}}{\left|\overrightarrow{r_{1}}\right|}-\frac{\overrightarrow{r_{0}} \cdot \overrightarrow{r_{2}}}{\left|\overrightarrow{r_{2}}\right|}\right) \cdot\left(\overrightarrow{r_{1}} \times \overrightarrow{r_{2}}\right)
$$

Where $\overrightarrow{r_{1}}$ was the position vector from the first vortex line point and the colocation point, $\overrightarrow{r_{2}}$ was the position vector from the second vortex line point and the colocation point, and $\overrightarrow{r_{0}}$ was position vector for the vortex line.

Entries within the free-stream contribution matrix were subsequently computed with the following formula [3]:

$$
R H S_{K}=-\vec{Q}_{\infty} \cdot \hat{n}_{K}
$$

Where $\vec{Q}_{\infty}$ was the velocity vector of the free-stream air, accounting for the angle of attack, and $\hat{n}_{K}$ was the unit-length normal vector of each panel. With the aerodynamic influence coefficient matrix and the freestream contribution vector determined, the vorticity of each vortex ring was computed numerically. This was represented in matrix form with the following formula:

$$
[\Gamma]=[A]^{-1}[R H S]
$$

Where $[A]$ was the aerodynamic influence coefficient matrix. 
With the ring vorticities computed, sufficient information had been determined to compute the lift force acting on each panel. Determination of the induced drag, however, required the induced downwash at each colocation points. This was computed according to the following relation [3]:

$$
\left[\begin{array}{c}
w_{\text {ind }, 1} \\
\vdots \\
w_{\text {ind }, m}
\end{array}\right]=\left[\begin{array}{ccc}
b_{11} & \cdots & b_{1 m} \\
\vdots & \ddots & \vdots \\
b_{m 1} & \cdots & b_{m m}
\end{array}\right]\left[\begin{array}{c}
\Gamma_{1} \\
\vdots \\
\Gamma_{m}
\end{array}\right]
$$

Where $w_{\text {ind }}$ was the induced downwash at a colocation point, and $b$ was the component of the aerodynamic influence coefficient determined using only the vortex lines parallel to the free-stream flow.

\subsection{Force Distribution}

The lift and drag force acting on each panel was computed using the vorticity and induced downwash at each colocation point, as well as the free-stream air density and velocity. The formula used for lift computation, which was an implementation of the Kutta-Joukowski theorem, was as follows [3]:

$$
\Delta L_{i j}=\left\{\begin{array}{cc}
\rho Q_{\infty} \Gamma_{i, j} \Delta y_{i j}, & i=1 \\
\rho Q_{\infty}\left(\Gamma_{i, j}-\Gamma_{i-1, j}\right), & i>1
\end{array}\right.
$$

Where $\rho$ was the density in air, $Q_{\infty}$ was the free stream airspeed, $\Delta y$ was the panel width, and the variables $i$ and $j$ were indices. The induced drag was subsequently computed with the following relation [3]:

$$
\Delta D_{i j}=\left\{\begin{array}{c}
-\rho w_{\text {ind }, i j} \Gamma_{i, j} \Delta y_{i j}, i=1 \\
-\rho w_{\text {ind }, i j}\left(\Gamma_{i, j}-\Gamma_{i-1, j}\right), i>1
\end{array}\right.
$$

As the vortex ring analysis was an incompressible analysis, the lift and induced drag were corrected to account for compressible effects. This was done with the Prandtl-Glauert correction, represented with following formulae [3]:

$$
\begin{aligned}
& \Delta L_{i j}^{\prime}=\frac{\Delta L_{i j}}{\sqrt{1-M_{\infty}^{2}}} \\
& \Delta D_{i j}^{\prime}=\frac{\Delta D_{i j}}{\sqrt{1-M_{\infty}^{2}}}
\end{aligned}
$$

Where $\Delta L_{i j}^{\prime}$ was the lift force acting on a panel in compressible flow, $\Delta D_{i j}^{\prime}$ was the induced drag force acting on a panel in compressible flow, and $M_{\infty}$ was the free-stream Mach number. As a final computation for this stage of the analysis, the total lift and induced drag acting on the wing was computed as the sum of the forces acting on the panels, as shown with the following equations [3]:

$$
\begin{gathered}
L=\sum \Delta \mathrm{L}_{\mathrm{ij}}^{\prime} \\
D_{i}=\sum \Delta D_{i j}^{\prime}
\end{gathered}
$$

Where $L$ was the total lift force and $D_{i}$ was the total induced drag. 


\section{Viscous Aerodynamic Analysis}

The viscous aerodynamic analysis of the wing was done through the use of strip theory, where the wing was approximated as a series of spanwise sections, for which 2D effects could be readily modelled [2]. The analysis for each cross section was an iterative viscous-inviscid coupled approach, where an inviscid 2D potential flow analysis was used in conjunction with an empirical viscous drag analysis to converge on a value for parasitic drag.

\subsection{Strip Discretization}

To align with VLM outputs, the wing was cut into a series of strips such that the ends of each strip aligned with the panel mesh boundaries parallel to the free-stream velocity. This was represented in Figure 4.1, where vertical lines of panel boundaries were present, and served as the basis for the sectional analysis. As each mesh section was trapezoidal rather than rectangular, the average chord length for the strip was used.

The inviscid forces, determined through the VLM analysis, acting on this section were used to compute the force coefficients used with the 2D analysis. Specifically, the induced drag coefficient was required for each section to compute the effective angle of attack. This was computed with the following formulae:

$$
\begin{gathered}
d_{i}=\frac{\sqrt{1-M_{\infty}^{2}}}{w} \sum \Delta D_{i}^{\prime} \\
C_{d i}=\frac{2 d_{i}}{\rho V_{\infty}^{2} c}
\end{gathered}
$$

Where $d$ was the induced drag force per unit length, $w$ was the width of the section, $\Delta D_{i}$ was the induced drag acting on each panel within this section, $C_{d i}$ was the 2D induced drag coefficient, $\rho$ was the density of air, and $c$ was the average chord length of the section. The compressibility correction was also removed from the induced drag force computed by the VLM, as the analysis for determining the effective angle of attack required incompressible flow.

\subsection{Effective Angle of Attack Computation}

As a precursor to the inviscid analysis, the effective angle of attack acting on the airfoil was required. This was done to account for the induced drag effects acting on the section, where the downwash produced an effective tilting of the free-stream velocity vector. This, in turn, rotated and altered the acting lift force, allowing a component of the vector to act in the drag direction. The various relevant vectors pertaining to this stage of the analysis were visualized in Figure 5.1.

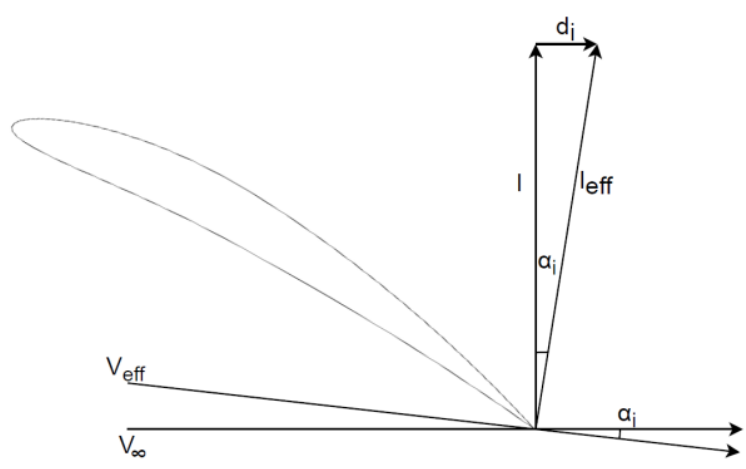

Figure 5.1: Induced Drag 2D Representation 
Utilizing the definitions provided in the previous figure, the effective angle of attack was defined with the following formula:

$$
\alpha_{e f f}=\alpha_{g}+\phi-\alpha_{i}
$$

Where $\alpha_{e f f}$ was the effective angle of attack, $\phi$ was the twist of the section, $\alpha_{g}$ was the global angle of attack of the wing, and $\alpha_{i}$ was the induced angle of attack caused by the downwash. Utilizing the equation determined in Appendix A, including a linear lift assumption, the effective angle of attack was solved numerically using the following relation:

$$
0=\left(2 \pi \alpha_{e f f}+C_{l 0}\right) \sin \left(\alpha_{g}+\phi-\alpha_{e f f}\right)-C_{d i}
$$

Where $C_{l 0}$ was the lift coefficient of the airfoil at zero angle of attack. This formula, using the linearly lift assumption already imposed by the VLM, accounted for both the tilting of the lift vector and its change in magnitude caused by the change in angle of attack. Equation (5.2) was solved numerically using MATLAB's fzero() function, producing a value for the effective angle of attack experienced by the wing strip. This was used as an input for the inviscid airfoil analysis.

\subsection{Inviscid Airfoil Analysis}

The inviscid analysis of the wing section, modelled as a 2D airfoil analysis, was required to compute the tangential speeds along the surface. This was implemented through the use of the linear-strength vortex method defined by Katz and Plotkin [3], similar in execution to XFOIL [7], and generating the solution at the effective angle of attack determined with the model in Section 5.2.

\subsubsection{Airfoil Discretization}

The 2D airfoil geometry was modelled as a series of vortex panels connecting the geometric nodes of the airfoil [3]. This produced a continuous curve of vortex panels from the upper trailing edge to the lower trailing edge, aligning with a linear interpolation of the airfoil geometry. A colocation point, where the vorticities were computed to be acting, was also placed at the midpoint of each vortex panel [3]. A unitlength normal and tangent vector was computed and placed on each colocation point. A visual representation of this discretized form was presented in Figure 5.2.

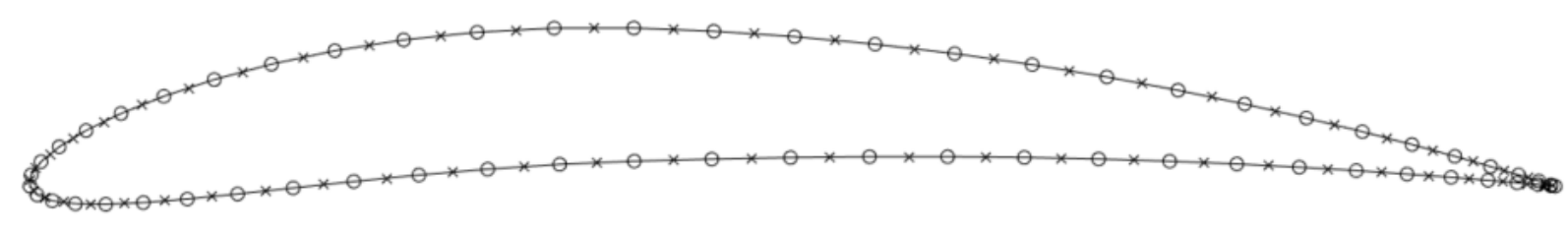

Figure 5.2: Airfoil Discretization 


\subsubsection{Mathematical Solution}

The goal of the mathematical solution was to determine the vorticity of each vortex panel, which was then used to compute the pressure coefficients and tangential velocity acting along airfoil surface. This was represented in matrix form with the following matrix relation [3]:

$$
\left[\begin{array}{cccc}
a_{11} & a_{12} & \cdots & a_{1, N+1} \\
\vdots & \vdots & \ddots & \vdots \\
a_{N 1} & a_{N 2} & \cdots & a_{N, N+1} \\
1 & 0 & \cdots & 1
\end{array}\right]\left[\begin{array}{c}
\gamma_{1} \\
\vdots \\
\gamma_{N} \\
\gamma_{N+1}
\end{array}\right]=\left[\begin{array}{c}
R H S_{1} \\
\vdots \\
R H S_{N} \\
0
\end{array}\right]
$$

Where $a$ was the aerodynamic influence coefficient, $\gamma$ was the vorticity of a panel node, and $R H S$ was the free-stream contribution. The size of the aerodynamic influence matrix was a square matrix of $N+1$, where $N$ was the number of colocation points. The size of $N+1$ resulted from the use of linear strength vortex panels over constant strength panels, as vorticities were defined at the two ends of the panels. Additionally, the final row of the matrix representation was the inclusion of the Kutta condition, which specified that the two trailing edge vorticities must equal:

$$
\gamma_{1}+\gamma_{N+1}=0
$$

The aerodynamic influence coefficients within this matrix were computed as the dot product of the selfinduced velocity of the panel, from a unit strength vortex, and the normal vector [3]. This was presented with the following equation:

$$
a_{i j}=\left\{\left(u^{b}, w^{b}\right)_{i, j-1}+\left(u^{a}, w^{a}\right)_{i, j}\right\} \cdot \hat{n}_{i}
$$

Where $u$ and $w$ were the velocity components induced by the $j$ th vorticity, and $\hat{n}_{i}$ was the unit vector normal to the $i$ th panel. The induced velocity components were calculated, in the panel coordinate system, with the following equations derived from the approach specified by Katz and Plotkin [3]:

$$
\begin{gathered}
u_{j}^{a}=-\frac{z}{2 \pi}\left(\frac{\gamma_{j}}{x_{2}-x_{1}}\right) \ln \left(\frac{r_{2}}{r_{1}}\right)+\frac{\gamma_{j}\left(x_{2}-x\right)}{2 \pi\left(x_{2}-x_{1}\right)}\left(\theta_{2}-\theta_{1}\right) \\
u_{j}^{b}=\frac{z}{2 \pi}\left(\frac{\gamma_{j+1}}{x_{2}-x_{1}}\right) \ln \left(\frac{r_{2}}{r_{1}}\right)+\frac{\gamma_{j+1}\left(x-x_{1}\right)}{2 \pi\left(x_{2}-x_{1}\right)}\left(\theta_{2}-\theta_{1}\right) \\
w_{j}^{a}=-\left(\frac{\gamma_{j}\left(x_{2}-x\right)}{2 \pi\left(x_{2}-x_{1}\right)}\right) \ln \left(\frac{r_{2}}{r_{1}}\right)-\frac{z}{2 \pi}\left(\frac{\gamma_{j}}{x_{2}-x_{1}}\right)\left[\frac{x_{2}-x_{1}}{z}+\left(\theta_{2}-\theta_{1}\right)\right] \\
w_{j}^{b}=-\left(\frac{\gamma_{j+1}\left(x-x_{1}\right)}{2 \pi\left(x_{2}-x_{1}\right)}\right) \ln \left(\frac{r_{1}}{r_{2}}\right)+\frac{z}{2 \pi}\left(\frac{\gamma_{j+1}}{x_{2}-x_{1}}\right)\left[\frac{x_{2}-x_{1}}{z}+\left(\theta_{2}-\theta_{1}\right)\right]
\end{gathered}
$$

Where the panel was defined as the line connecting points 1 and 2 , defined at locations $\left(x_{1}, z_{1}\right)$ and $\left(x_{2}, z_{2}\right)$. The vorticity acting at point 1 was set to $\gamma_{j}$, while the vorticity acting at point 2 was set to $\gamma_{j+1}$. The colocation point, for which the induced velocity was computed to be acting on, was defined as $(x, z)$ within the panel coordinate system. The distance between the colocation point and point 1 was defined as $r_{1}$, while the distance to point 2 was defined as $r_{2}$. The angles were defined as the angle between $\overrightarrow{r_{1}}$ or $\overrightarrow{r_{2}}$ and the panel tangent vector. 
To compute these values, the reference frame was required to shift into the local panel reference frame. Points were translated into the panel coordinate system with a rotation matrix [3]. Additionally, the origin of the reference frame was placed at point 1 of the panel. The computation to determine the positions within the panel reference frame was presented in the following formula [3]:

$$
\left[\begin{array}{l}
x_{p} \\
z_{p}
\end{array}\right]=\left[\begin{array}{cc}
\cos \left(\alpha_{i}\right) & \sin \left(\alpha_{i}\right) \\
-\sin \left(\alpha_{i}\right) & \cos \left(\alpha_{i}\right)
\end{array}\right]\left[\begin{array}{c}
x-x_{0} \\
z-z_{0}
\end{array}\right]
$$

Where the values $\left(x_{p}, z_{p}\right)$ were within the panel reference frame, the values $(x, z)$ were within the global reference frame, the values $\left(x_{0}, z_{0}\right)$ specified the position of the panel origin in the global coordinate system, and $\alpha_{i}$ was the incidence angle of the panel. Once the velocities were computed, another relation was required to rotate them from the panel reference into the global reference [3]. This was presented below:

$$
\left[\begin{array}{l}
u \\
w
\end{array}\right]=\left[\begin{array}{cc}
\cos \left(-\alpha_{i}\right) & \sin \left(-\alpha_{i}\right) \\
-\sin \left(-\alpha_{i}\right) & \cos \left(-\alpha_{i}\right)
\end{array}\right]\left[\begin{array}{c}
u_{p} \\
w_{p}
\end{array}\right]
$$

Where, the values $\left(u_{p}, w_{p}\right)$ were the velocity components in the panel reference frame, and the values $(u, w)$ were the velocity components in the global reference frame. The remaining component for the solution was the free-stream contribution, which was computed with the following relation [3]:

$$
R H S_{i}=\vec{Q}_{\infty} \cdot \hat{n}_{i}
$$

Where $\vec{Q}_{\infty}$ was the free-stream air velocity vector. The vorticity of each panel node was then computed, in matrix form, with the following relationship:

$$
[\gamma]=[A]^{-1}[R H S]
$$

Where $[\gamma]$ was the vorticity vector, $[A]$ was the aerodynamic influence coefficient matrix, and $[R H S]$ was the free-stream contribution vector.

To compute the induced velocity at the various colocation points, and by extension the tangential velocity and pressure coefficients, an additional matrix computation was required. This was presented in the following formula [3]:

$$
\left[\begin{array}{c}
Q_{\text {ind }, 1} \\
\vdots \\
Q_{\text {ind,N }} \\
0
\end{array}\right]=\left[\begin{array}{cccc}
b_{11} & b_{12} & \cdots & b_{1, N+1} \\
\vdots & \vdots & \ddots & \vdots \\
b_{N 1} & b_{N 2} & \cdots & b_{N, N+1} \\
0 & 0 & \cdots & 0
\end{array}\right]\left[\begin{array}{c}
\gamma_{1} \\
\vdots \\
\gamma_{N} \\
\gamma_{N+1}
\end{array}\right]
$$

Where $Q_{\text {ind }}$ was the induced velocity vector, and $b$ was the tangential aerodynamic influence coefficient. The values for the tangential coefficients were computed with the following equation [3]:

$$
b_{i j}=\left\{\left(u^{b}, w^{b}\right)_{i, j-1}+\left(u^{a}, w^{a}\right)_{i, j}\right\} \cdot \hat{t}_{i}
$$

Where $\hat{t}_{i}$ was the unit vector tangent to the panel. 


\subsubsection{Analysis Outputs}

With the vorticities and induced velocity distribution along the airfoil surface computed, the tangential velocity and pressure coefficients could be determined. The tangential velocity was taken as the sum of the induced and free-stream velocity contributions, both acting tangential to the surface. This was represented with the following equation [3]:

$$
Q_{t, j}=Q_{i n d, j}+\vec{Q}_{\infty} \cdot \hat{t}_{j}
$$

Where $Q_{t, j}$ was the tangential velocity and $\vec{Q}_{\infty}$ was the free-stream velocity vector. Utilizing the tangential velocity, the pressure coefficient acting on each panel was computed with the following relation [3]:

$$
C_{P, j}=1-\left(\frac{Q_{t, j}}{\left|\vec{Q}_{\infty}\right|}\right)^{2}
$$

Where $C_{P, j}$ was the pressure coefficient.

An additional capability, required for the effective angle of attack computation, was the calculation of the zero angle-of-attack lift coefficient for the tested airfoil. This was calculated through a summation of the pressure effects, presented in the following equation [3]:

$$
C_{l}=\sum C_{P, j} \Delta c_{j} \cos \left(\alpha_{j}\right)
$$

Where $C_{l}$ was the lift coefficient, $\Delta c_{j}$ was the distance between the $j$ th and $(j+1)$ th colocation point, and $\alpha_{i}$ was the incident angle of the panel.

\subsection{Viscous Airfoil Analysis}

The viscous analysis of the airfoil was determined using the analysis scheme presented by Fujiwara et al [4]. This required the tangential velocity distribution along the upper and lower airfoils surfaces, and determined the corresponding sectional drag and boundary layer displacement thickness. The chosen approach was to implement a direct boundary layer scheme, due to its simplicity over the simultaneous solution method. While the simultaneous method would be capable of predicting low-Reynolds number performance accurately, this capability was considered to be unnecessary as the VLM mandated large Reynolds number cases [4]. As such, the direct method was selected, wherein the viscous solution was computed after the inviscid solution and was used to modify the geometry input to the inviscid calculator.

This analysis was computed twice for a given airfoil, once for each airfoil surface. As a precursor to execution, the airfoil was divided at its stagnation point, or whichever node presented the lowest tangential speed. 


\subsubsection{Laminar Boundary Layer}

The laminar boundary layer solution was a compressible implementation of Curle's method [8], which was an adjustment on Thwaites' method [4], with empirical relations used to compute the boundary layer characteristics at each node using the previous node properties. For each node, the momentum thickness was calculated first using the tangential edge velocity computed by the inviscid airfoil solver. This was presented in the following relation:

$$
\theta^{2} u_{e}^{6}=0.441 \frac{\mu}{\rho} \int_{0}^{s} u_{e}^{5} d s
$$

Where $\theta$ was the momentum thickness of the boundary layer, $u_{e}$ was the inviscid edge velocity set equal to the tangential speed computed by the inviscid solution, $\mu$ was the viscosity of air, $\rho$ was the density of air, and $s$ was the distance along the airfoil surface curve from the stagnation point. Converting this equation into a numerical iteration problem, it was computed in discrete form using the following equation:

$$
\theta=\sqrt{\frac{0.441 \mu}{\rho u_{e, i}^{6}} \sum_{j=1}^{i} u_{e, j}^{5} \Delta s_{j}}
$$

Where $i$ was the index of the panel being solved for. For this panel, a local Reynolds number per unit length was also defined, as presented with the following definition [4]:

$$
R e_{l}=\frac{\rho u_{e}}{\mu}
$$

This value was subsequently used to compute the momentum thickness Reynolds number with the following relation:

$$
R e_{\theta}=R e_{l} \theta
$$

Where $R e_{\theta}$ was the momentum thickness Reynolds number. This value was used to determine if the flow has transitioned to turbulence using the condition presented in Section 5.4.2.

Following with Thwaites' approach, the dimensionless pressure-gradient parameter was computed through the following approximation [4]:

$$
\lambda=\frac{\theta^{2} \rho}{\mu} \frac{\Delta u_{e}}{\Delta s}
$$

Where $\lambda$ was the pressure-gradient parameter, and $\Delta u_{e} / \Delta s$ was the velocity gradient of the panel. This parameter was input to Twaites' empirical relations for the shape factor [4]:

$$
H=\left\{\begin{array}{cc}
3.9155 & , \lambda \leq-0.1 \\
2.088+\frac{0.0731}{\lambda+0.14} & ,-0.1<\lambda \leq 0.0 \\
2.61-3.75 \lambda+5.24 \lambda^{2} & , 0.0<\lambda \leq 0.1 \\
2.2874 & , \lambda>0.1
\end{array}\right.
$$

Where $H$ was the shape factor. 
An additional parameter, the $l$ parameter was also computed empirically using relations developed by Thwaites, as presented below [4]:

$$
l=\left\{\begin{array}{cc}
-0.1773 & , \lambda \leq-0.1 \\
0.22+1.402 \lambda+\frac{0.018 \lambda}{\lambda+0.107}, & -0.1<\lambda \leq 0.0 \\
0.22+1.57 \lambda-1.8 \lambda^{2} & , 0.0<\lambda \leq 0.1 \\
0.3590 & , \lambda>0.1
\end{array}\right.
$$

With the two parameters determined, the boundary layer displacement thickness and friction coefficient were computed for each panel. This was done with the following two relations [4]:

$$
\begin{gathered}
\delta^{*}=H \theta \\
C_{f}=\frac{2 l}{R e_{\theta}}\left(1+0.2 M^{2}\right)
\end{gathered}
$$

Where $\delta^{*}$ was the displacement thickness of the boundary layer, $C_{f}$ was the compressible friction coefficient for the panel, and $M$ was the Mach number of the inviscid flow for the panel. The boundary layer properties were defined by equations (5.23) and (5.24) until the transition condition was met, and the turbulent analysis was used in its stead.

\subsubsection{Transition Condition}

The transition condition, following the approach of Fujiwara et al, was achieved through implementing Michel's transition criterion [4]. This first required the computation of the arc-length Reynolds number, as presented in the following definition:

$$
R e_{S}=R e_{l} S
$$

Where $R e_{s}$ was the arc-length Reynolds number, and $s$ was the distance along the airfoil surface curve from the stagnation point. The transition condition was subsequently computed with the following definition [4]:

$$
R e_{\theta, \text { transition }}=1.174\left(1+\frac{22400}{R e_{s}}\right) R e_{s}^{0.46}
$$

Where $R e_{\theta, t r a n s i t i o n}$ was the transition momentum thickness Reynolds number. This condition was computed for each panel within the laminar region, and once the momentum thickness Reynolds number was found to exceed this parameter, the flow was considered to have transitioned to turbulent flow. For this panel and all subsequent panels to the trailing edge, the turbulent boundary layer analysis was used.

\subsubsection{Turbulent Boundary Layer}

Once the flow exceeded the transition condition, the turbulent boundary layer model was used. The implementation involved using the boundary layer properties of a given panel to compute the characteristics at the succeeding panel. The first step of this process was computing the boundary layer shape factor with the following relation, developed by Stanford University through modifying the approach developed by Cebeci and Schlichting [9]:

$$
H_{1}=3.0445+\frac{0.8702}{(H-1.1)^{1.2721}}
$$

Where $H_{1}$ was the boundary layer shape factor, and $H$ was the shape factor. 
Another mathematical quantity required was termed the $U T H$ factor and was computed with the following relation. This was required to use an empirical differential relationship presented by Fujiwara et al [4].

$$
U T H=u_{e} \theta H_{1}
$$

Where UTH was the needed factor. This was required to compute the properties of the succeeding panel according to the following relation [4] which defined the change in this quantity with respect to the airfoil arc.

$$
\frac{d}{d s}(U T H)=\frac{0.0306 u_{e}}{\left(H_{1}-3.0\right)^{0.6169}}
$$

Using equation (5.29), an iterative scheme was produced to predict the value of the UTH factor for the succeeding panel, as presented in the following formula where Euler's method was employed:

$$
U T H_{i+1}=U T H_{i}+\frac{d}{d s}(U T H) \cdot \Delta s
$$

Where $\Delta s$ was the length of the panel, equal to the arc length step. Computing the succeeding panel momentum thickness value required the use of the von Karmen's compressible integral momentum equation, reordered in the following relation [4]:

$$
\frac{d \theta}{d s}=\frac{C_{f}}{2}-\frac{\theta}{u_{e}}\left(2+H-M^{2}\right) \frac{\Delta u_{e}}{\Delta s}
$$

This equation required known properties of the panel, but also required the friction coefficient. This was computed with the empirical relationship presented below [4]:

$$
C_{f}=\frac{0.246}{10^{0.678 H} R_{\theta}^{0.268}}\left(1+0.2 M^{2}\right)
$$

Where $R e_{\theta}$ was the momentum thickness Reynolds number, and $M$ was the Mach number of the flow above the panel. With the displacement thickness gradient defined, Euler's method was again used to compute the momentum thickness of the succeeding panel with the following iterative definition:

$$
\theta_{i+1}+\theta_{i}+\frac{d \theta}{d s} \Delta s
$$

Using equations (5.30) and (5.33), the properties of the succeeding panels were able to be computed. First, the boundary layer shape factor was computed from the momentum thickness, the inviscid tangential velocity, and the UTH factor as presented below:

$$
H_{1}=\frac{U T H}{\theta u_{e}}
$$

The shape factor corresponding to this value was subsequently computed with the following empirical definition [4]:

$$
H=\left\{\begin{array}{cc}
3.0 & , H_{1}<3.33 \\
0.6778+1.1536\left(H_{1}-3.3\right)^{-0.326} & , 3.3 \leq H_{1} \leq 5.3 \\
1.1+0.86\left(H_{1}-3.3\right)^{-0.777} & H_{1}>5.3
\end{array}\right.
$$

Finally, the boundary layer displacement thickness was computed with equation (5.23), restated below:

$$
\delta^{*}=H \theta
$$


With the displacement thickness and friction coefficients determined, the relevant quantities of the boundary layer were able to be modelled. Additionally, following the recommendations of Fujiwara et al, once the solution was completed, the boundary layer thickness for the last $5 \%$ of the chord was overridden to have a constant thickness, correcting for the strong adverse pressure gradients produced by the trailing edge Kutta condition [4].

\subsection{Inviscid-Viscous Coupling}

Coupling of the inviscid and viscous airfoil calculators was achieved through an iterative scheme. First, the inviscid solution was computed using the defined airfoil geometry. The viscous analysis was subsequently computed using the tangential velocity distribution predicted by the inviscid analysis.

The viscous analysis generated a boundary layer displacement thickness as one of its outputs. This was used to adjust the airfoil geometry provided to the inviscid calculator, so that the inviscid analysis was conducted about the boundary layer rather than the airfoil geometry itself. This defined the iterative scheme, which was exited once the boundary layer thickness at the middle of the airfoil was no longer changing significantly.

A sample result was provided in Figure 5.3, showing a magnified view of an airfoil and boundary layer. The boundary layer displacement thickness was visibly thin, and only adjusted the geometry of the airfoil slightly. Nonetheless, the difference in predicted skin friction was significant between the first and last iteration, justifying the need for the iterative procedure.

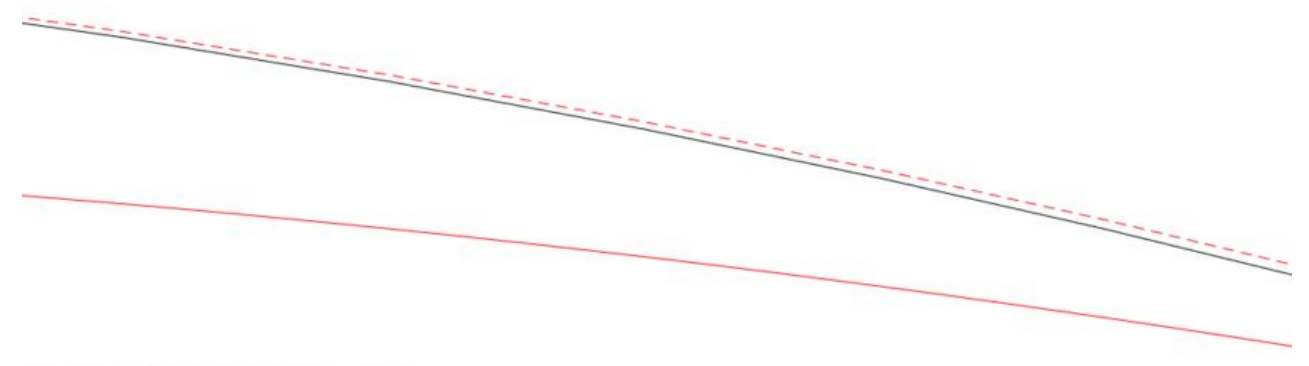

Airfoil Outline

Boundary Layer Displacement

Figure 5.3: Viscous Airfoil Analysis Output

\subsection{Parasitic Drag Computation}

The parasitic drag acting on each section was computed from the friction coefficients output by the boundary layer analysis. This was defined with the following relation:

$$
d_{p}=\frac{1}{2} \rho \sum Q_{t, i}^{2} C_{f, i} \Delta s_{i}
$$

Where $d_{p}$ was the parasitic drag per unit length acting on the section, and $\Delta s$ was the length of each panel comprising the airfoil. 
The overall parasitic drag force acting on the section was computed by multiplying the sectional drag with the section width, as presented in the following equation:

$$
\Delta D_{p, i}=d_{p, i} \Delta y_{i}
$$

Where $\Delta D_{p}$ was the parasitic drag force acting on the section, and $\Delta y$ was the spanwise length of the section. The total parasitic drag acting on the wing was taken as the summation of the drag force acting on each constituent section:

$$
D_{p}=\sum \Delta D_{p, i}
$$

Where $D_{p}$ was the total parasitic drag acting on the wing.

\section{Structural Analysis}

Structural modelling was utilized to predict how the wing would deform under the loading conditions applied to it. This was done through an implementation of classical beam theory, which predicted the bending deflection and twist angle of each section along the wing.

\subsection{Wing Box Bending Model}

To facilitate computation of the wing box bending properties, the structure was modelled as a series of area concentrations. Through analysing the wing box in this form, an accurate approximation was possible for the moments of inertia and centroid, provided a sufficient resolution was utilized. By extension, this enabled the computation of the principal axes which were required to accurately predict the response of asymmetric cross sections. A visual representation of this discretization was presented in Figure 6.1, depicting the skin, spars, and stringers as a series of area concentration points.

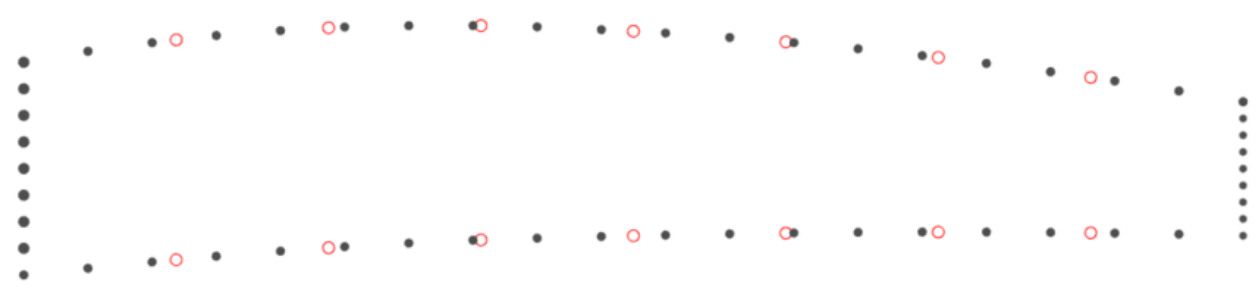

Figure 6.1: Wing Box as Area Concentrations 
This numerical approximation scheme allowed for the following approximations to be used for the centroid computations [5]:

$$
\begin{aligned}
& x_{c}=\frac{\sum x_{i} A_{i}}{\sum A_{i}} \\
& z_{c}=\frac{\sum z_{i} A_{i}}{\sum A_{i}}
\end{aligned}
$$

Where $\left(x_{c}, z_{c}\right)$ was the location of the centroid, $\left(x_{i}, z_{i}\right)$ was the location of each area concentration, and $A_{i}$ was the area concentrated at each point. The moments and product of inertia were similarly computed according to the following numerical definitions [5]:

$$
\begin{gathered}
I_{x x}=\sum z_{i}^{2} A_{i} \\
I_{z z}=\sum x_{i}^{2} A_{i} \\
I_{z x}=\sum x_{i} z_{i} A_{i}
\end{gathered}
$$

Where $I_{x x}$ and $I_{z z}$ were the area moments of inertia, and $I_{z x}$ was the product of inertia. With these computations, the moments and product of inertia were computed for the centroidal reference frame which was defined as having the origin placed at the centroid with the axes parallel to the global $x$ - and $z$-axes.

For the computation of the bending response, the principal axes were required. This was computed according to the definition provided by Hibbeler [5]:

$$
\theta_{p}=\frac{1}{2} \arctan \left(-\frac{I_{z x}}{0.5\left(I_{x x}-I_{z z}\right)}\right)
$$

Where $\theta_{p}$ was the angle between the principal axis and the global axis. Note that MATLAB's atand() function was used here to ensure the angle was bounded by $[-90,90]$. A visual representation of the principal axes relative to the centroidal axes was provided in Figure 6.2.

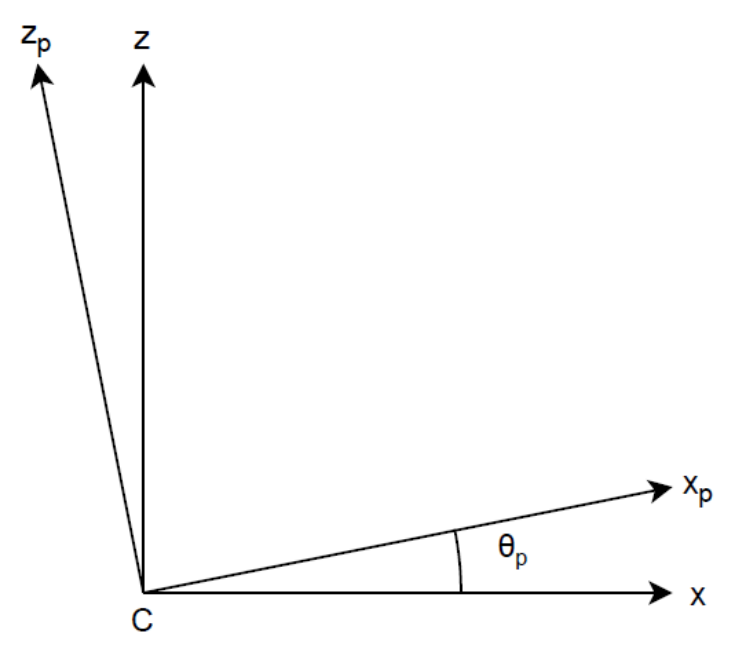

Figure 6.2: Principal Axes 
Within the principal reference frame, defined by the principal axes, the global-reference moments and product of inertia were computed according to the following equations [5]:

$$
\begin{gathered}
I_{x^{\prime} x^{\prime}}=\frac{I_{x x}+I_{z z}}{2}+\frac{I_{x x}-I_{z z}}{2} \cos \left(2 \theta_{p}\right)-I_{z x} \sin \left(2 \theta_{p}\right) \\
I_{z^{\prime} z^{\prime}}=\frac{I_{x x}+I_{z z}}{2}+\frac{I_{x x}-I_{z z}}{2} \cos \left(2 \theta_{p}\right)+I_{z x} \sin \left(2 \theta_{p}\right) \\
I_{z^{\prime} x^{\prime}}=0
\end{gathered}
$$

Where $I_{x^{\prime} x^{\prime}}$ and $I_{z^{\prime} z^{\prime}}$ were the moments of inertia within the principal reference frame, and $I_{z^{\prime} x^{\prime}}$ was the product of inertia within the principal reference frame, set to zero by definition.

Converting the coordinates of points into and out of the principal reference frame was done according to the following matrix definitions:

$$
\begin{aligned}
& {\left[\begin{array}{l}
x^{\prime} \\
z^{\prime}
\end{array}\right]=\left[\begin{array}{cc}
\cos \theta_{p} & \sin \theta_{p} \\
-\sin \theta_{p} & \cos \theta_{p}
\end{array}\right]\left[\begin{array}{l}
x \\
z
\end{array}\right]} \\
& {\left[\begin{array}{l}
x \\
z
\end{array}\right]=\left[\begin{array}{cc}
\cos \theta_{p} & -\sin \theta_{p} \\
\sin \theta_{p} & \cos \theta_{p}
\end{array}\right]\left[\begin{array}{l}
x^{\prime} \\
z^{\prime}
\end{array}\right]}
\end{aligned}
$$

Where $\left(x^{\prime}, z^{\prime}\right)$ was the coordinates of a given point in the principal reference frame, and $(x, z)$ were the coordinates of the same point within the centroidal reference frame.

\subsection{Wing Box Torsion Model}

To compute the torsion response of the wing, the shear centre location was required. This was computed by applying an arbitrary unit-magnitude shear force in the $x^{\prime}$-direction to locate the $z^{\prime}$-coordinate of the shear center. Following this, a unit magnitude force was applied in the $z^{\prime}$-direction and was used to locate the $x^{\prime}$-coordinate of the shear center.

Locating the shear center first required the shear flow within the structure to be computed. This was defined with the following equation [10]:

$$
q_{s}=-\left(\frac{S_{x} I_{x x}-S_{z} I_{z x}}{I_{x x} I_{z z}-I_{z x}^{2}}\right) \int_{0}^{s} t x d s-\left(\frac{S_{z} I_{z z}-S_{x} I_{z x}}{I_{x x} I_{z z}-I_{z x}^{2}}\right) \int_{0}^{s} t y d s+q_{s, 0}
$$

Where $q_{s}$ was the shear flow, $q_{s, 0}$ was the constant shear flow, $S_{x}$ and $S_{z}$ were the applied shear forces, and $s$ was the distance along the curve of the wing box surface. The summation of the two integral terms and their coefficients was termed the "basic shear flow" [10], and was given the identifier $q_{b}$.

Numerically approximating the basic shear flow, and computing its value within the principal reference frame, and thereby setting the product of inertia to zero, the following formula was used to compute this parameter for the analyser:

$$
q_{b, i}=-\frac{s_{x}}{I_{z^{\prime} z^{\prime}}} \sum_{j=i}^{i}\left(t_{i} x_{j}^{\prime} \Delta s_{j}\right)-\frac{s_{z}}{I_{x^{\prime} x^{\prime}}} \sum_{j=0}^{i}\left(t_{j} z_{j}^{\prime} \Delta s_{j}\right)
$$

Where $q_{b, i}$ was the basic shear flow value at a wing box node, $t_{i}$ was the thickness of the skin or spar at that node, $\left(x_{j}^{\prime}, z_{j}^{\prime}\right)$ was the coordinates of that node within the principal reference frame, and $\Delta s_{j}$ was the distance between the node and the previous node. The selection of the initial node, and thereby the first index, was arbitrary and did not affect the solution to the overall shear flow. 
The constant shear flow was also computed using a numerical approximation, defined by the following formula [10]:

$$
q_{s, 0}=-\frac{\sum q_{b, i} \Delta s_{i} / t_{i}}{\sum \Delta s_{i} / t_{i}}
$$

With the shear flow numerically approximated along the aircraft surface, the following definition was used as a basis for computing the location of the shear centre [10]:

$$
S_{x} \eta-S_{z} \xi=\oint p q_{b} d s+2 A_{\text {mean }} q_{s, 0}
$$

Where $\eta$ was the $z$-coordinate of the shear center, $\xi$ was the $x$-coordinate of the shear center, $p$ was the lever arm of the shear flow acting on the origin of the principal reference frame, and $A_{\text {mean }}$ was the mean 2D area encased by the wing box. This was numerically approximated into the following form:

$$
S_{x} \eta-S_{z} \xi=\sum p_{i} q_{b, i} \Delta s_{i}+2 A_{\text {mean }} q_{s, 0}
$$

Additionally, with a derivation provided in Appendix B, the shear arm was computed with the following formula:

$$
p_{i}=\left|\frac{z_{i}-\frac{\Delta z}{\Delta x} x_{i}}{1-\left(\frac{\Delta z}{\Delta x}\right)^{2}}\right| \sqrt{\left(\frac{\Delta z}{\Delta x}\right)^{2}+1}
$$

Where $p_{i}$ was the lever arm corresponding to the wing box node, $\Delta z / \Delta x$ was the slope of the wing box at the node, and $\left(x_{i}, z_{i}\right)$ was the coordinates of the node. Equation (6.11) was used twice, once with the $x^{\prime}-$ direction force and a second time with $z^{\prime}$-direction force. For each, the force in the other direction was set to zero and the coordinates were solved through algebraic manipulation. The coordinates were then rotated back into the centroidal reference frame with equation (6.6b).

\subsection{Wing Box Scaling Factors}

For computational expediency, the properties of the wing box were computed to correspond to a unit-length airfoil cross section. Its various characteristics were then multiplied by scaling factors to produce the properties of the wing box at a given section. These were presented in Table 6.1, where properties were scaled as a function of chord length $c$.

Table 6.1: Wing Box Scaling Factors

\begin{tabular}{|c|c|}
\hline Parameter & Scaling Factor \\
\hline Length $(\Delta s)$ & $c$ \\
\hline Skin/Spar Thickness & (unchanged) \\
\hline Coordinates $(x, z)$ & $c$ \\
\hline Area Concentration $\left(A_{i}\right)$ & $c$ \\
\hline Mean Area $\left(A_{\text {mean }}\right)$ & $c^{2}$ \\
\hline Moment of Inertia $\left(I_{x x}, I_{z z}\right)$ & $c^{3}$ \\
\hline Product of Inertia $\left(I_{z x}\right)$ & $c^{3}$ \\
\hline Principal Angle $\left(\theta_{p}\right)$ & (unchanged) \\
\hline
\end{tabular}




\subsection{Wing Loading}

The loading applied to the wing was computed through analysing the lift forces generated by the VLM on each section, producing a load distribution along the wingspan. As with the viscous aerodynamic analyzer, the sections were divided by the panel boundaries which were parallel to the free stream.

\subsubsection{Torsional Loads}

Computing first the torsional loading, the torque acting on each section was computed with the following formula:

$$
T_{s, j}=\sum \Delta L_{i}\left(x_{s c}-x_{i}\right)
$$

Where $T_{s, j}$ was the torque acting on the section, $\Delta L_{i}$ was the lift force acting on teach panel within the section, $x_{i}$ was the colocation point $x$-coordinate where the force was acting, and $x_{s c}$ was the shear centre $x$-coordinate.

To compute the torsion distribution along the wingspan, the torque acting on the root section was first set as the negative total torque acting on the wing, as defined in the following equation [5]:

$$
T\left(y_{1}\right)=-\sum T_{s, j}
$$

Where $T\left(y_{1}\right)$ was the value of the torque distribution function at the root. The values for the torque distribution function were then computed iteratively according to the following formula:

$$
T\left(y_{j}\right)=T\left(y_{j-1}\right)+T_{s, j}
$$

Where $y$ was the spanwise coordinate.

\subsubsection{Bending Loads}

To compute the bending loads, the shear force and bending moment functions were defined along the span of the wing. First, the shear load distribution was computed, with the shear load acting on the root section set to equal the negative total lift force acting on the wing [5]:

$$
V\left(y_{1}\right)=-L
$$

Where $V\left(y_{1}\right)$ was the value of the shear load function at the root. The subsequent values for the shear load function were defined iteratively as presented below:

$$
V\left(y_{j}\right)=V\left(y_{j-1}\right)+\sum \Delta L_{i j}
$$

Where $\sum \Delta L_{i j}$ was the total lift force acting on the section.

The moment distribution was computed in a similar iterative fashion. The bending moment at the wing tip was first set to zero [5]:

$$
M\left(y_{n}\right)=0
$$

Where $M\left(y_{n}\right)$ was the value of the moment function at the wing tip. The remaining values for the function were computed iteratively from the tip to the root with the following definition [5]:

$$
M\left(y_{j}\right)=M\left(y_{j+1}\right)+\frac{1}{2} \Delta y_{j}\left(\sum \Delta L_{i j}\right)
$$

Where $\Delta y_{j}$ was spanwise panel width, and $\sum \Delta L_{i j}$ was the lift force acting on the section. 


\subsection{Wing Deformation}

Deformation of the wing was computed from the twist and deflection distribution along the span. For this analysis, first the twist angle distribution along the wing was computed. Following this, the deflection angle and vertical deflections were found, using the twist angle to adjust the point tested in determining the radius of curvature.

\subsubsection{Twist Angle Prediction}

The twist angle at each point along the wingspan was calculated through the following approximation provided by Hibbeler [5]. The twist angle at the root was set to zero degrees, while each successive angle was computed with the torsion distribution function.

$$
\phi_{j+1}=\phi_{j}-\frac{T\left(y_{j}\right) \Delta y_{j}}{4 A_{\text {mean }}^{2} G} \sum \frac{\Delta s_{i}}{t_{i}}
$$

Where $\phi$ was the twist angle and $\Delta y_{j}$ was the spanwise panel width. The quantity $\sum \frac{\Delta s_{i}}{t_{i}}$ was computed along the wing box surface.

\subsubsection{Deflection Prediction}

Prediction of the deflection required the radius of curvature for the wing box to be determined at each spanwise section. To compensate for the twist of the wing and ensure that the computed deflection was in the vertical direction, the point probed to compute this value was rotated by the twist angle.

Prior to this computation, however, the moment vector components acting on the structure were required. Resulting from the lift force distribution, this vector was parallel to the global $x$-axis while the components acting along the principal axes was needed, and further accounting for the rotation caused by twist was necessary.

The deconstruction of the moment vector was presented in Figure 6.3. In this figure the axes shown were centered on the wing box centroid. The $x$-axis was set parallel to the global $x$-axis, while the $x_{c}$-axis was set parallel to the untwisted $x$-axis of the wing box. Finally, the $x_{p}$-and $z_{p}$-axes were the principal axes of the wing box structure.

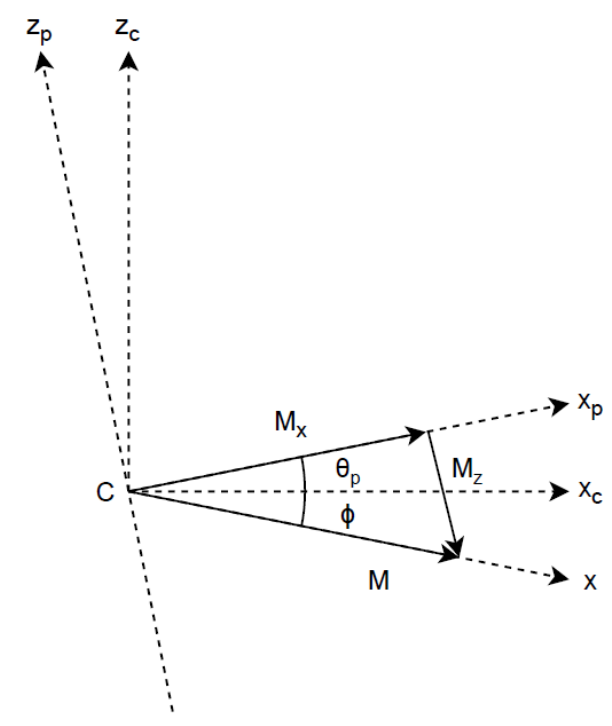

Figure 6.3: Moment Vector Components 
Geometrically derived from Figure 6.3, the bending moment vector acting on the wing box was separated into its constituent principal-axis components with the following to equations:

$$
\begin{aligned}
& M_{x}=M \cos \left(-\theta_{p}-\phi\right) \\
& M_{z}=M \sin \left(-\theta_{p}-\phi\right)
\end{aligned}
$$

Where $M_{x}$ was the moment vector component about the principal $x$-axis, $M_{z}$ was the moment vector component about the $z$-axis, and $M$ was the total bending moment acting on the cross section.

Having determined the bending moment components acting on the structure, the lateral strain acting on any arbitrary point within the wing box structure was able to be computed with the following formula [5]:

$$
\varepsilon_{y}=\frac{M_{z} x^{\prime}}{E I_{z^{\prime} z^{\prime}}}-\frac{M_{x} z^{\prime}}{E I_{x^{\prime} x^{\prime}}}
$$

Where $\varepsilon_{y}$ was the lateral strain, $\left(x^{\prime}, z^{\prime}\right)$ was the coordinates of an arbitrary point defined in the principal reference frame, and $E$ was the elastic modulus of the material. To compute the lateral strain value used for the deflection angle computation, an arbitrary point near the top of the wing box was probed. To align the radius of curvature and ensure that the computed deflection was vertical, the probed point was rotated from the centroidal axis by the twist angle as presented in Figure 6.4.

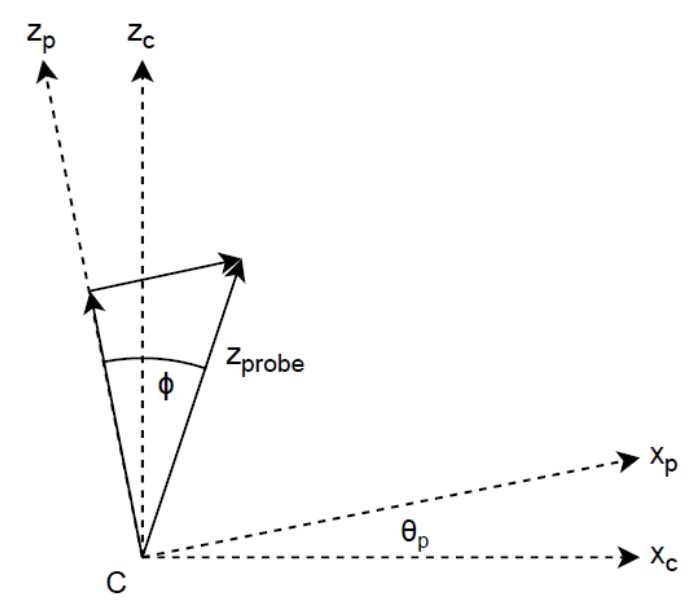

Figure 6.4: Probe Point Selection

Deriving from the geometry presented in Figure 6.4, the probed point defined within the principal reference frame was computed with the following equations:

$$
\begin{aligned}
x^{\prime} & =z_{\text {probe }} \sin \left(\theta_{p}+\phi\right) \\
z^{\prime} & =z_{\text {probe }} \cos \left(\theta_{p}+\phi\right)
\end{aligned}
$$

Where $\left(x^{\prime}, z^{\prime}\right)$ was the probed point coordinates defined in the principal reference frame, and $z_{\text {probe }}$ was the $z$-axis component of the point probed for the strain. 
With the lateral strain value computed, the deflection angle slope was able to be approximated with the following equation [5]:

$$
\frac{d \theta}{d y}=-\frac{\varepsilon_{y}}{z_{\text {probe }}}
$$

Where $\theta$ was the deflection angle, and $d \theta / d y$ was the deflection angle slope. Employing Euler's method alongside an initial deflection angle of zero at the root, the successive deflection angles along the wingspan were computed with the following formula:

$$
\theta_{j+1}=\theta_{j}+\frac{d \theta}{d y} \Delta y_{j}
$$

Where $\Delta y_{j}$ was the spanwise section length. Conducting this computation along the entire wingspan resulted in the deflection angle being determined for each section of the wing.

Finally, with the deflection angle at each location along the wing computed, the vertical deflection was determined through a similar iterative scheme. This was presented in the following formula, where the deflection at the wing root was set to zero.

$$
\Delta z_{j+1}=\Delta z_{j}+\tan \theta_{j} \Delta y_{j}
$$

Where $\Delta z$ was the vertical deflection of the wing section.

\subsubsection{Mesh Deformation}

To deform the VLM mesh using the predetermined twist and deflection distribution, the wing was again divided into spanwise sections, aligning with the free-stream-parallel panel boundaries. Each point defining the panel geometry was rotated and deflected according to the following equation:

$$
\begin{gathered}
x_{d}=\sqrt{x^{2}-z^{2}} \cos \left(\arctan \left(\frac{z}{x}\right)+\phi_{j}\right) \\
z_{d}=\sqrt{x^{2}-z^{2}} \sin \left(\arctan \left(\frac{z}{x}\right)+\phi_{j}\right)+\Delta z_{j}
\end{gathered}
$$

Where $\left(x_{d}, z_{d}\right)$ was the location of the panel point after it was deformed, and $(x, z)$ was the location of the panel point before the deformation. Additionally, MATLAB's arctan2() function was used to compute the angle to ensure that the point was rotated from the correct quadrant. 


\section{Sample Results}

\subsection{Tested Wing Configuration}

To demonstrate the output capabilities of the developed multidisciplinary analysis program, a generic lowsweep wing was utilized, intended to represent a typical turboprop aircraft. To further illustrate the wing twisting, a longer wing was utilized. The parameters which defined this computation were presented in Table 7.1.

Table 7.1: Sample Parameters

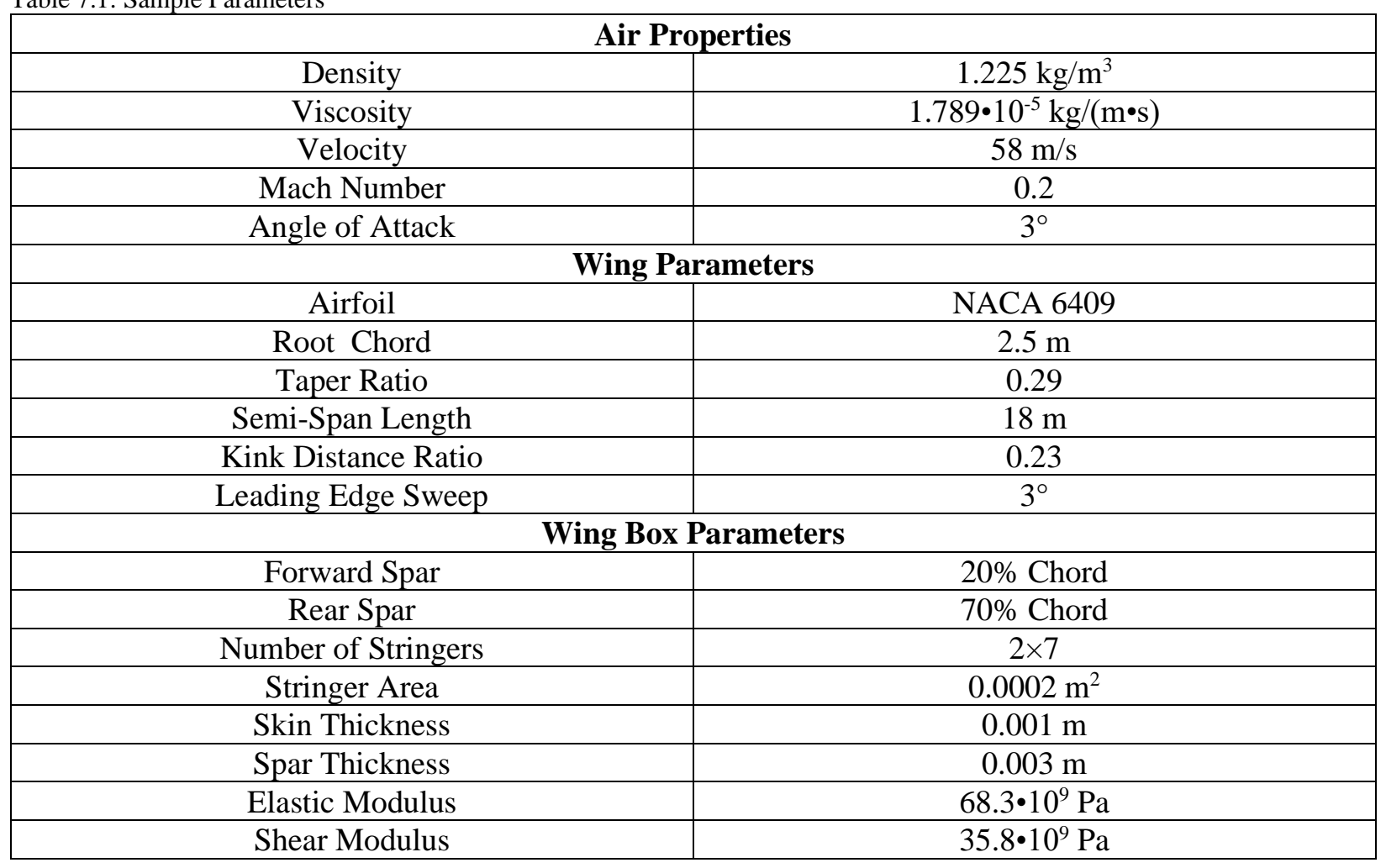

\subsection{Aerodynamic Results}

\subsubsection{Lift Distribution}

One of the core outputs of the VLM was the lift distribution along the aircraft wing. This was presented in Figure 7.1, where greater lift forces were demarked in yellow and lower forces were indicated with blue.

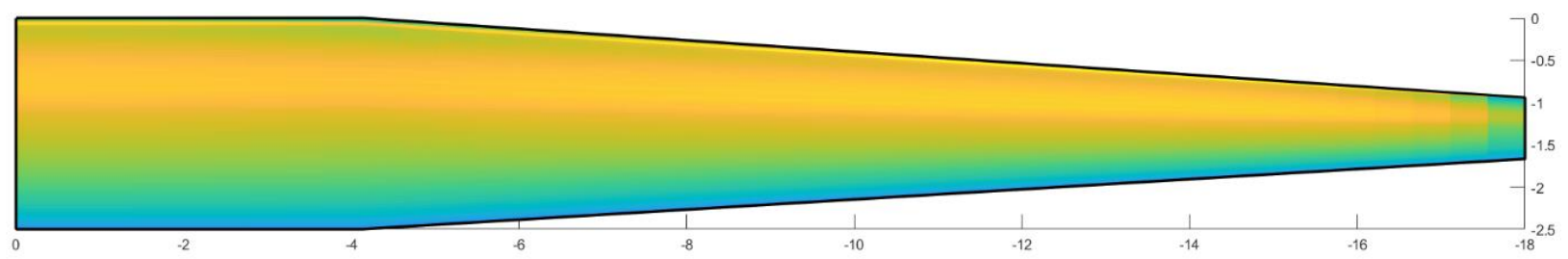

Figure 7.1: Sample Lift Distribution 


\subsubsection{Aerodynamic Forces}

Combining the outputs of both aerodynamic analyzers, the aerodynamic forces acting on the aircraft were computed and presented in Table 7.2. This included the lift and induced drag, which were computed by the VLM, and the parasitic drag output by the viscous analyzer. The overall lift to drag ratio of the wing was also presented.

Table 7.2 Sample Aerodynamic Forces

\begin{tabular}{|c|r|}
\hline Output & \multicolumn{1}{|c|}{ Value } \\
\hline Lift & $61370 \mathrm{~N}$ \\
\hline Induced Drag & $870 \mathrm{~N}$ \\
\hline Parasitic Drag & $430 \mathrm{~N}$ \\
\hline Lift-to-Drag Ratio & 47 \\
\hline
\end{tabular}

\subsection{Wing Loading Results}

\subsubsection{Bending Loads}

The loading outputs predicted by the bending solver include the shear force and bending moment distribution. The shear force corresponding to this sample case, modeled as a function of spanwise distance, was presented in Figure 7.2. The bending moment distribution which corresponded to this was presented in Figure 7.3.

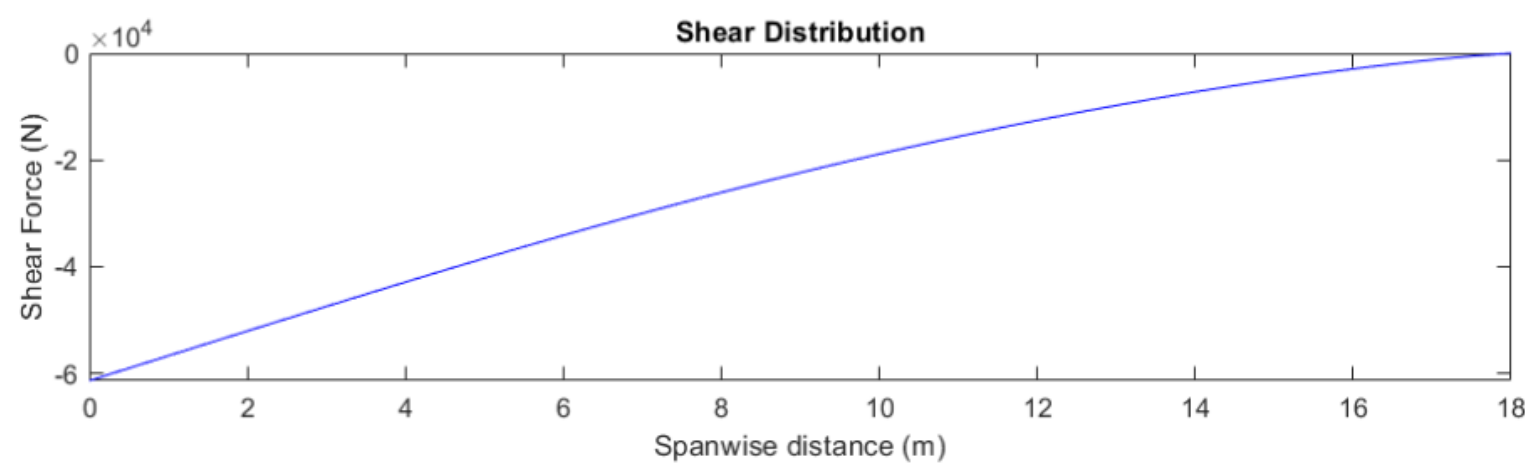

Figure 7.2: Sample Shear Distribution

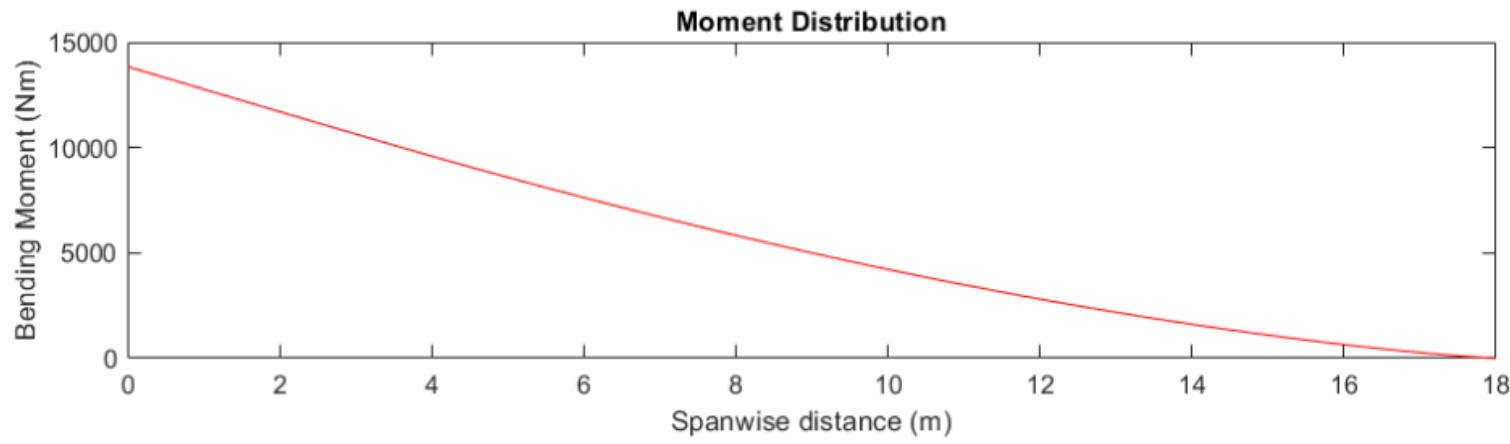

Figure 7.3: Sample Bending Moment Distribution 


\subsubsection{Torsion Loads}

The torsion load calculator was responsible for predicting the torque distribution as a function of the spanwise distance. The plot presenting this for the sample case was given in Figure 7.4.

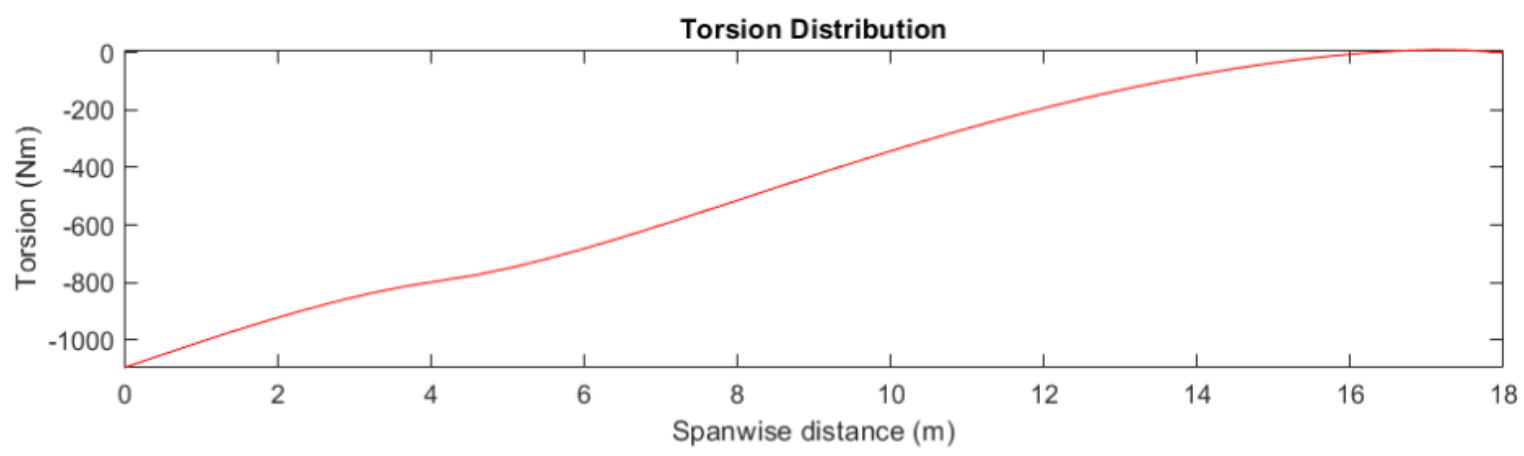

Figure 7.4: Sample Torsion Distribution

\subsubsection{Resulting Deformation}

The core functionality of the wing structural analyzer was the bending deflection and twist angle as a function of spanwise distance. The bending deflection predicted for this sample cases was presented in Figure 7.5, while the predicted twist was shown in Figure 7.6.

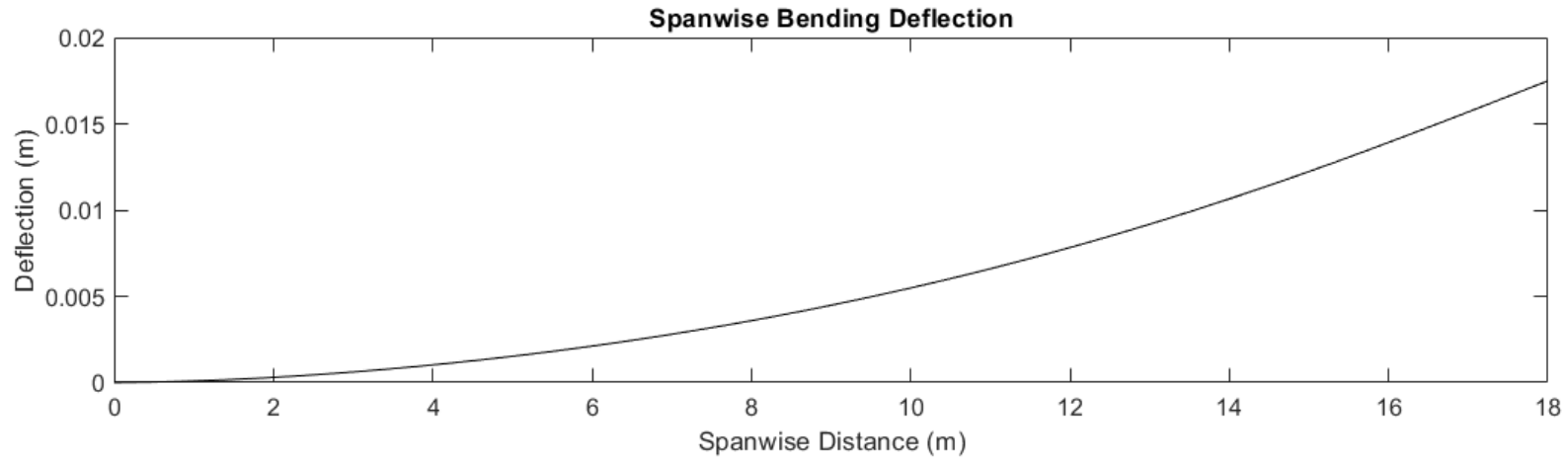

Figure 7.5: Sample Wing Deflection

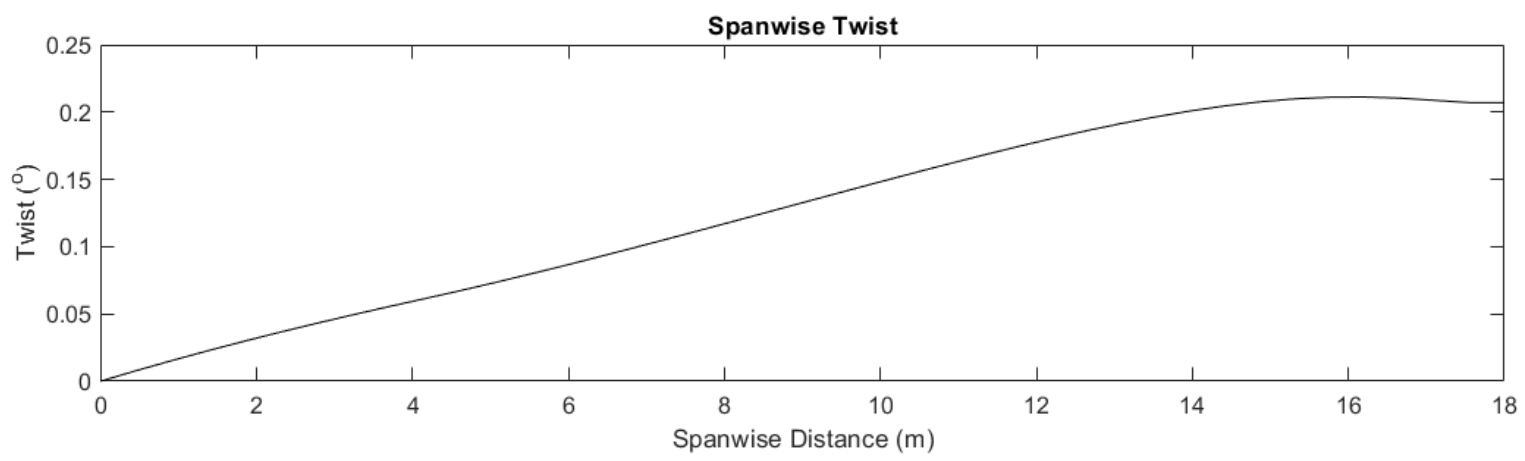

Figure 7.6: Sample Wing Twist 


\subsection{Computer Performance}

As the developed program was a numerical approximation, a trade-off existed between simulation resolution and compute time. A series of resolutions were tested, with the required iterations and time to compute presented in Table 7.3. These results were produced using a MATLAB environment given 24 parallel workers, on an AMD Ryzen-9 3950X computer system with 16 CPU cores and 32 gigabytes of RAM. During the program operation, the system was able to maintain approximately $90 \%$ CPU utilization with the developed architecture.

Table 7.3: Computational Requirements

\begin{tabular}{|r|r|r|r|}
\hline Number of $\boldsymbol{x}$-panels & Number of $\boldsymbol{y}$-panels & Iterations to Converge & \multicolumn{1}{c|}{ Time to Compute (s) } \\
\hline 3 & 7 & 10 & 1.4 \\
\hline 10 & 40 & 7 & 4.2 \\
\hline 40 & 40 & 4 & 20.8 \\
\hline 60 & 40 & 5 & 55.8 \\
\hline 60 & 80 & 5 & 213.9 \\
\hline 60 & 100 & 5 & 330.2 \\
\hline
\end{tabular}

\section{Conclusions}

An aerostructural analyzer was developed to model the deformation and aerodynamic loading of a lowsweep wing. This implementation was developed within the MATLAB environment, and included coupled aerodynamic and structural solvers. Each of these encapsulated solving functions were further subdivided into additional functions which sequentially or iteratively generated the required outputs.

The aerodynamic analysis approach used was the quasi-three-dimensional modelling approach, with a separate inviscid and viscous analyzer [2]. The inviscid effects were modelled with a vortex ring lifting surface model, implementing the method presented by Katz and Plotkin [3]. Viscous effects, specifically that of parasitic drag, were modelled with strip theory analysis using a 2D airfoil analyzer [2]. This 2D analysis was in itself subdivided, including an effective angle of attack computation, an inviscid linearstrength vortex airfoil analysis [3], and an empirical direct boundary layer computation [4].

Structural modelling was supplied through an implementation of classical beam theory [5]. This was comprised of a torsional twist prediction and a bending deflection computation. For solution purposes, the wing box was numerically approximated as a swarm of area concentrations that allowed for the rapid determination of the wing box centroid, moments of inertia, and shear centre.

The global solution was produced through a coupling of these solvers. For a given analysis, the implementation would iteratively converge the inviscid loading and structural response loads. Once this was completed, the viscous analysis was run to develop the parasitic drag load and allow for the computation of the wing aerodynamic efficiency. As developed, the analyzer was capable of predicting the dominant aerodynamic loads for the modeled non-rigid wing within acceptable time frames. 


\section{References}

[1] J. Martins and A. Lambe, "Multidisciplinary Design Optimization: A Survey of Architectures," AIAA Journal, vol. 51, no. 9, pp. 1-48, 2013.

[2] A. Elham and M. van Tooren, "Tool for preliminary structural sizing, weight estimation, and aeroelastic optimization of lifting surfaces," Proceedings of the Institution of Mechanical Engineers, vol. 230, no. 2, pp. 280-295, 2015.

[3] J. Katz and A. Plotkin, Low-Speed Aerodynamics, New York: Cambridge University Press, 2001.

[4] G. Fujiwara, D. Chaparro and N. Nguyen, "An Integral Boundary Layer Direct Method Applied to 2D Transonic Small-Disturbance Equations," in AIAA Applied Aerodynamics Conference, Washington DC, 2016.

[5] R. C. Hibbeler, Mechanics of Materials, Pearson Prentice Hall, 2011.

[6] M. Selig, "UIUC Airfoil Coordinates Database," University of Illinois, [Online]. Available: https://mselig.ae.illinois.edu/ads/coord_database.html\#N. [Accessed 2021].

[7] M. Drela, "XFOIL: An Analysis and Design System for Low Reynolds Number Airfoils," Low Reynolds Number Aerodynamics, pp. 1-12, 1989.

[8] N. Curle, The Laminar Boundary Layer Equations, Oxford: Oxfor University Press, 1962.

[9] B. J. Cantwell, "Viscous Flow Along a Wall," Stanford University Department of Aeronautics and Astronautics, [Online].

Available: https://web.stanford.edu/ cantwell/AA200_Course_Material/Ch09_Viscous_Flow_Along_a_Wall.pdf. [Accessed March 2021].

[10] T. H. G. Megson, Aircraft Structures for Engineering Students, Kidlington: Elsevier Ltd., 2017. 


\section{Appendix A: Effective Angle of Attack Derivation}

Given that the induced drag coefficient $C_{d i}$, the global angle of attack $\alpha_{g}$, and twist angle $\phi$ were known, and that $\alpha_{i}$ was unknown.

The effective angle of attack was defined with the following equation:

$$
\alpha_{e f f}=\alpha_{g}+\phi-\alpha_{i}
$$

Using the linear-lift assumption from Thin Airfoil Theory, the lift coefficient function was defined as:

$$
C_{l}(\alpha)=2 \pi \alpha+C_{l 0}
$$

Where $\alpha$ was some angle of attack in radians, and $C_{l 0}$ was the zero angle-of-attack lift coefficient of the aircraft. The $C_{l 0}$ was assumed to be a known value.

The induced drag coefficient was defined with the following equation:

$$
C_{d i}=C_{l}\left(\alpha_{e f f}\right) \cdot \sin \alpha_{i}
$$

Where $C_{d i}$ was the induced drag coefficient.

Equation (A.3) was rearranged into the following form, and equation (A.2) was substituted in:

$$
0=\left(2 \pi \alpha_{e f f}+C_{l 0}\right) \sin \alpha_{i}-C_{d i}
$$

Equation (A.1) was rearranged and substituted in for $\alpha_{i}$. This produced the final equation used for determination of the effective angle of attack:

$$
0=\left(2 \pi \alpha_{e f f}+C_{l 0}\right) \sin \left(\alpha_{g}+\phi-\alpha_{e f f}\right)-C_{d i}
$$




\section{Appendix B: Shear Center Arm Derivation}

Given the arbitrary cross section presented in the following figure of an arbitrary cross section for a thin member:

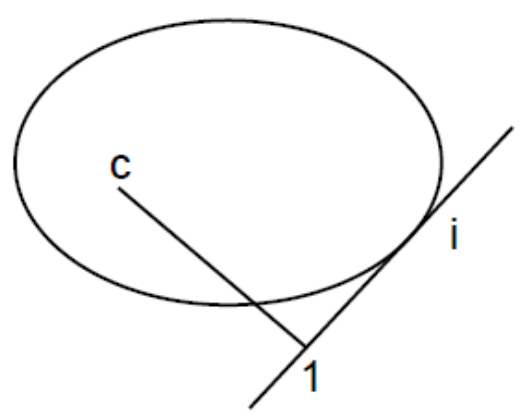

Three points where defined. Point $c$ was located at the centroid, point $i$ was located on the edge of the thin member, and point 1 was set where the lever arm met the tangent line. The distance between point $c$ and point 1 was defined as the lever arm distance with the following equation:

$$
p=\sqrt{\left(x_{1}-x_{c}\right)^{2}+\left(z_{1}-z_{c}\right)^{2}}
$$

The tangent line was defined as having the slope $m=d z / d x$, which permitted the following two definitions for the tangent and lever lines:

$$
\begin{aligned}
& \frac{z_{1}-z_{i}}{x_{1}-x_{i}}=m \\
& \frac{z_{1}-z_{c}}{x_{1}-x_{c}}=\frac{1}{m}
\end{aligned}
$$

Equation (B.3) was rearranged and substituted into equation (B.1) to produce the following relation.

$$
p=\sqrt{m^{2}\left(z_{1}-z_{c}\right)^{2}+\left(z_{1}-z_{c}\right)^{2}}
$$

Equation (B.4) was simplified into the following form:

$$
p=\sqrt{m^{2}+1}\left|z_{1}-z_{c}\right|
$$

Equations (B.2) and (B.3) were rearranged into the following forms:

$$
\begin{aligned}
& z_{1}=m\left(x_{1}-x_{i}\right)+z_{i} \\
& x_{1}=m\left(z_{1}-z_{c}\right)+x_{c}
\end{aligned}
$$

Equation (B.7) was substituted into equation (B.6) to produce the following formula.

$$
z_{1}=\frac{m x_{c}-m^{2} z_{c}-m x_{i}+z_{i}}{1+m^{2}}
$$


Setting $x_{c}=0$ and $z_{c}=0$ as the centroid was used the origin for the reference frame. Equations (B.5) and (B.8) combined to become:

$$
p=\left|\frac{z_{i}-m x_{i}}{1-m^{2}}\right| \sqrt{m^{2}+1}
$$

Substituting the definition of $m$ yielded the final equation below:

$$
p=\left|\frac{z_{i}-\frac{d z}{d x} x_{i}}{1-\left(\frac{d z}{d x}\right)^{2}}\right| \sqrt{\left(\frac{d z}{d x}\right)^{2}+1}
$$




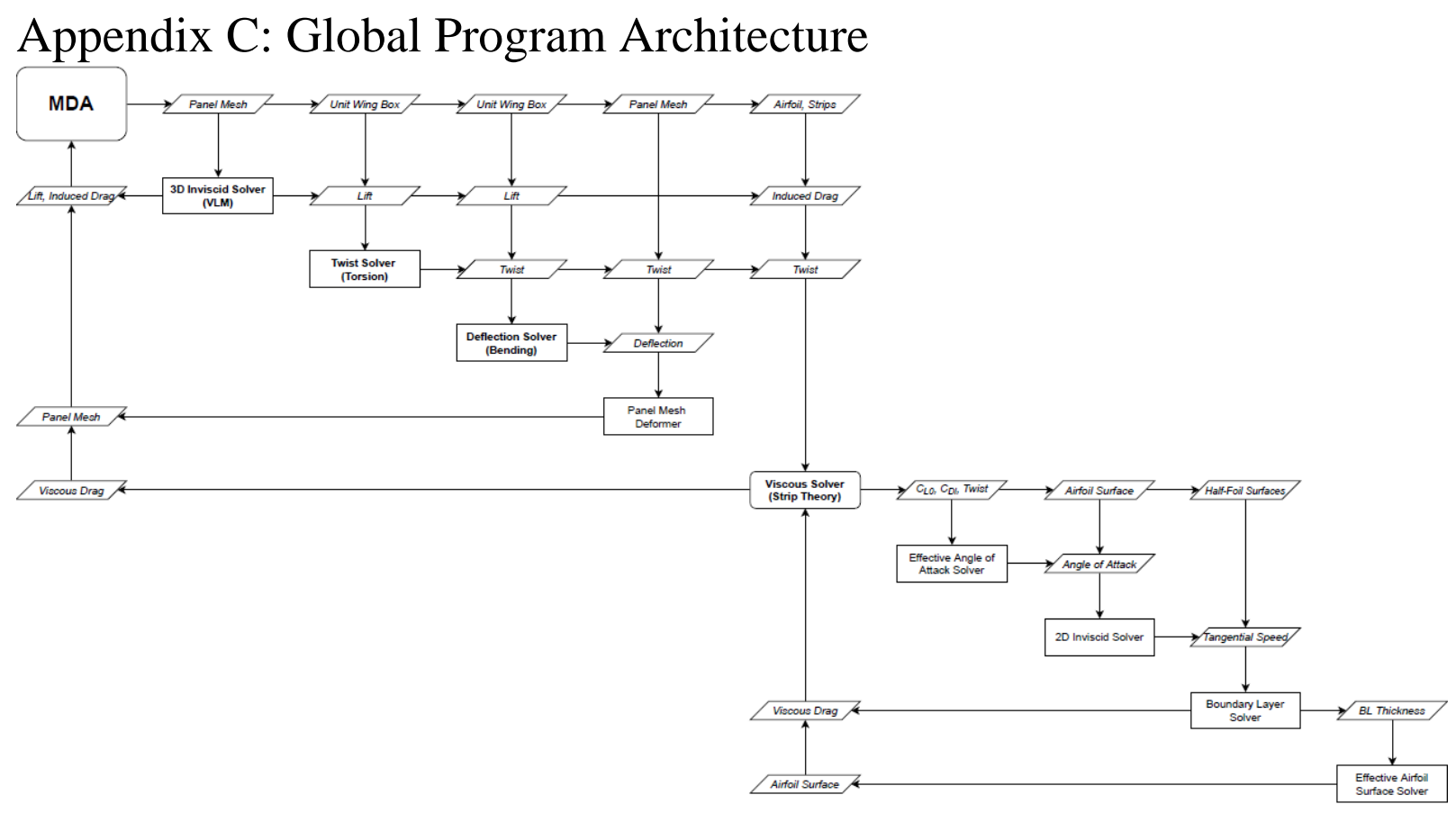




\section{Appendix D: Main MATLAB Function}

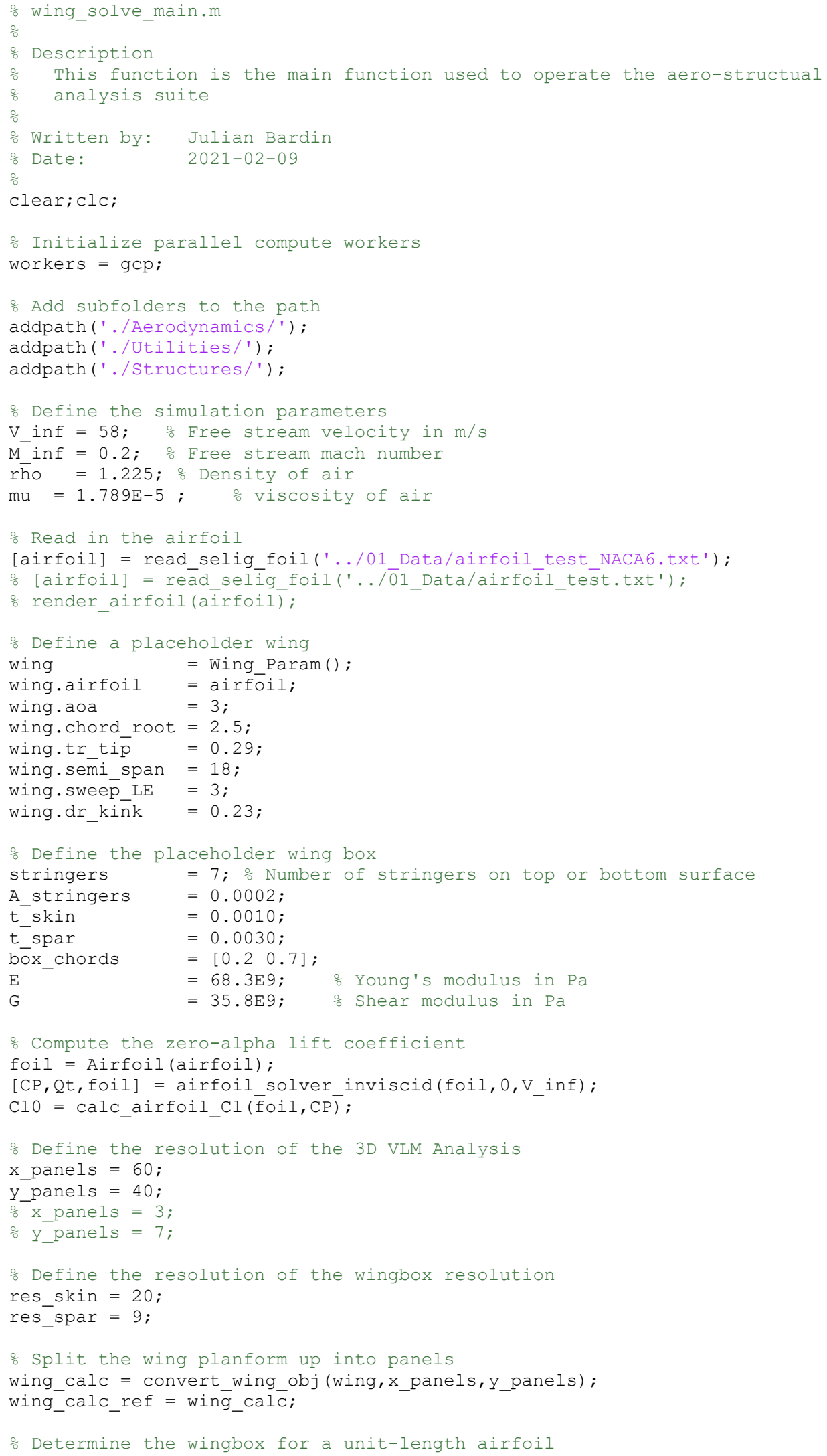




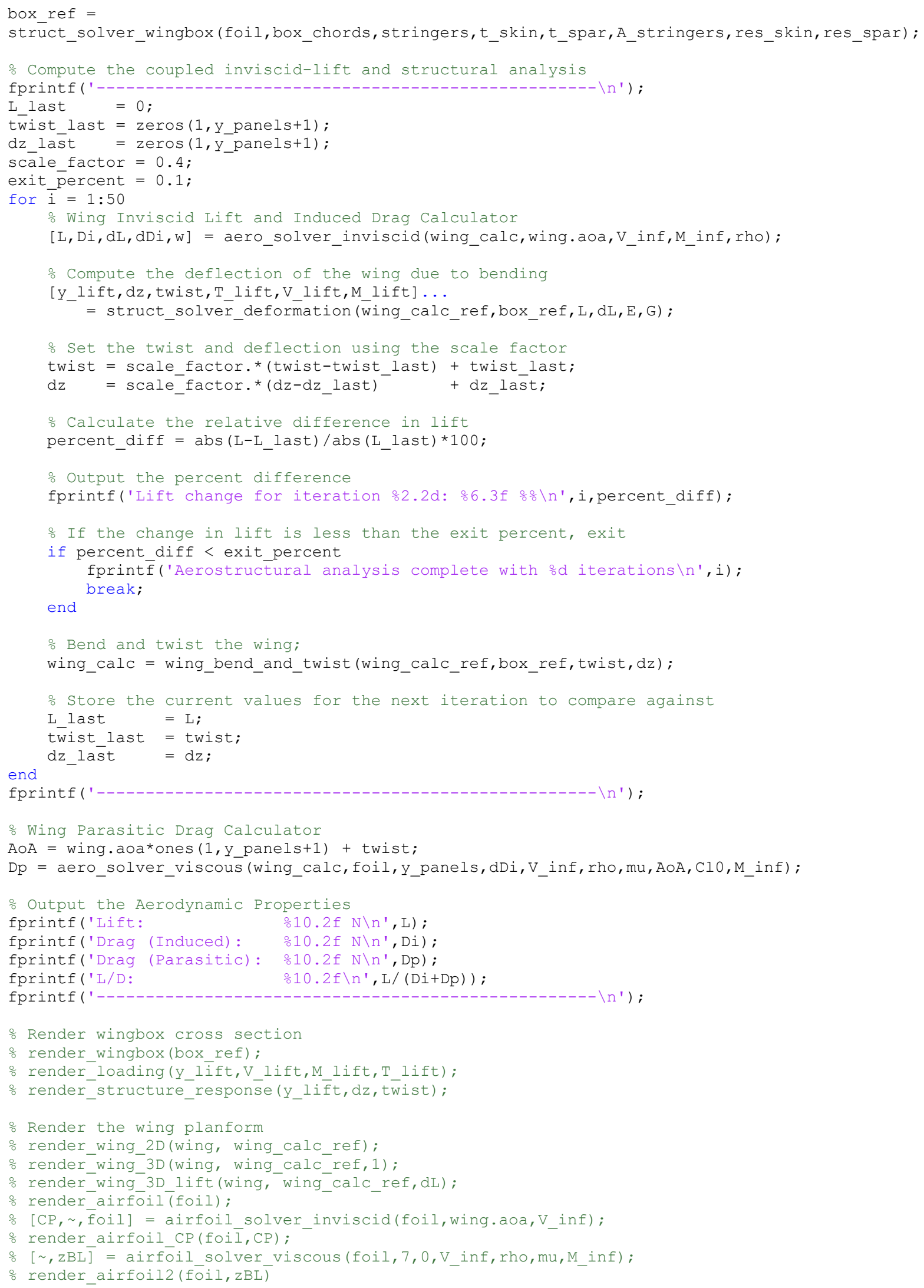




\section{Appendix E: Aerodynamic Functions}

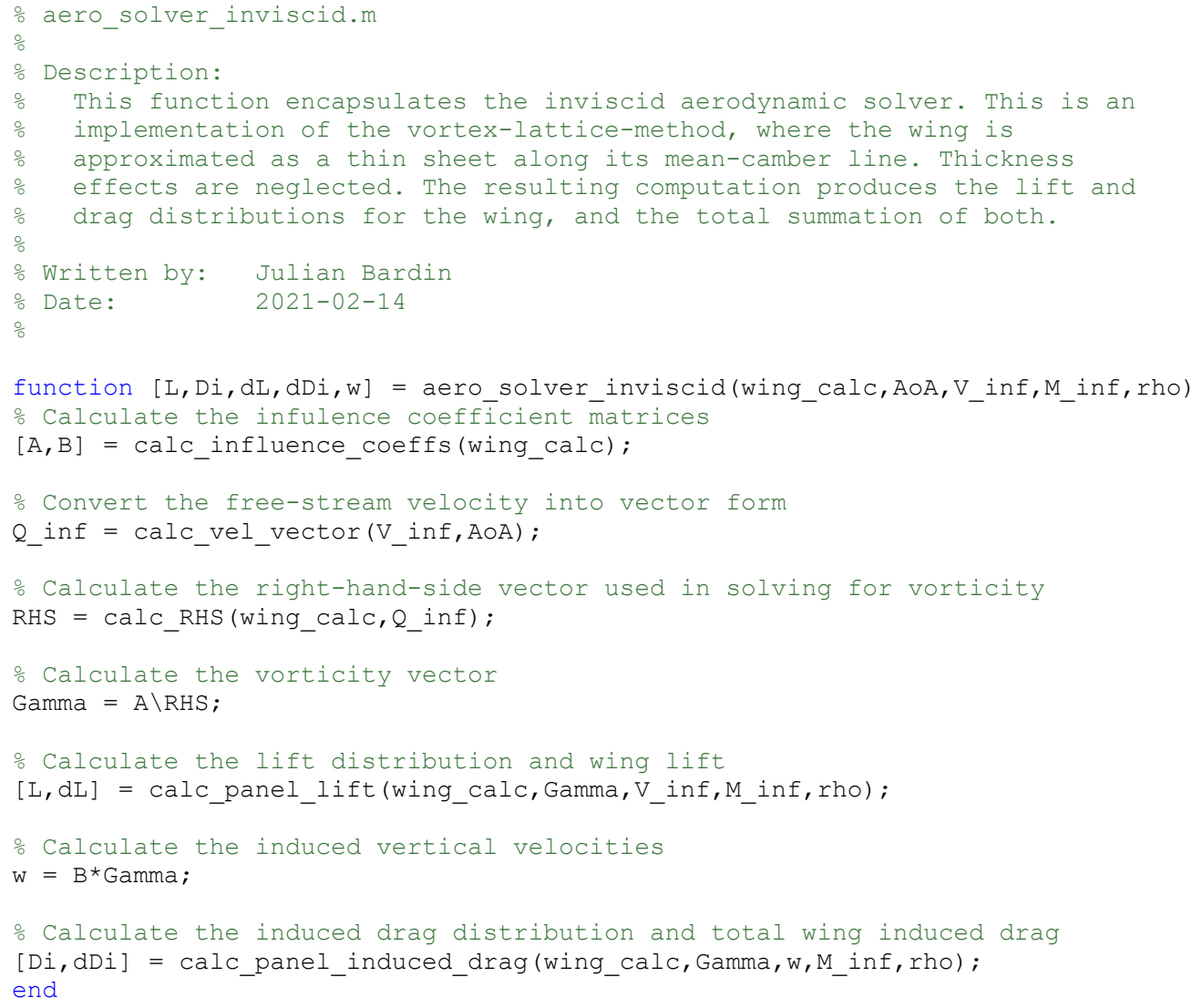




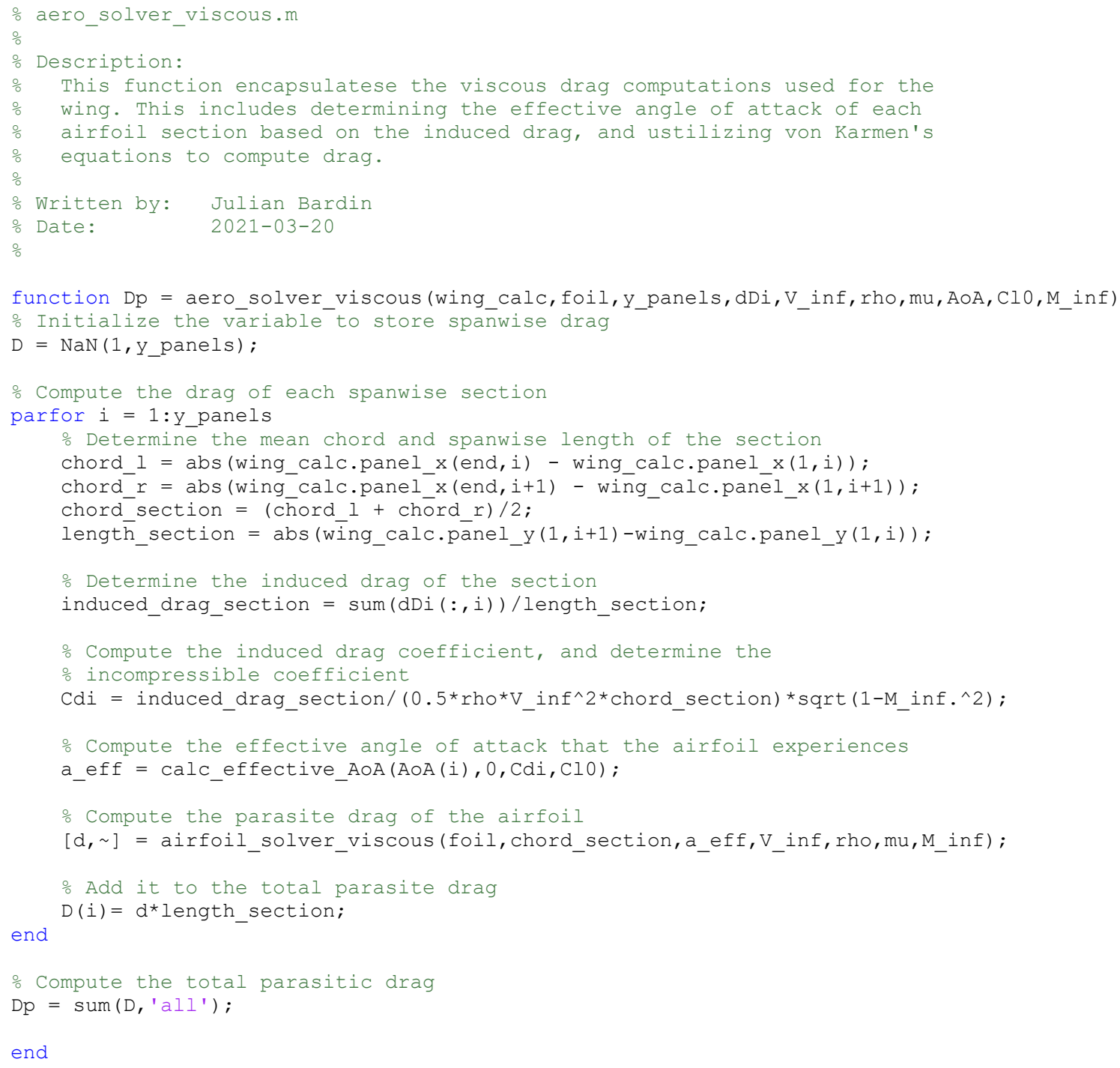




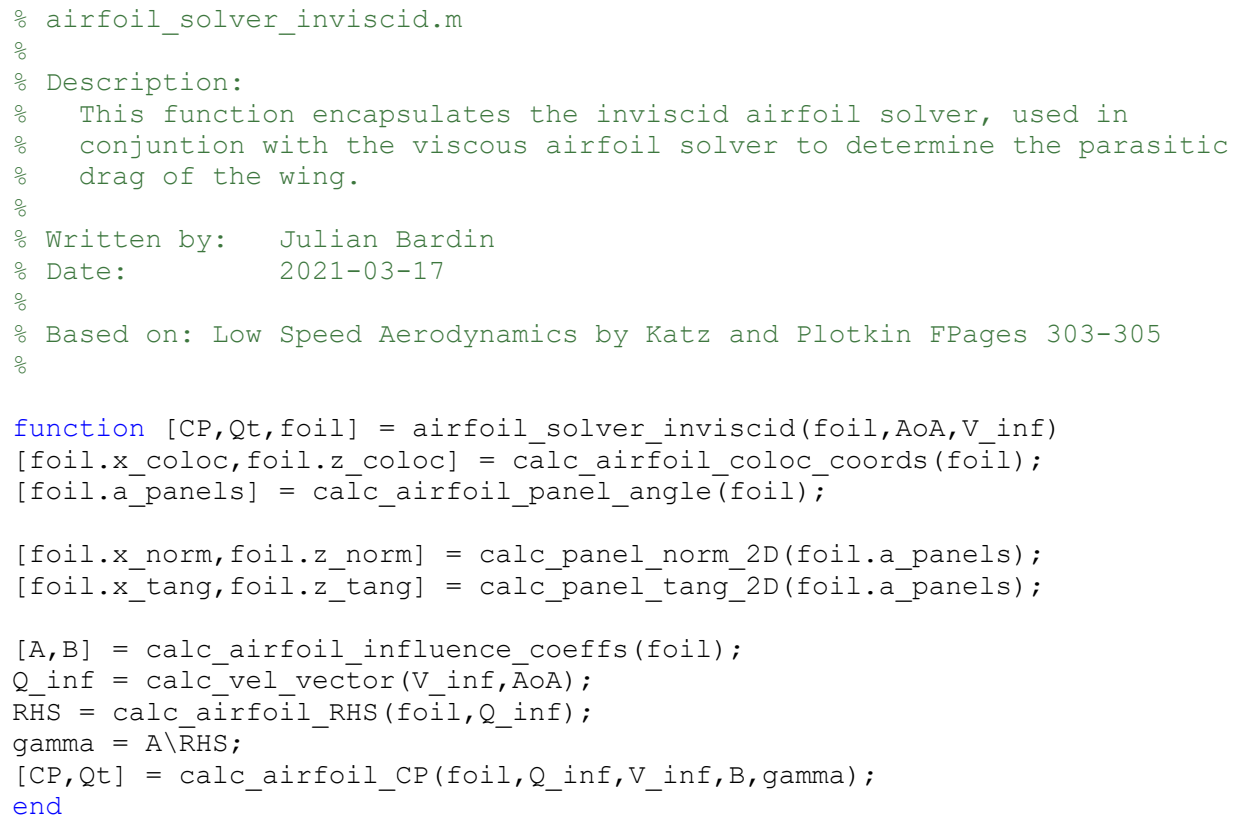




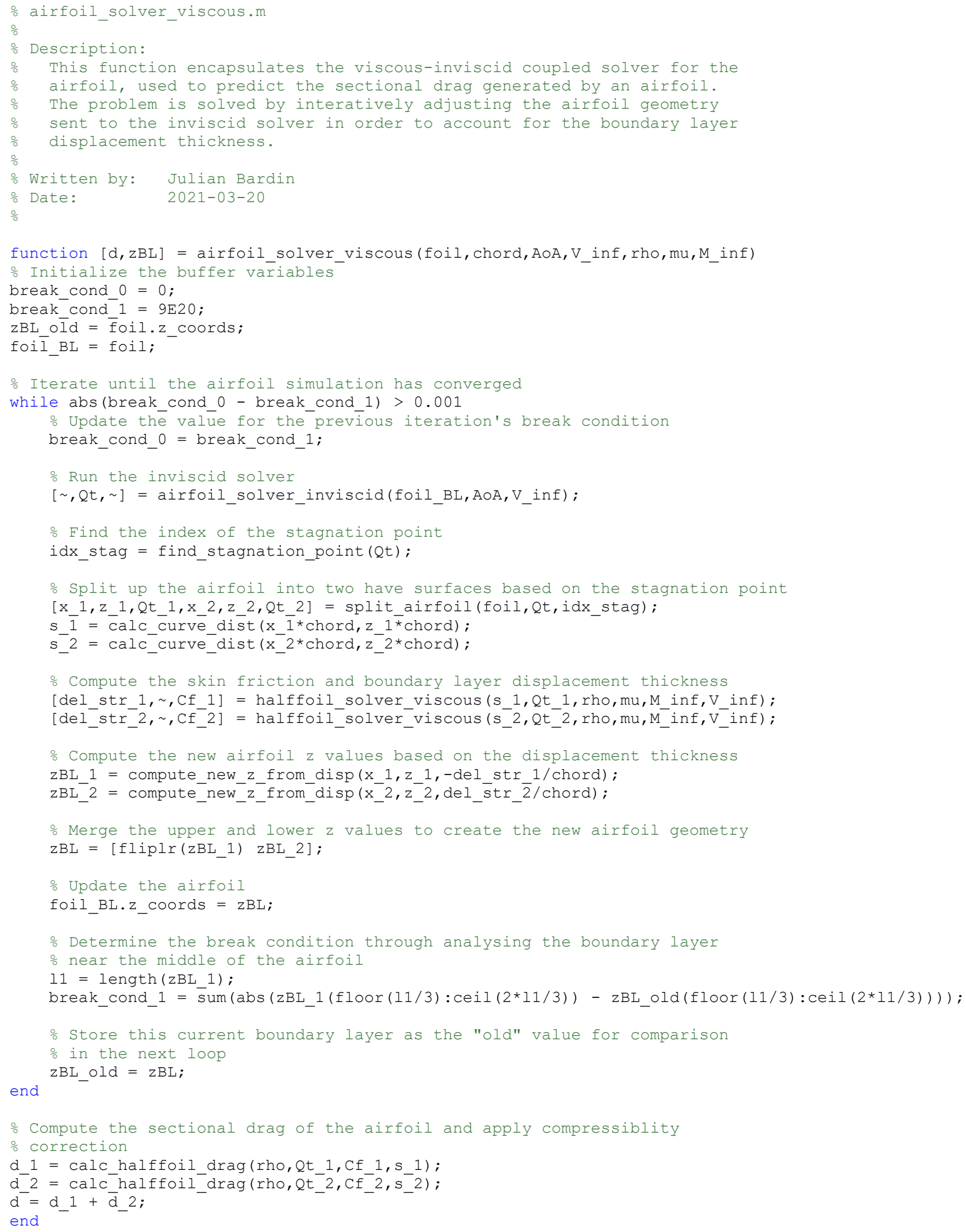




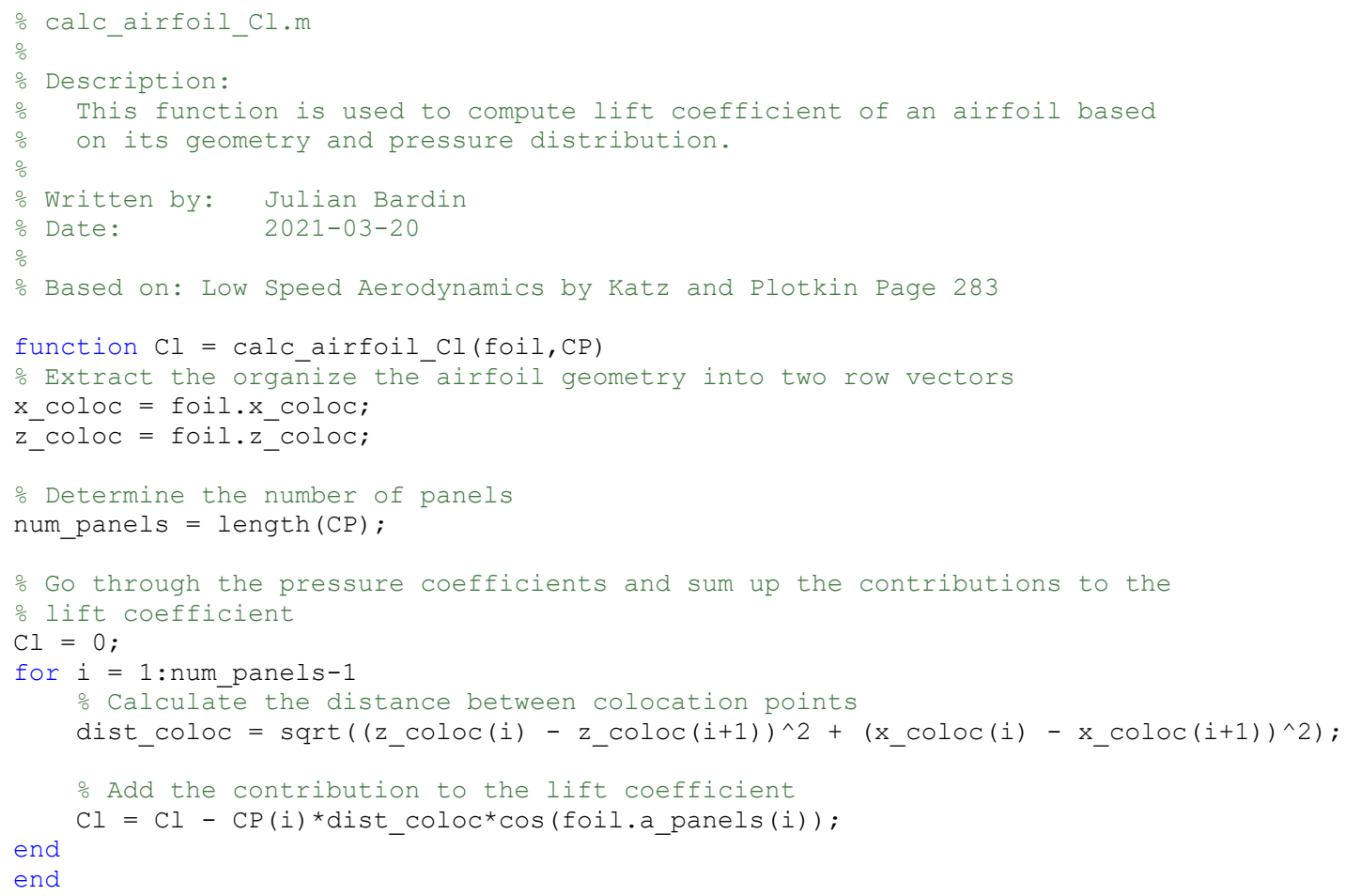




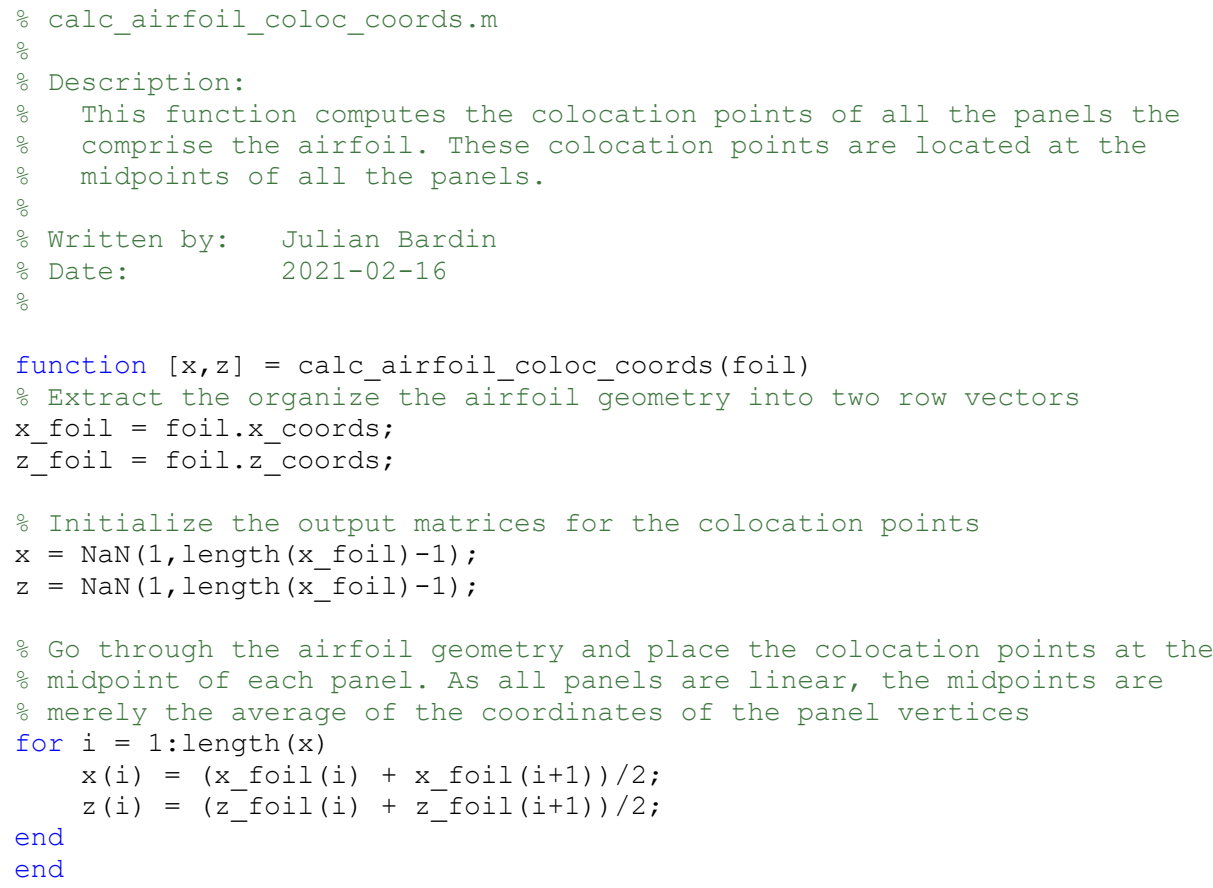




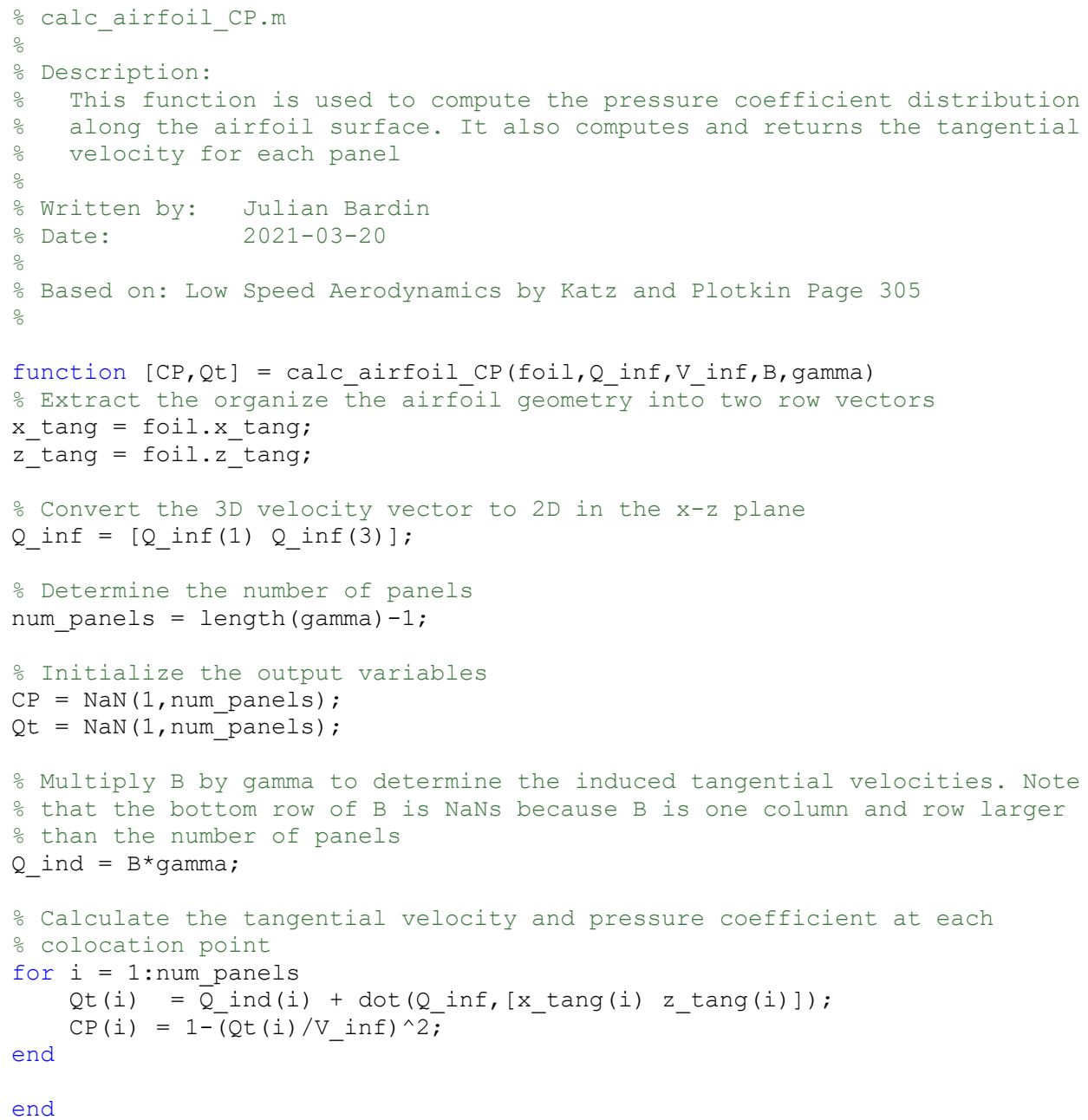




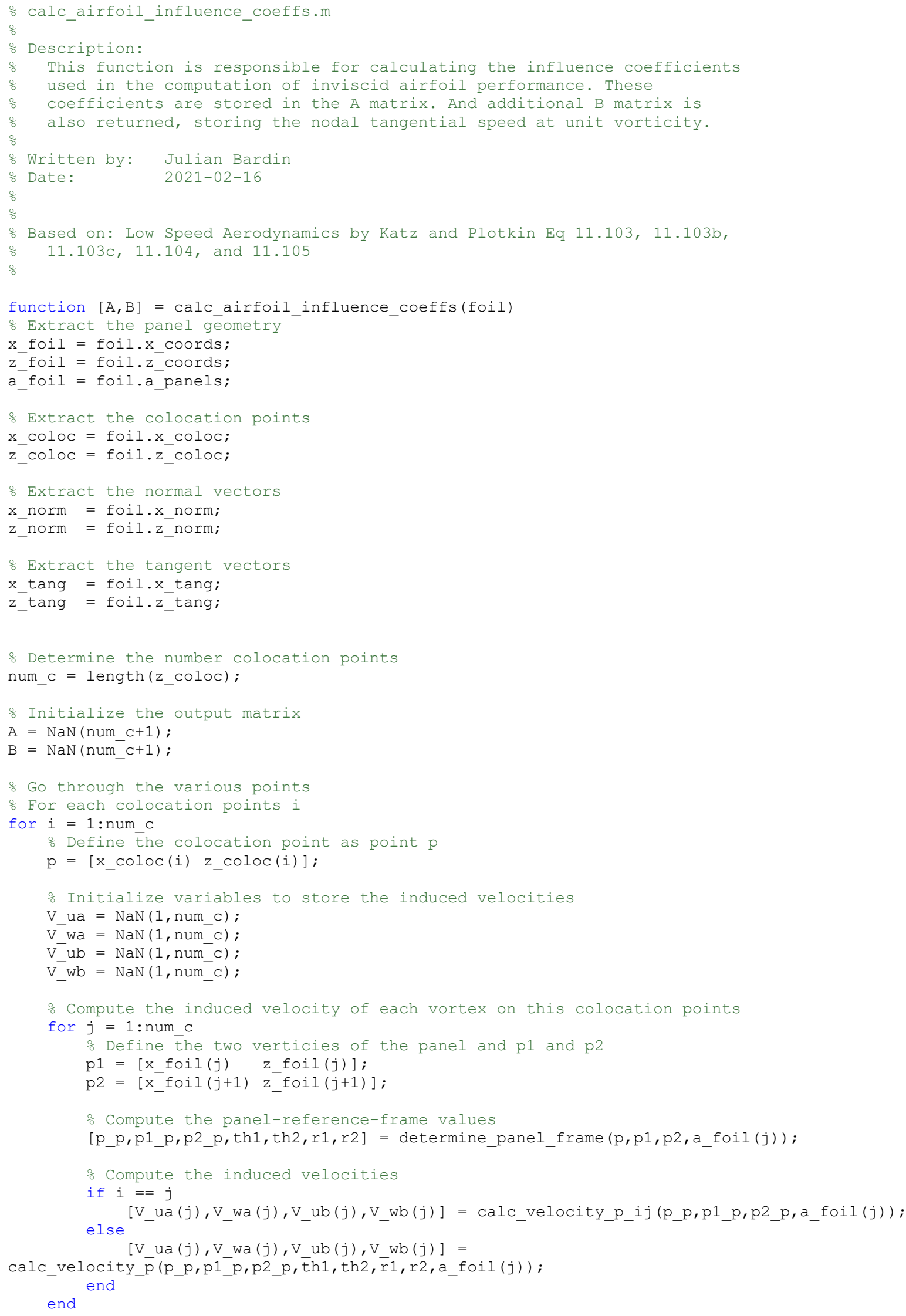




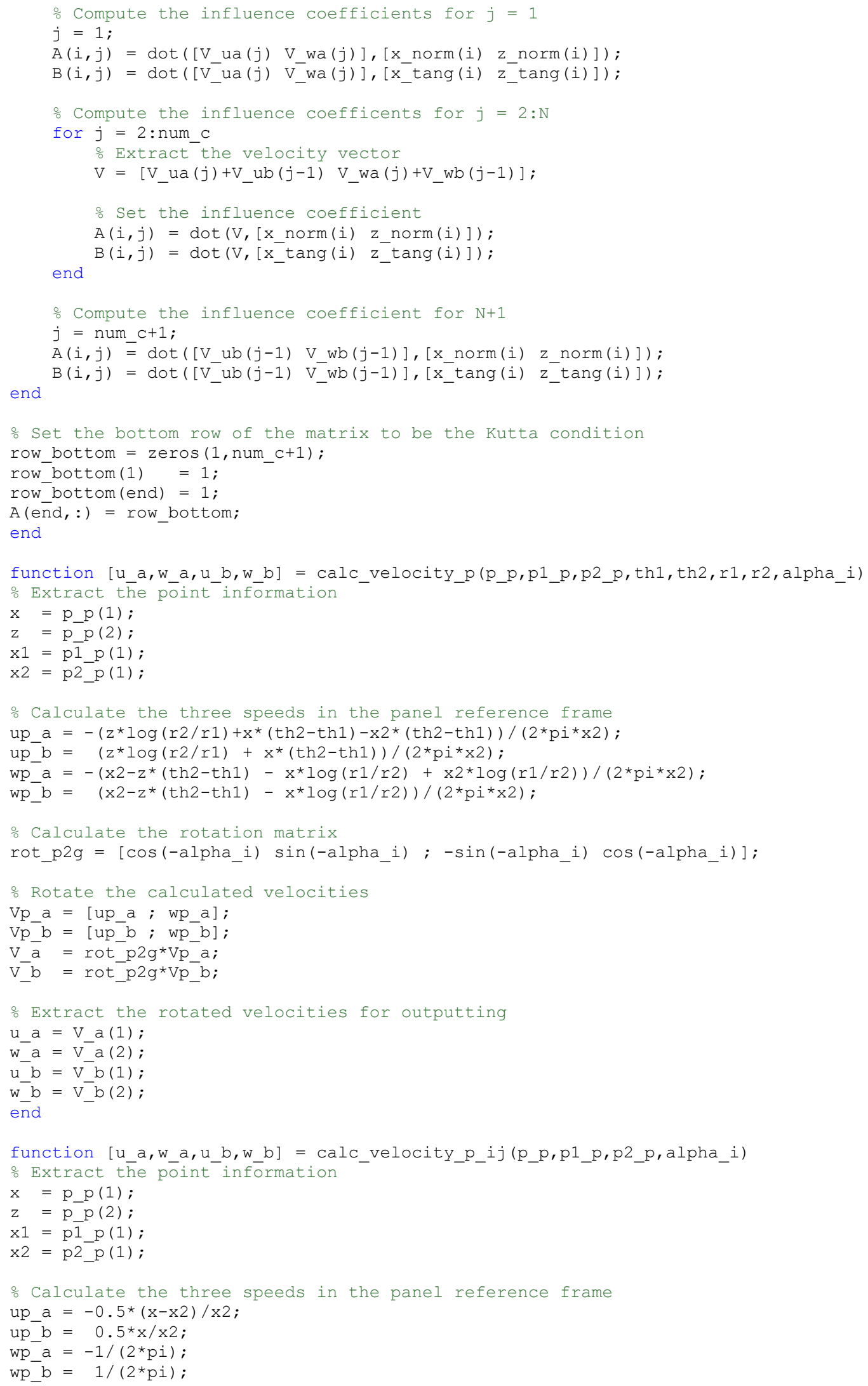




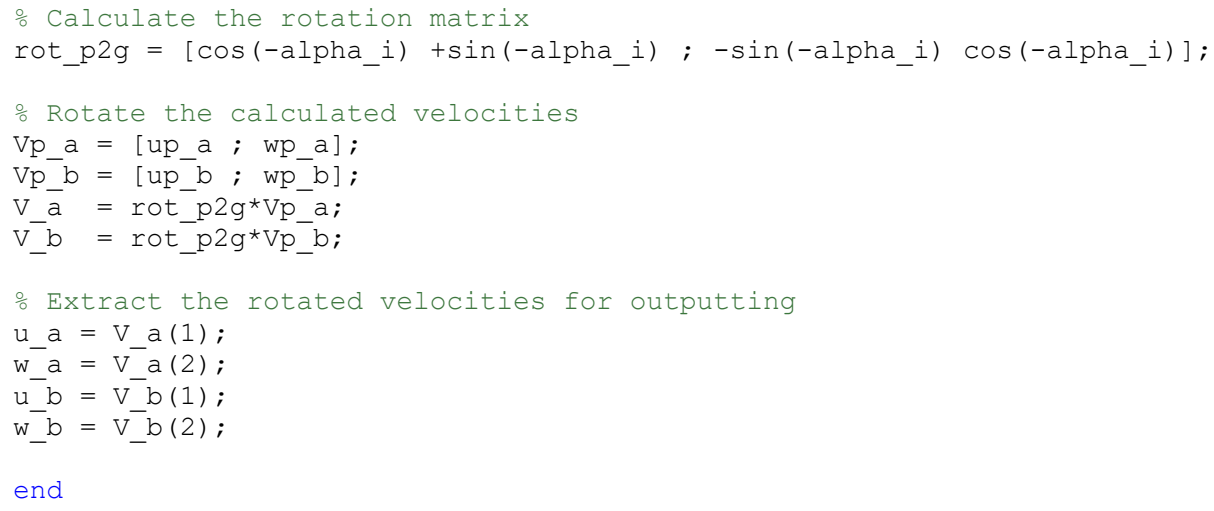




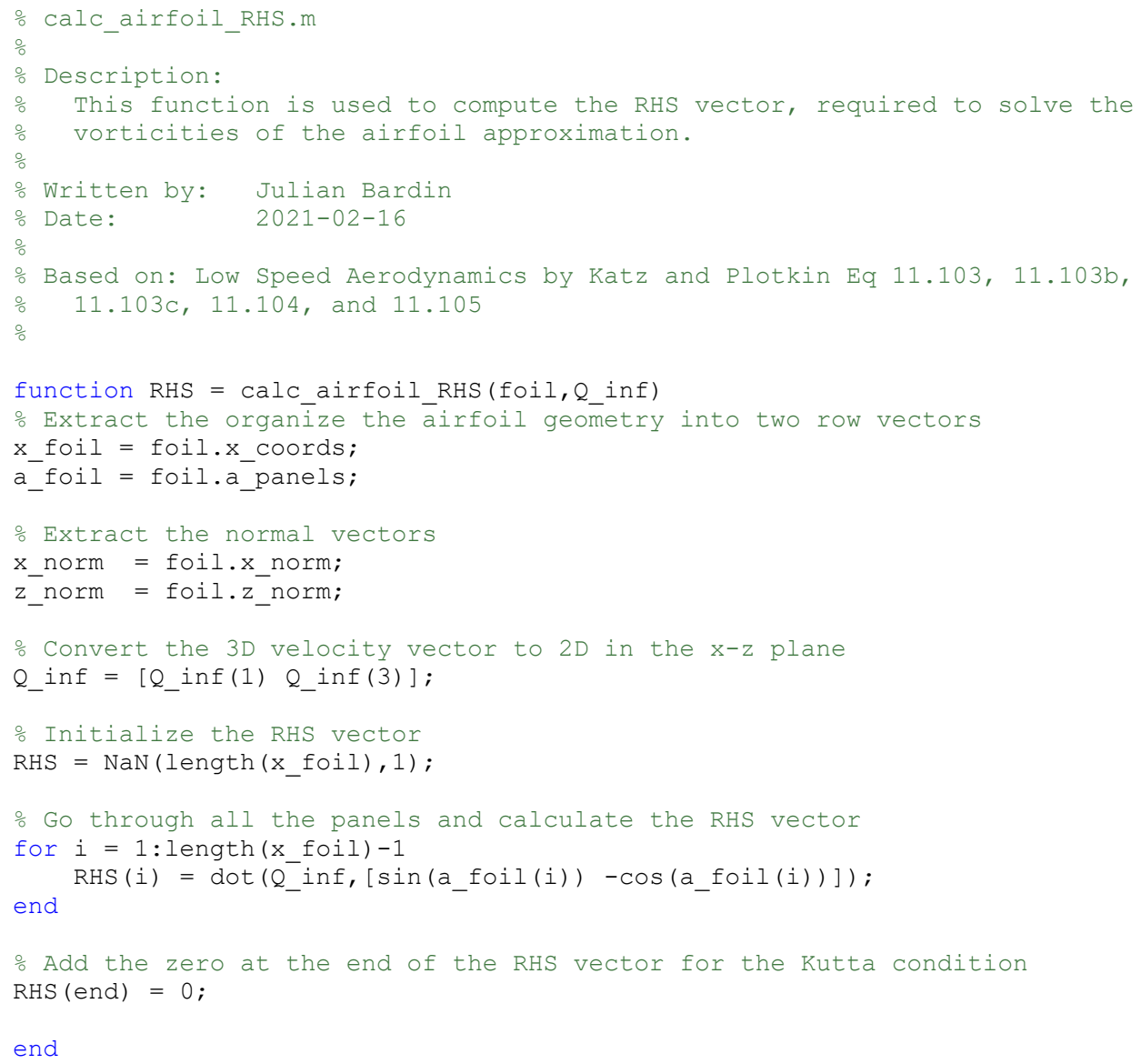




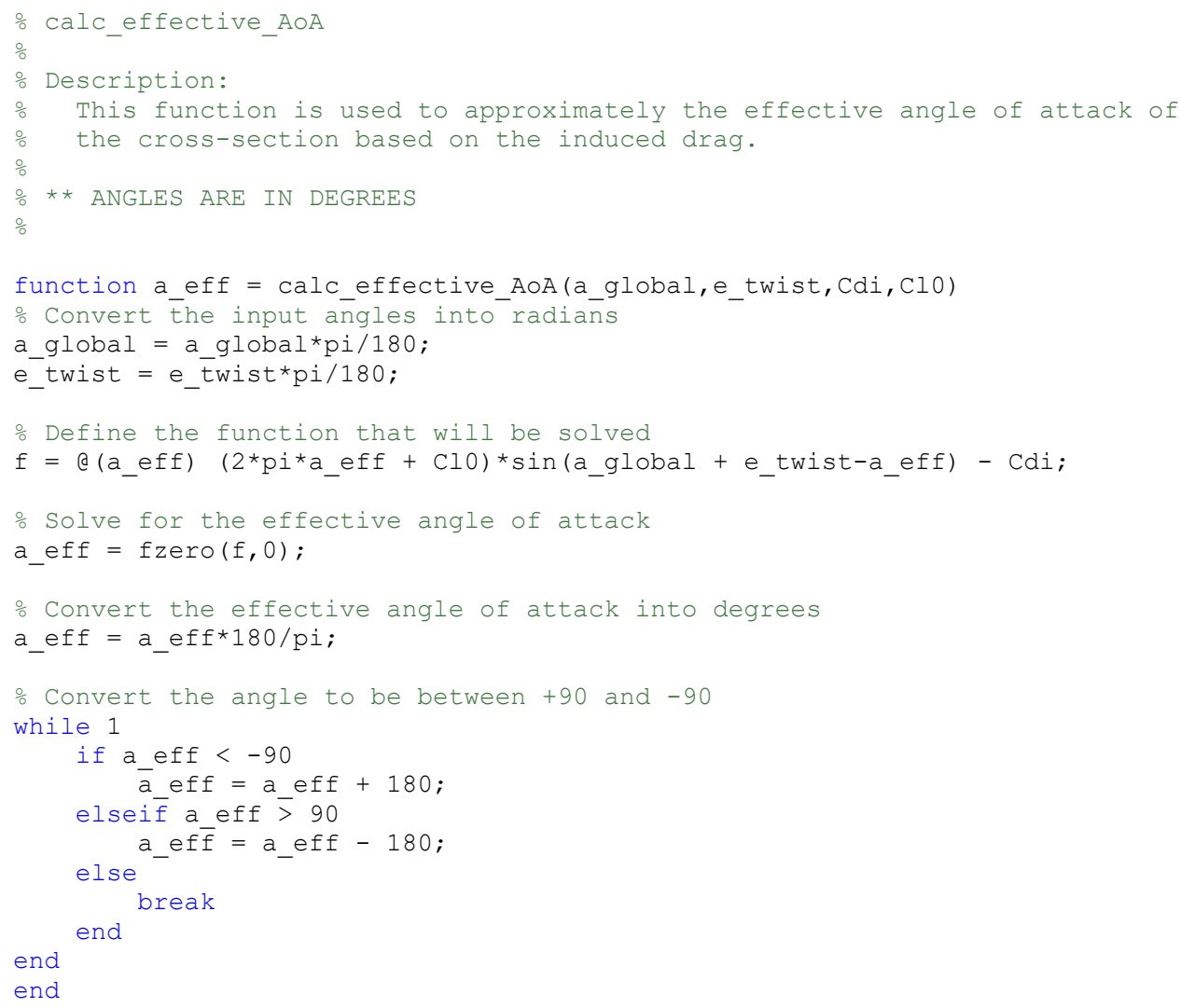




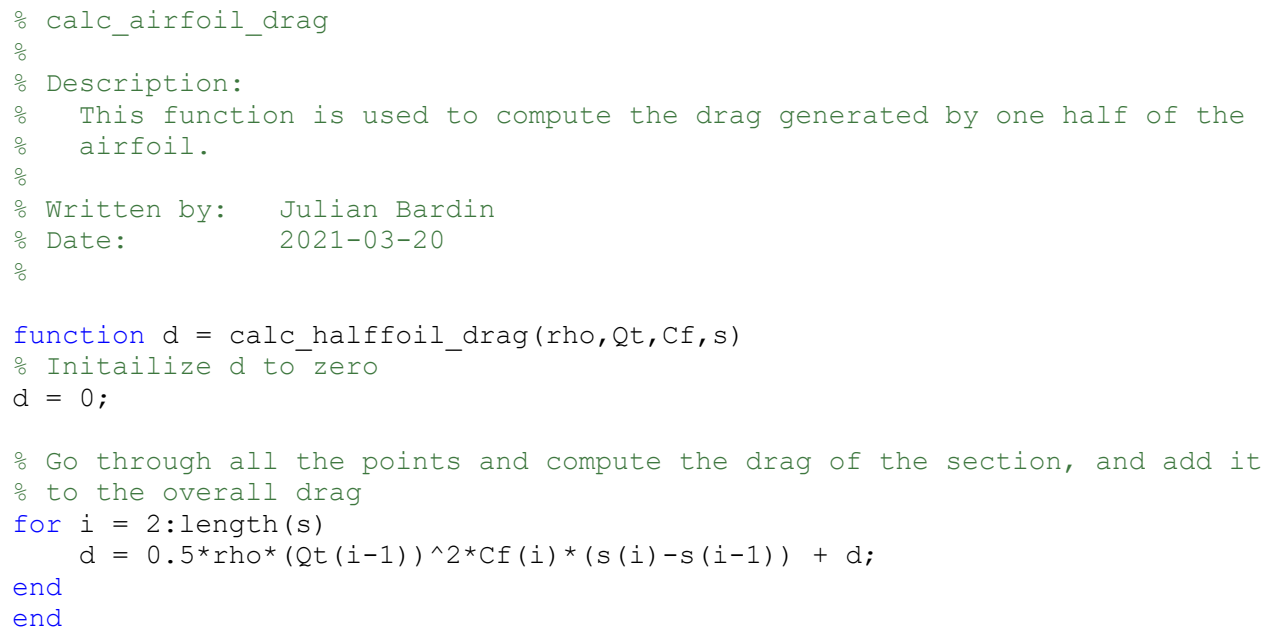




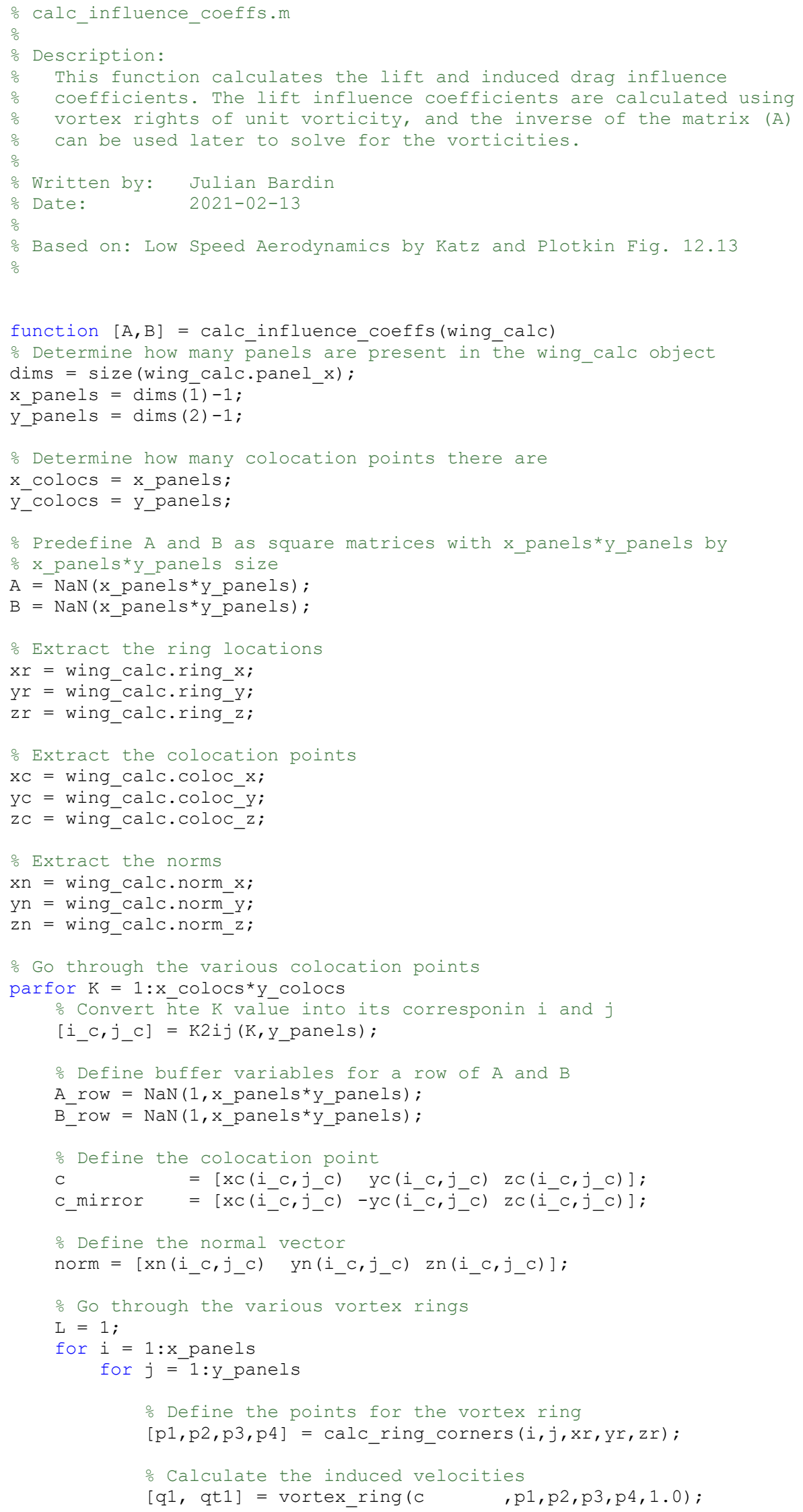




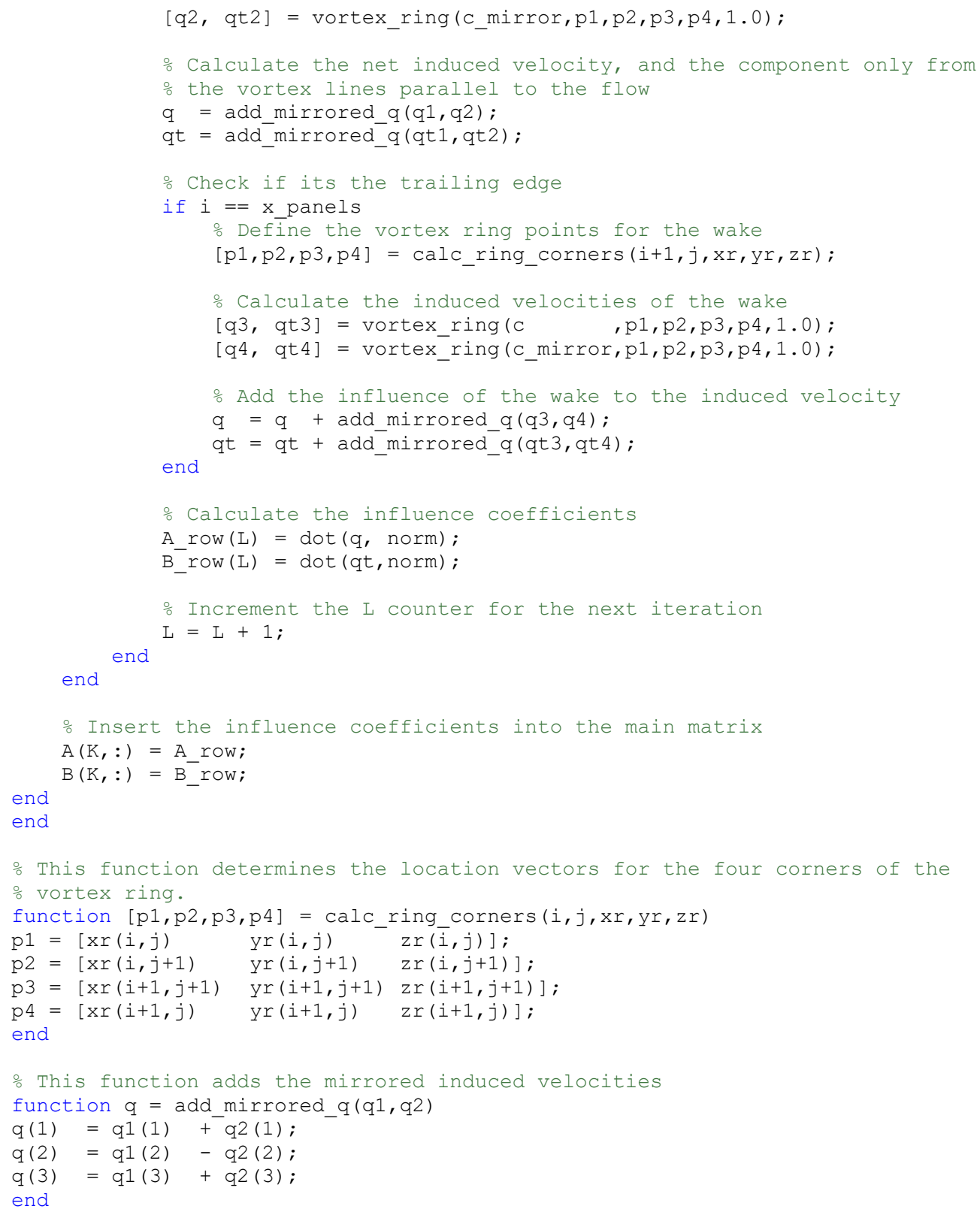




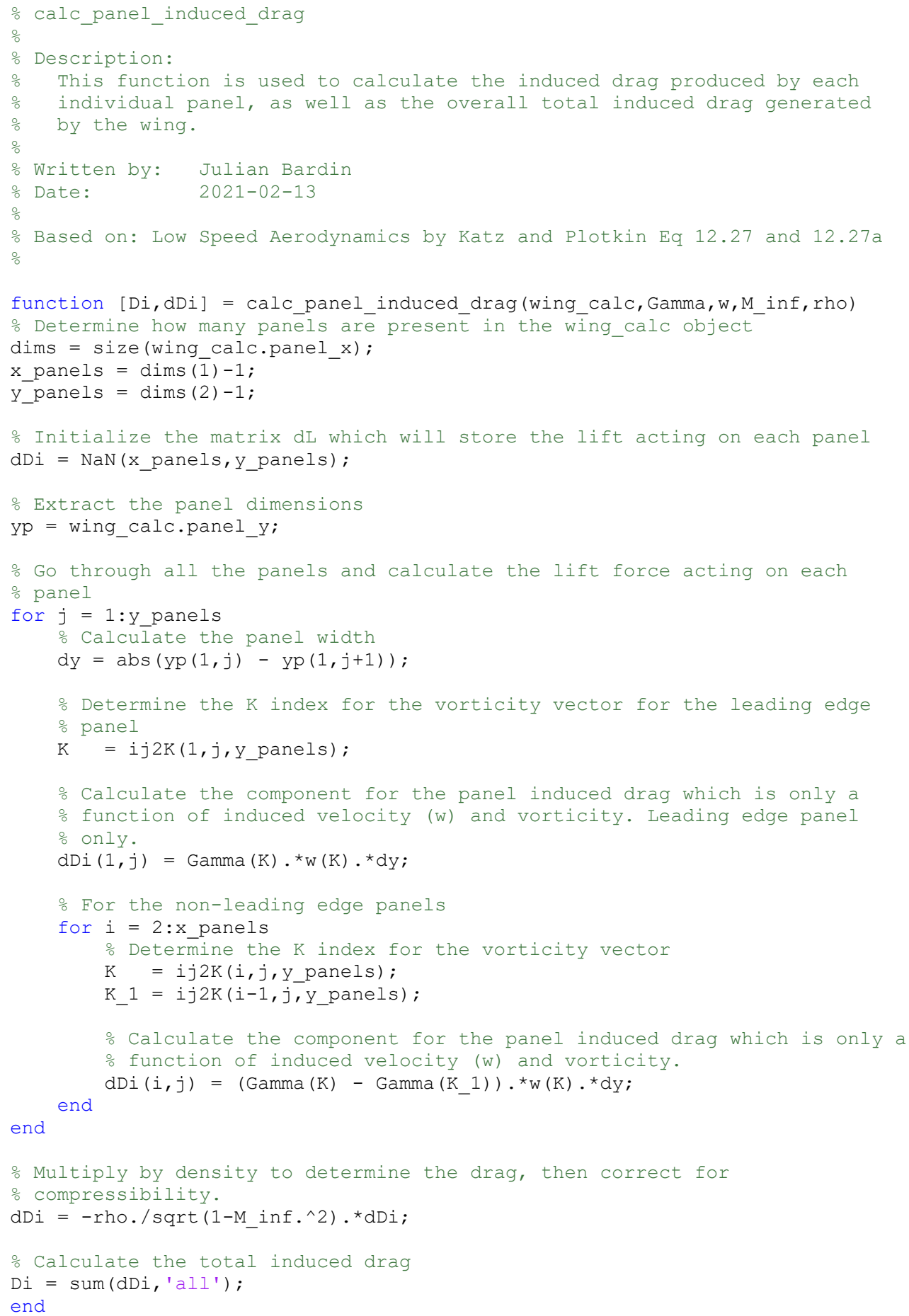




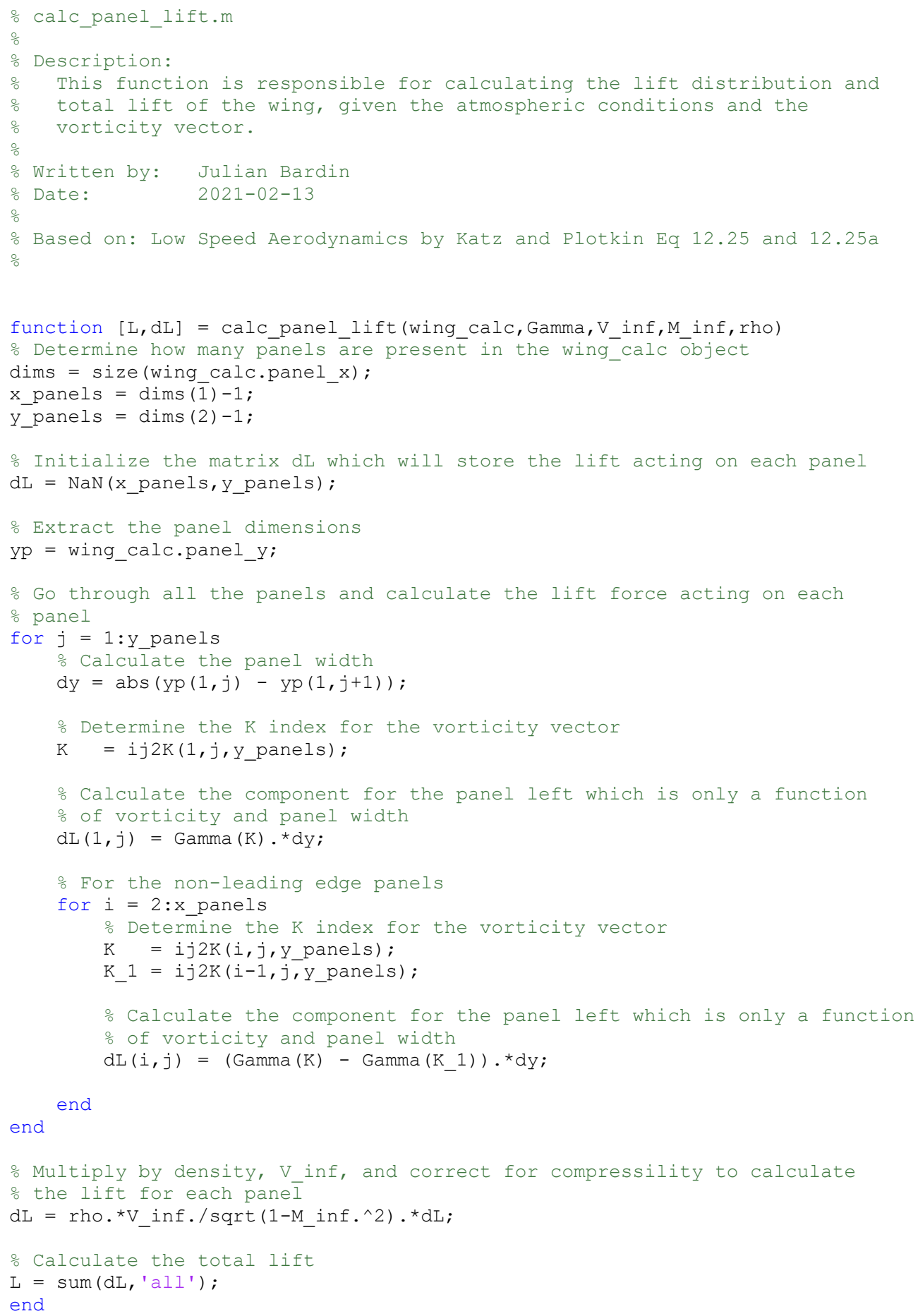




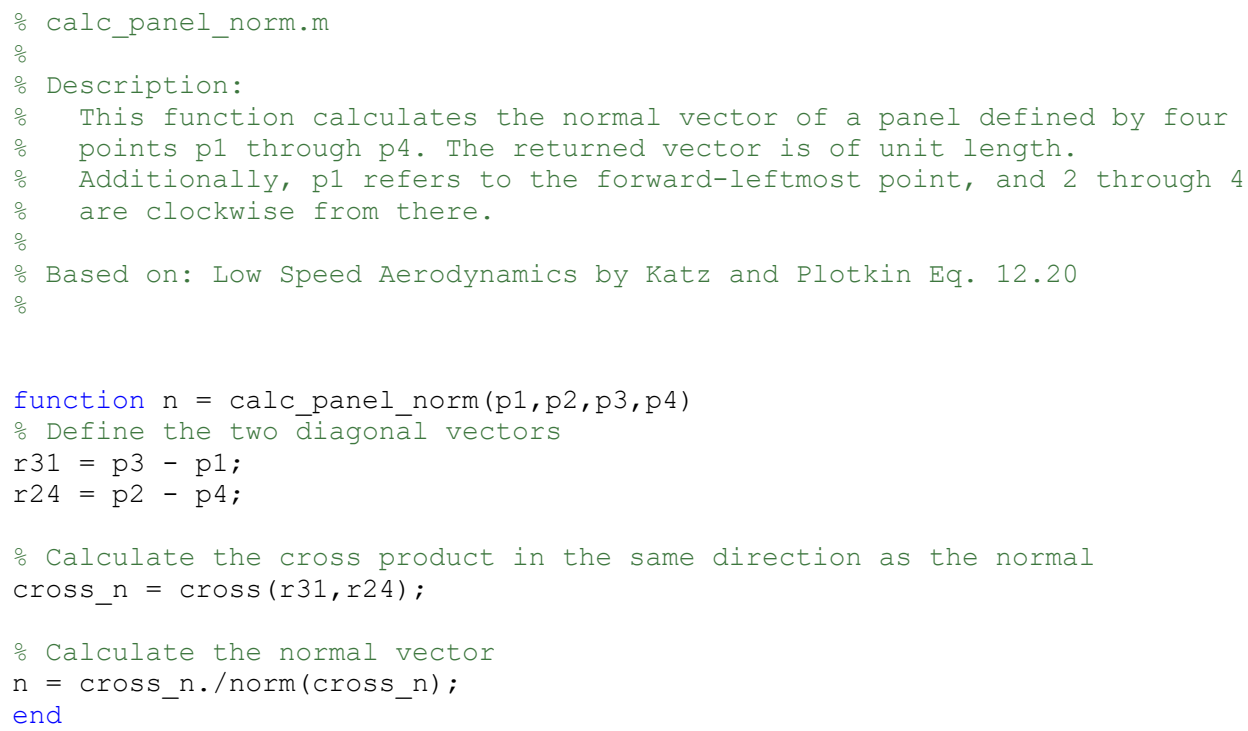




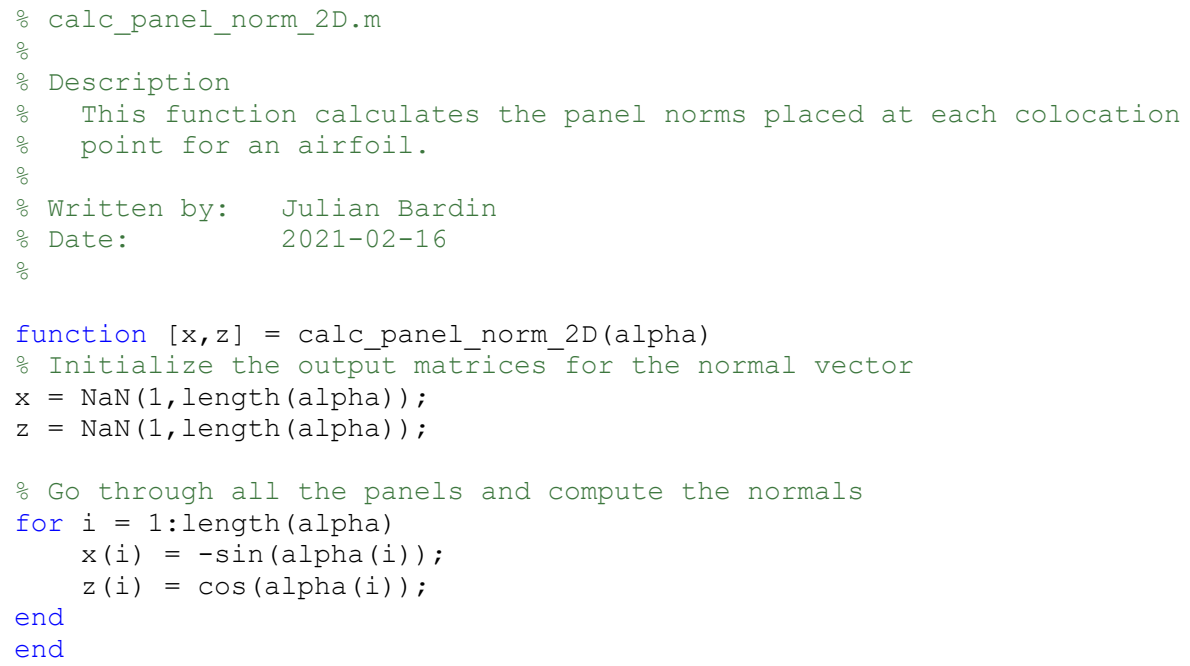




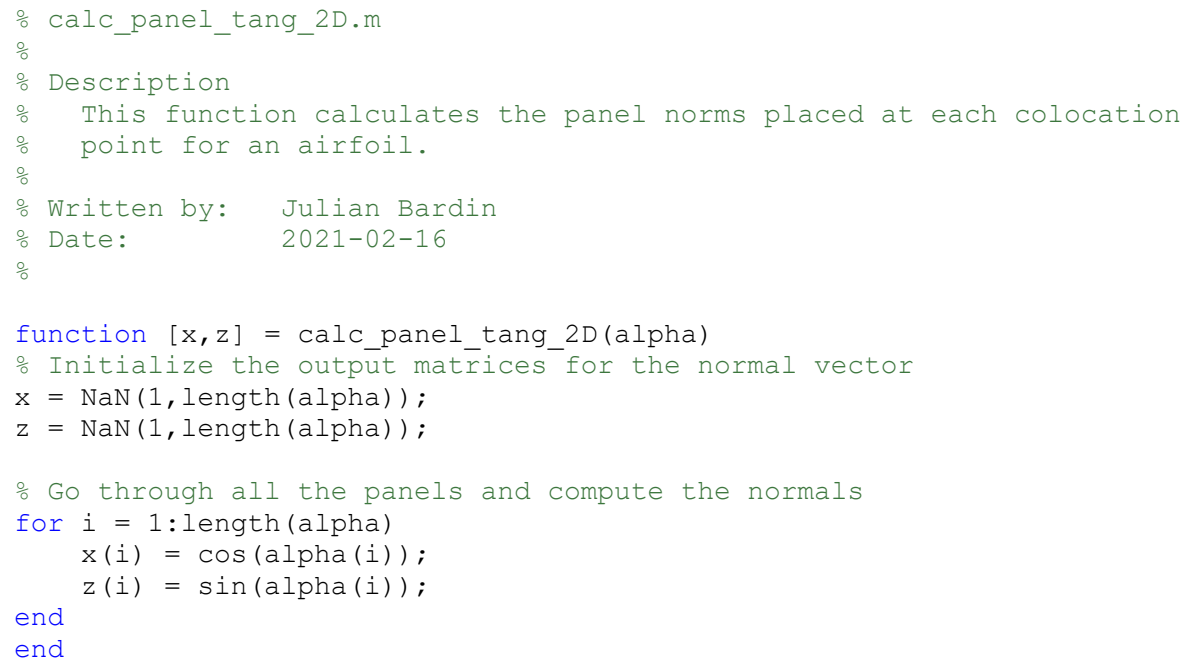




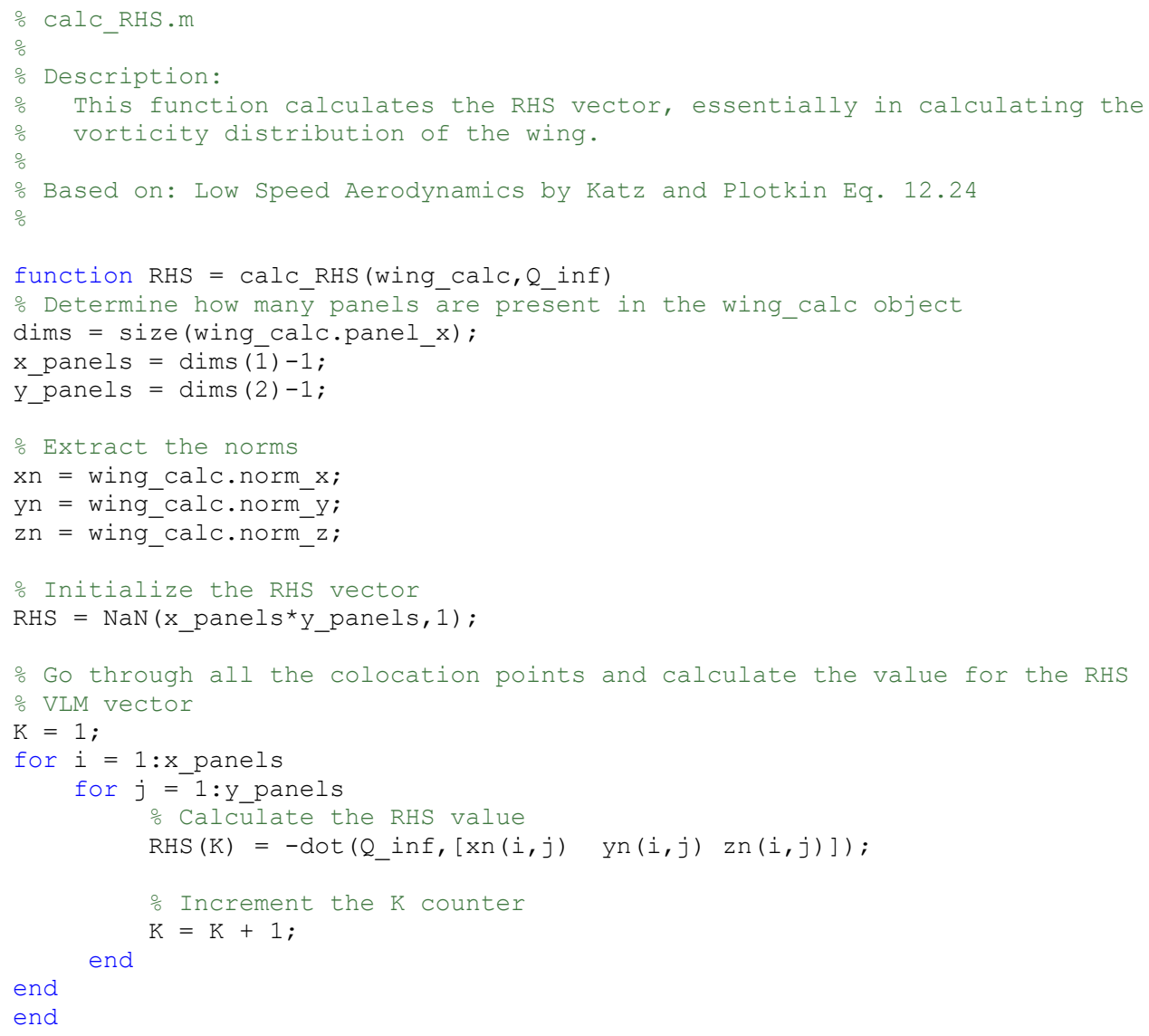




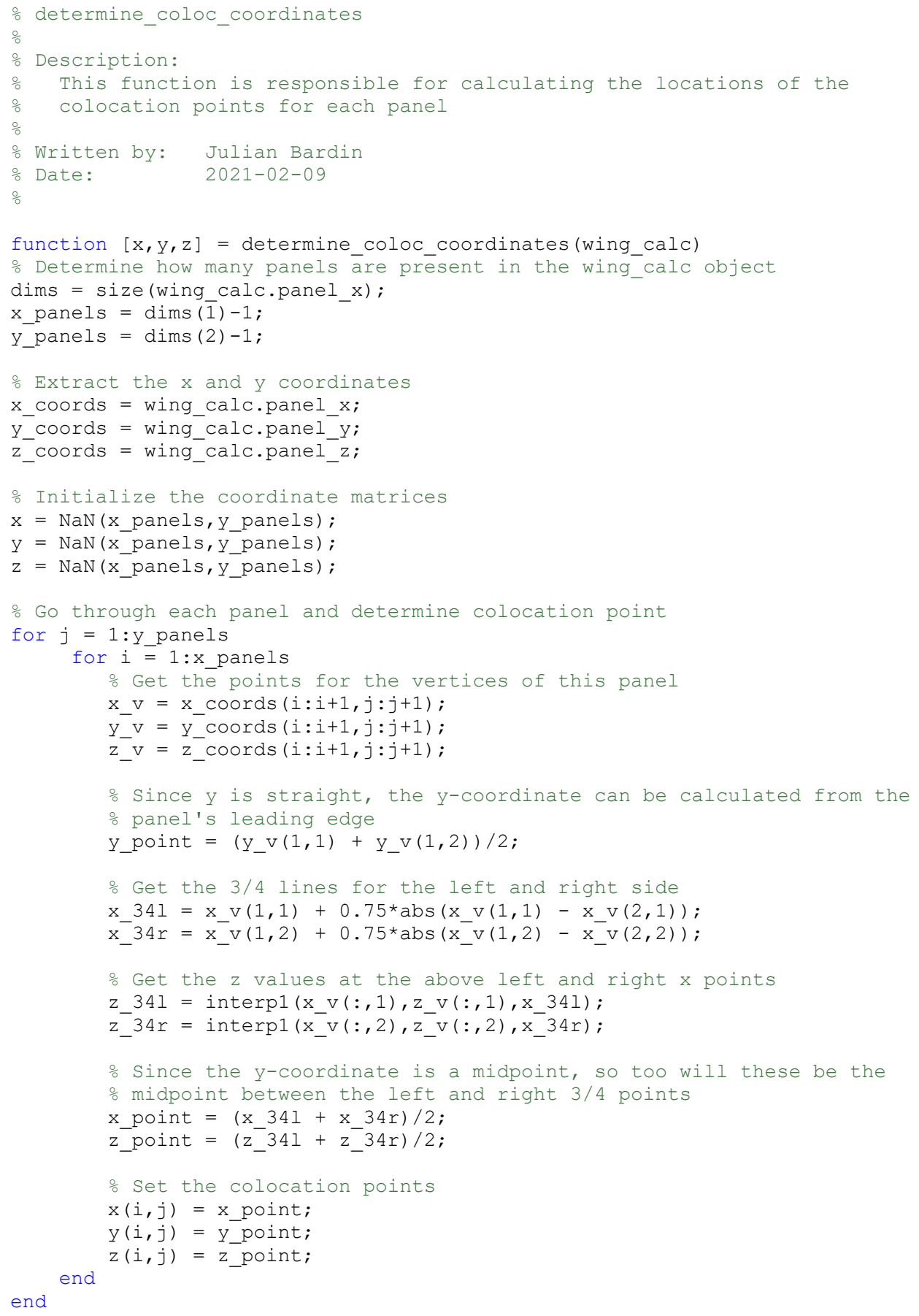




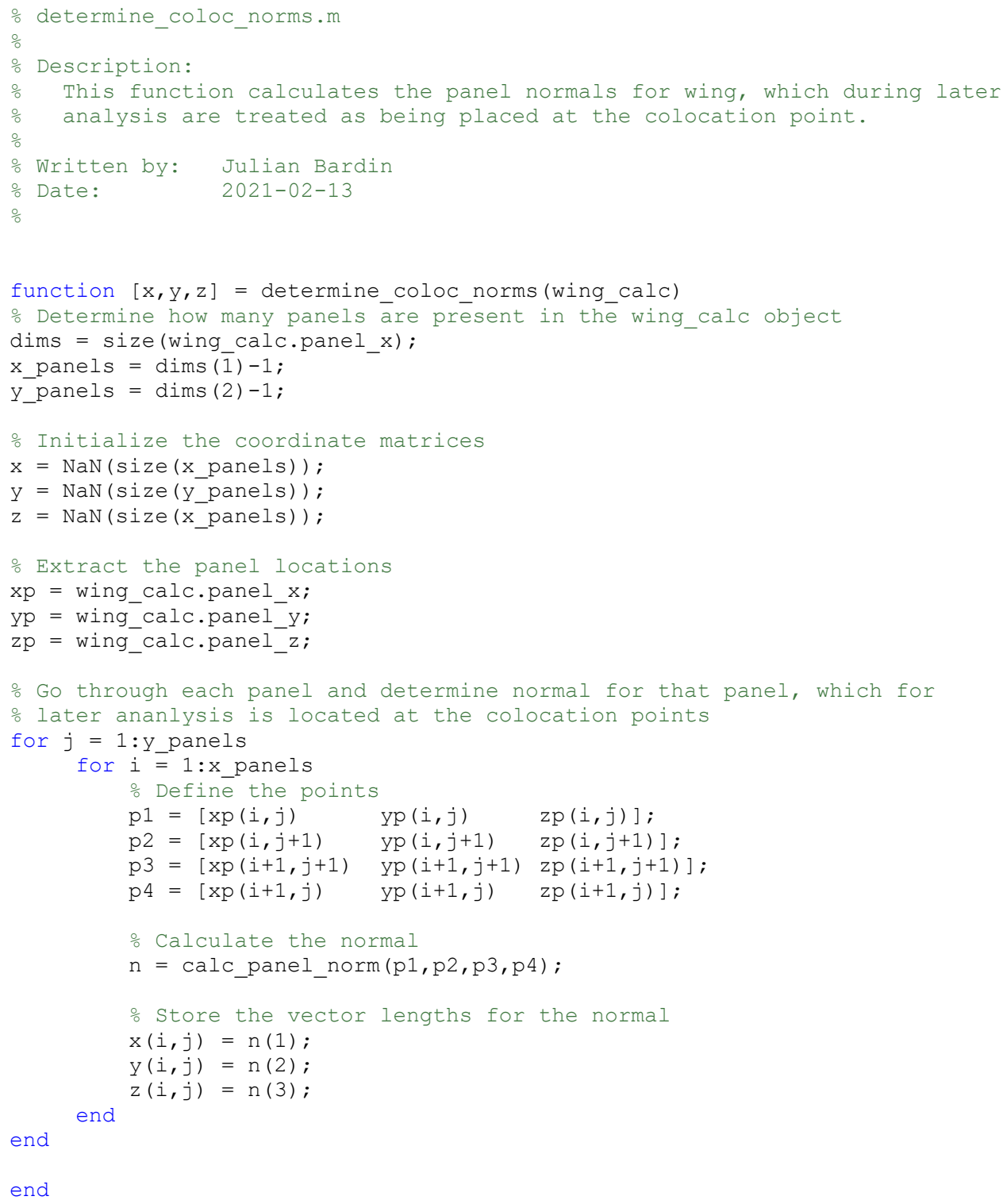




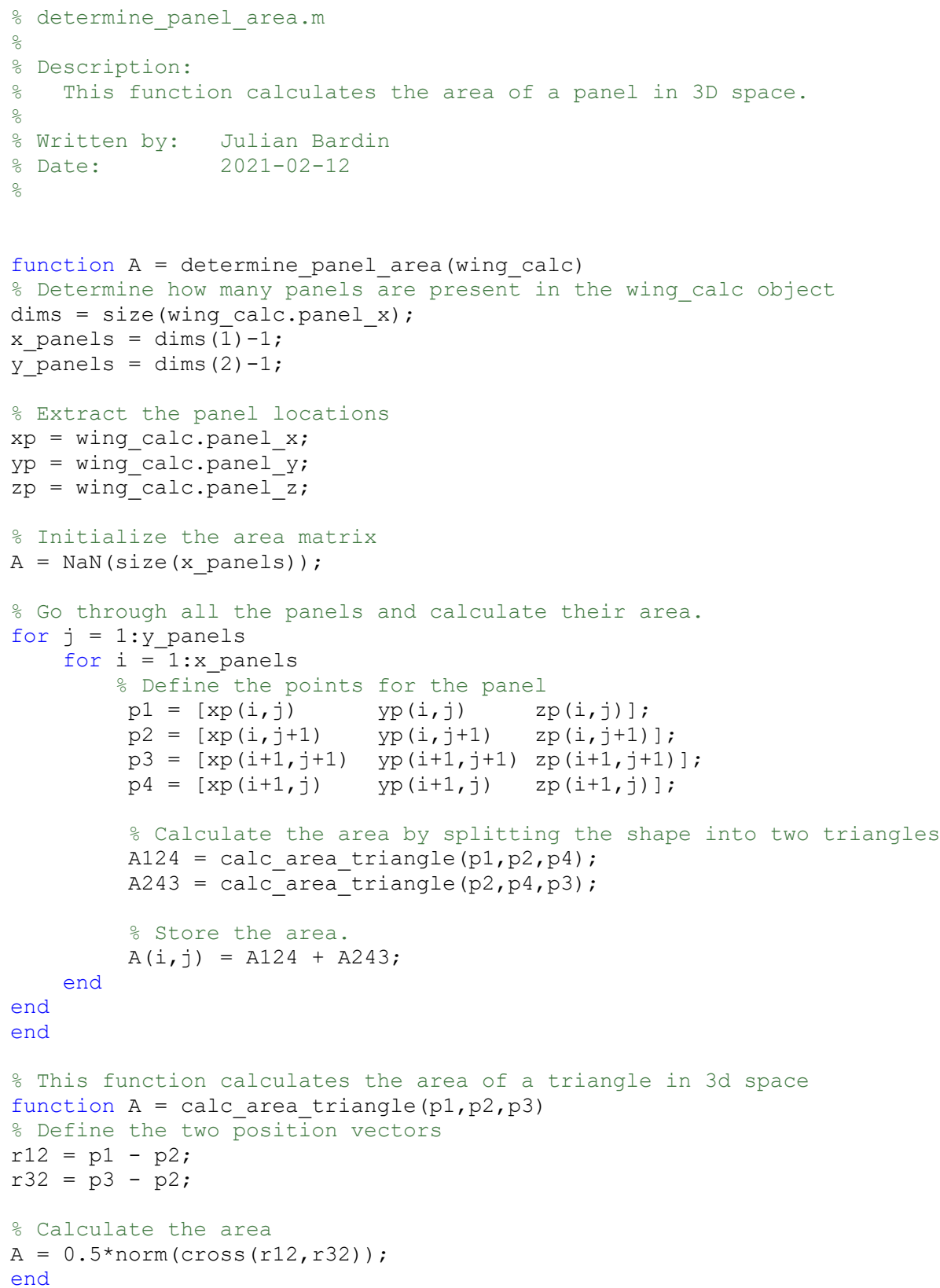




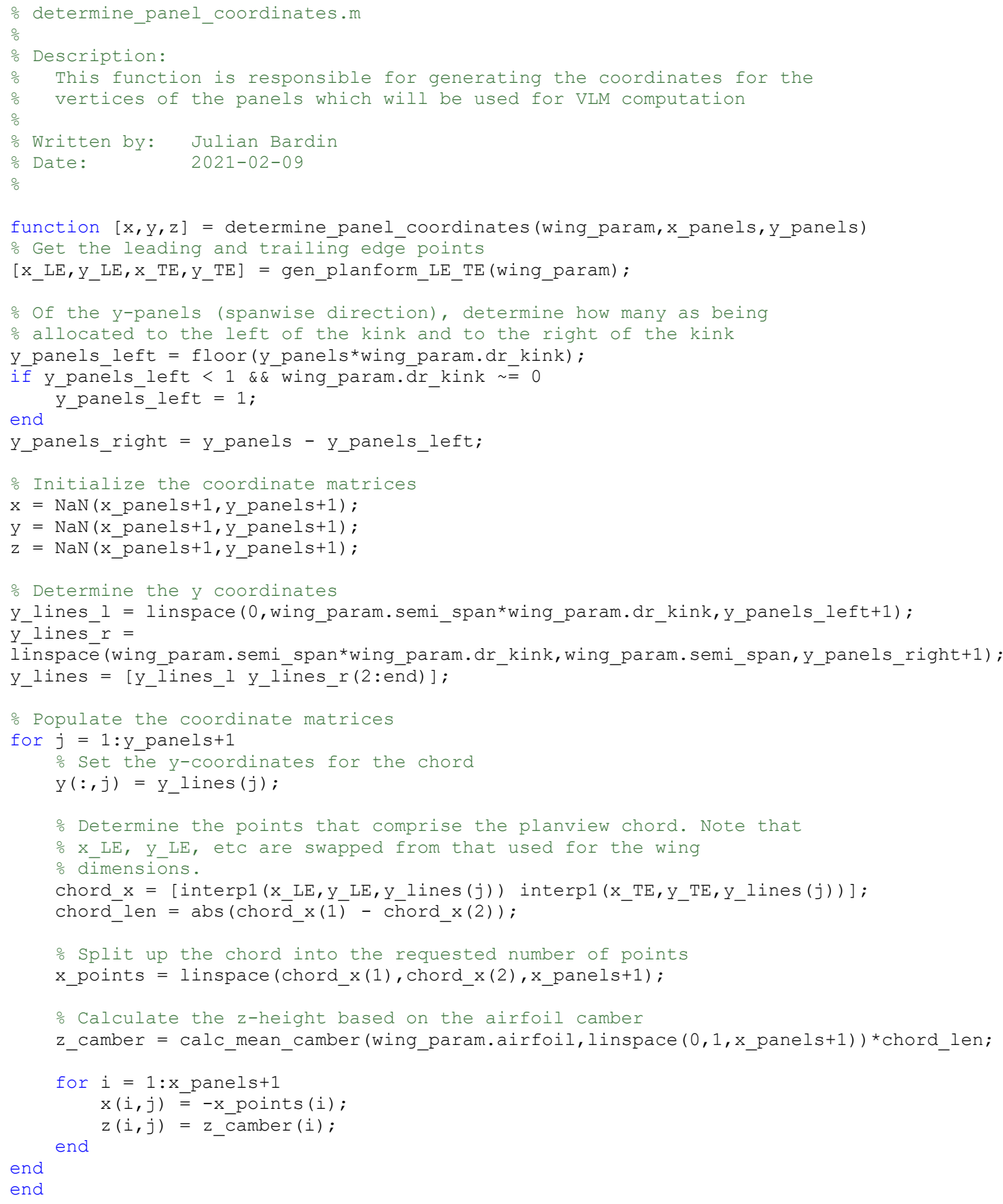




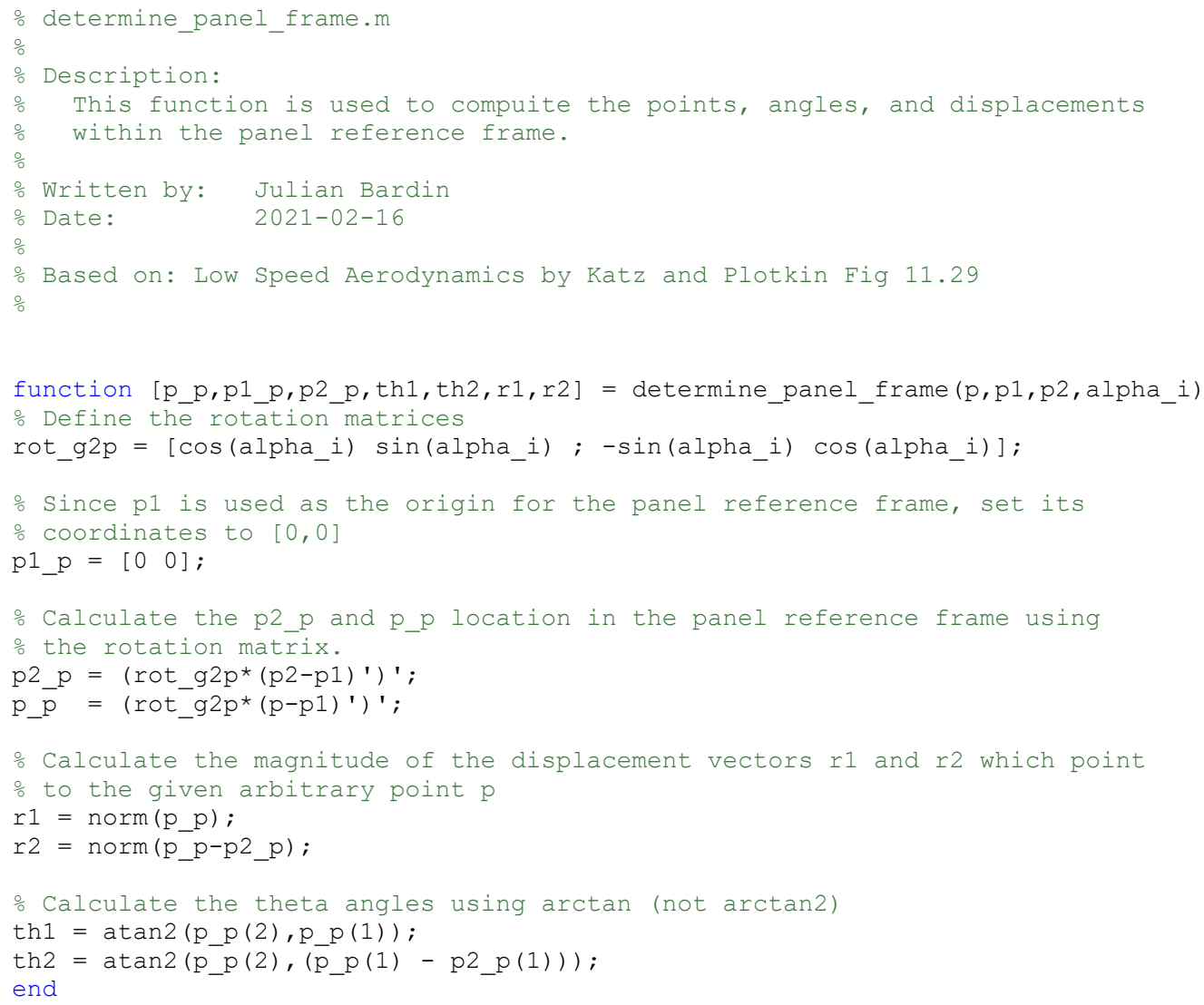




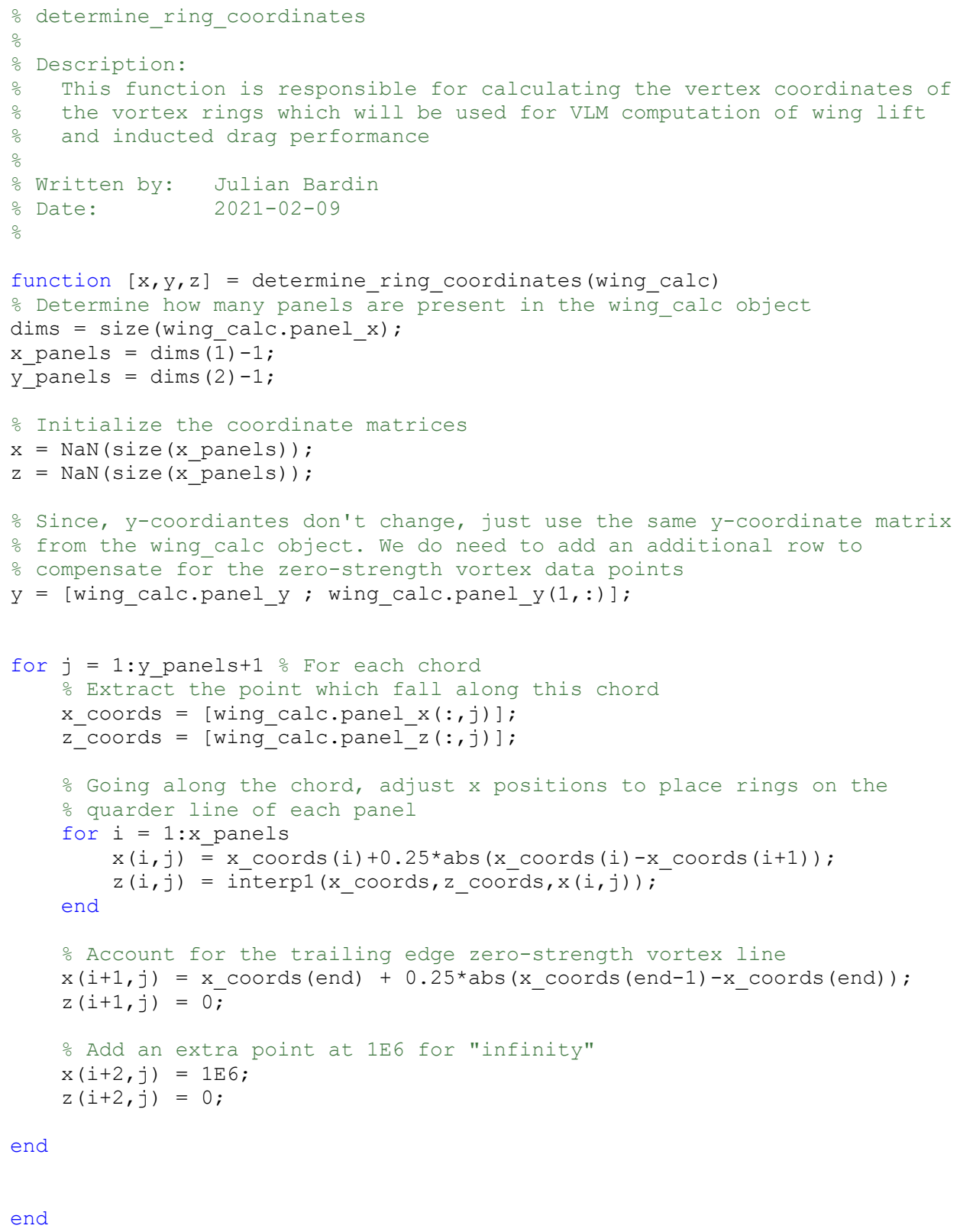




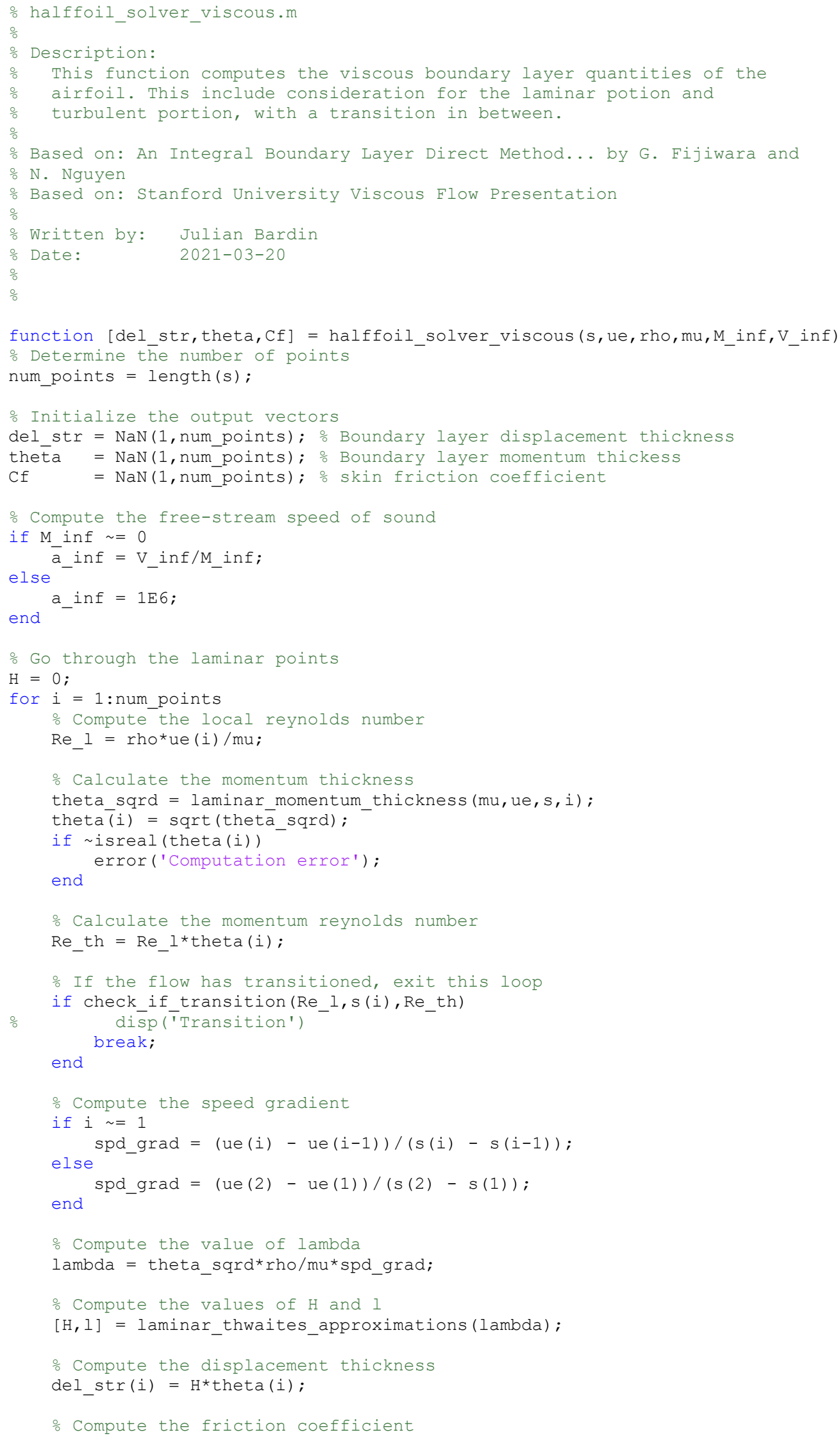




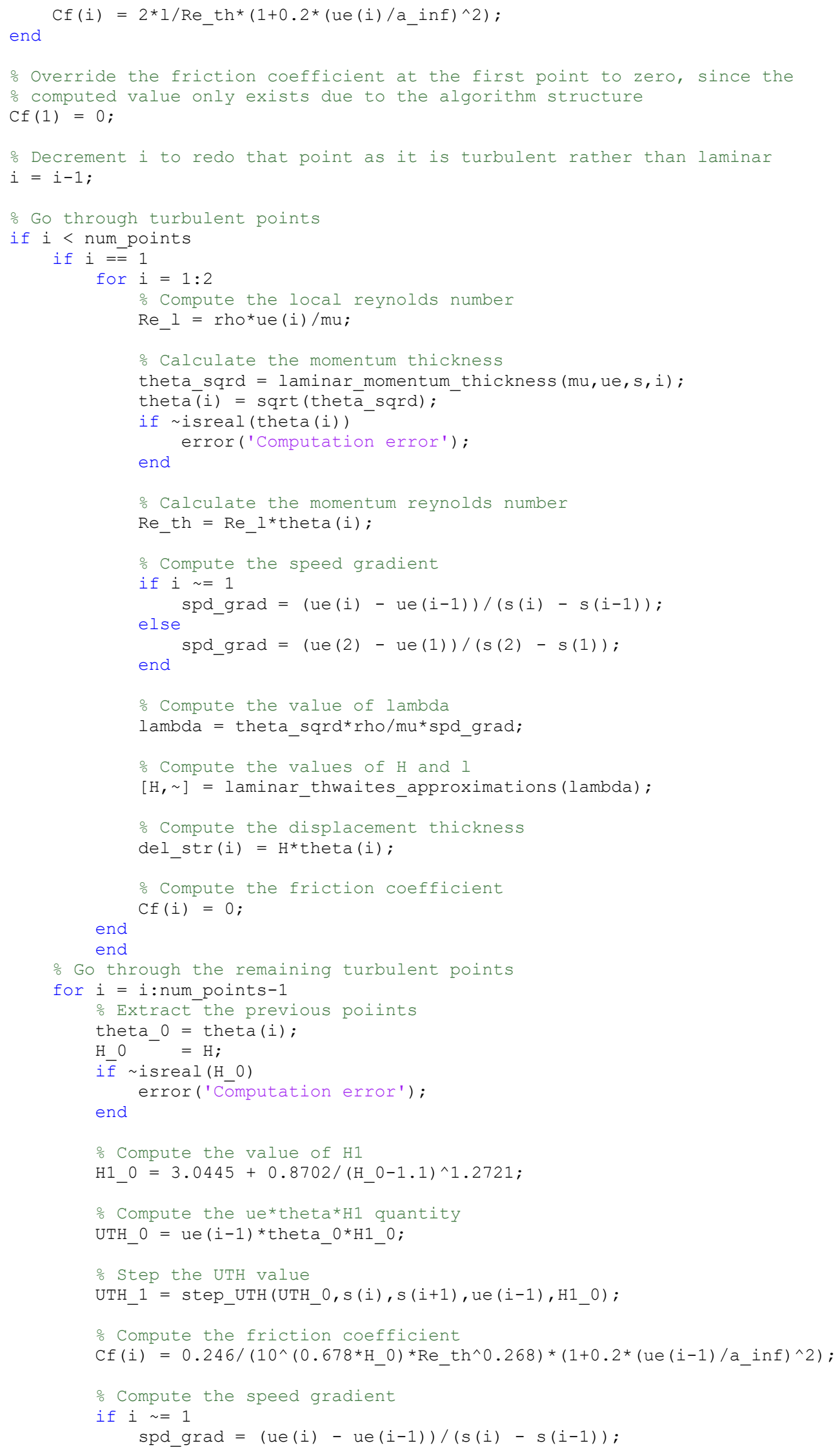




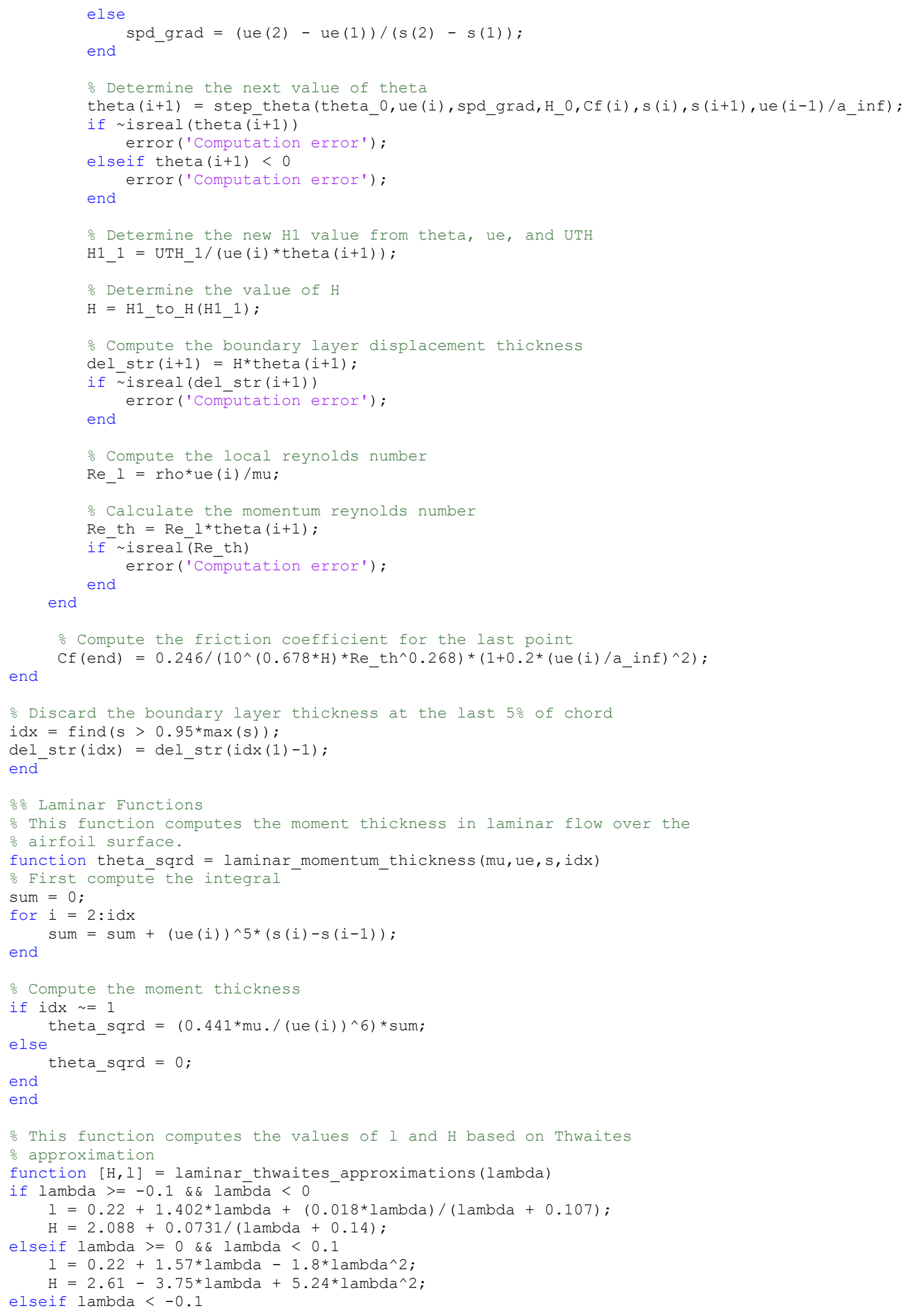




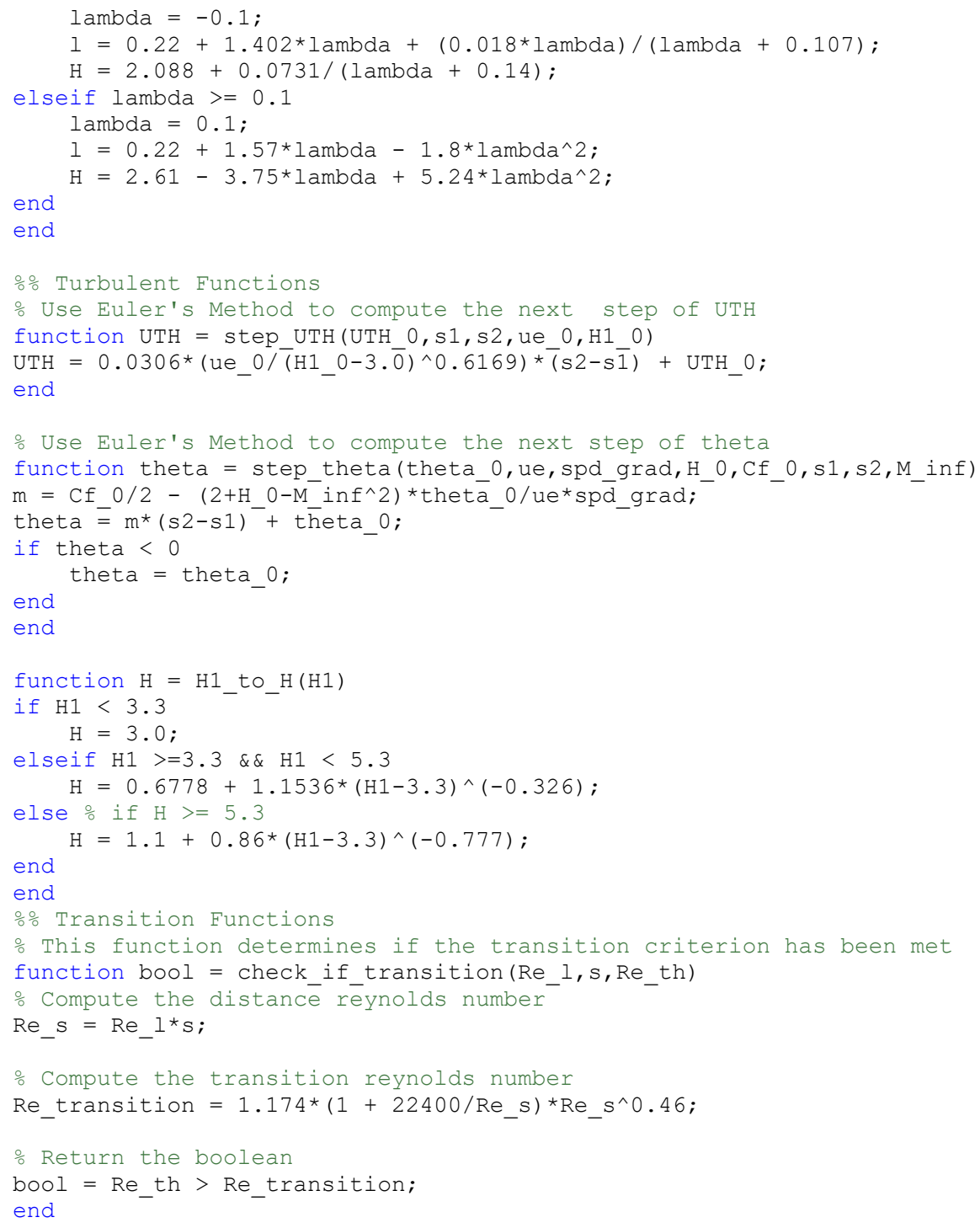




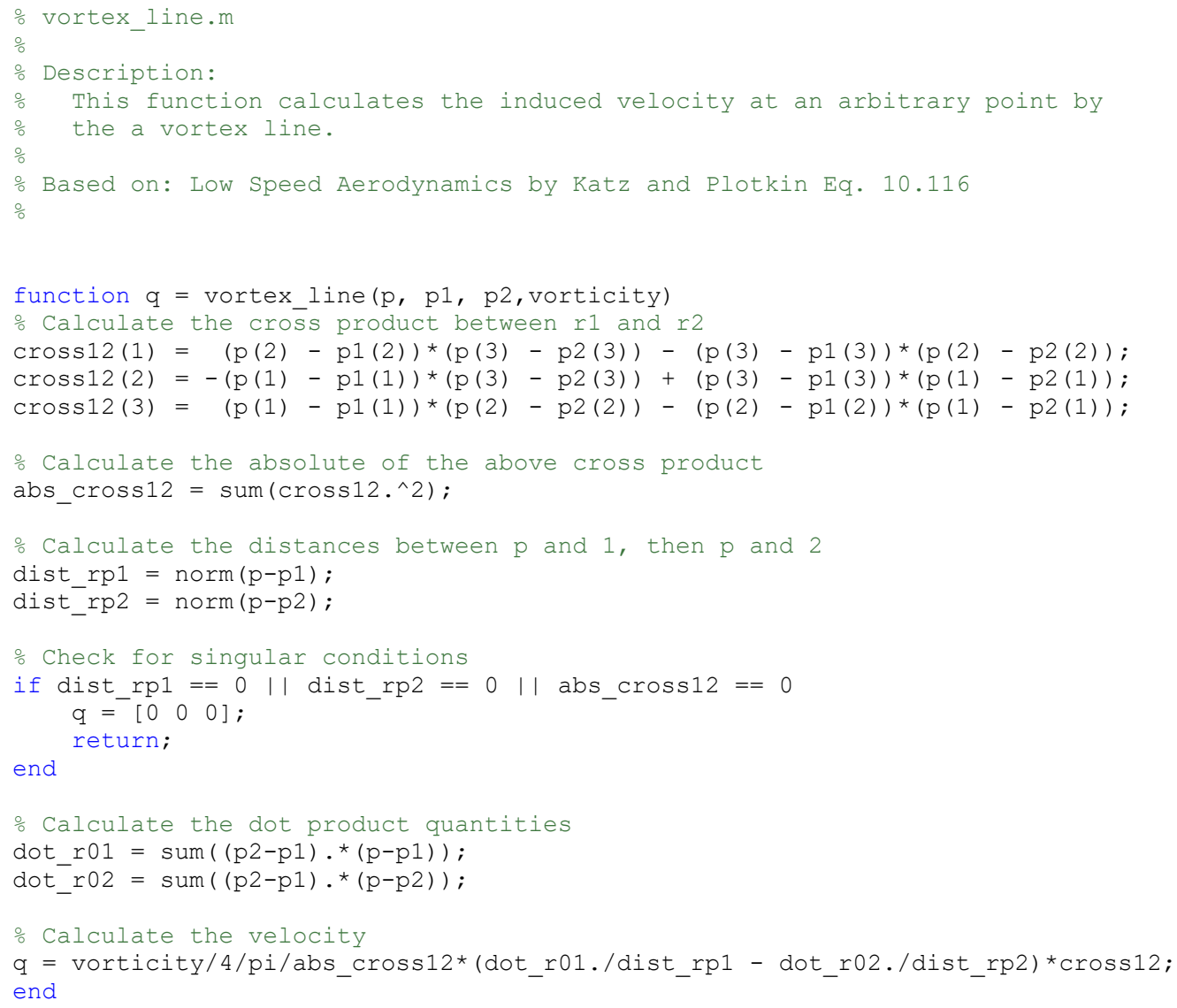




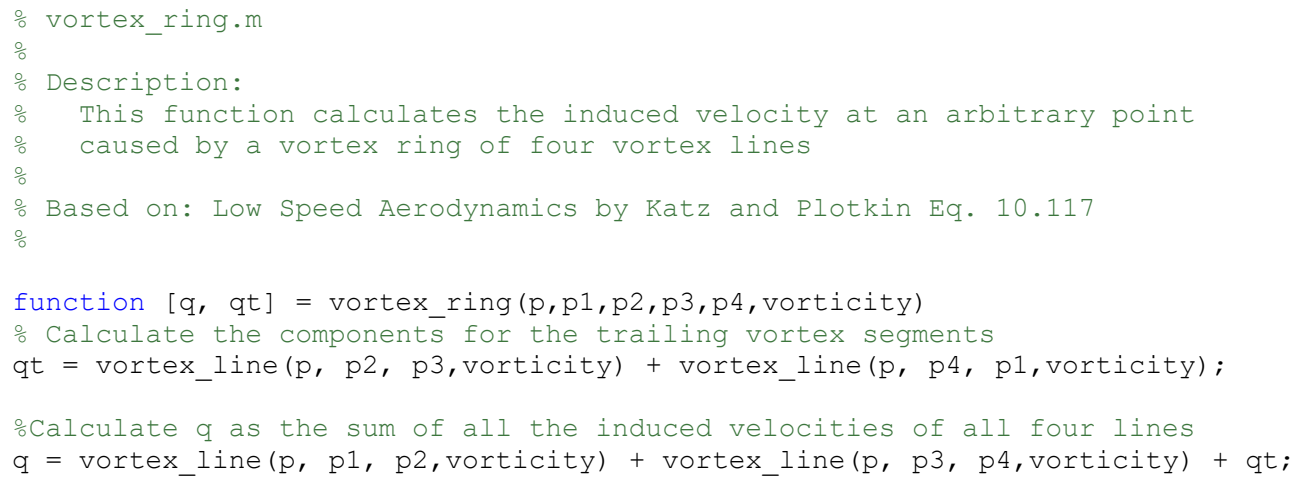




\section{Appendix F: Structural Functions}

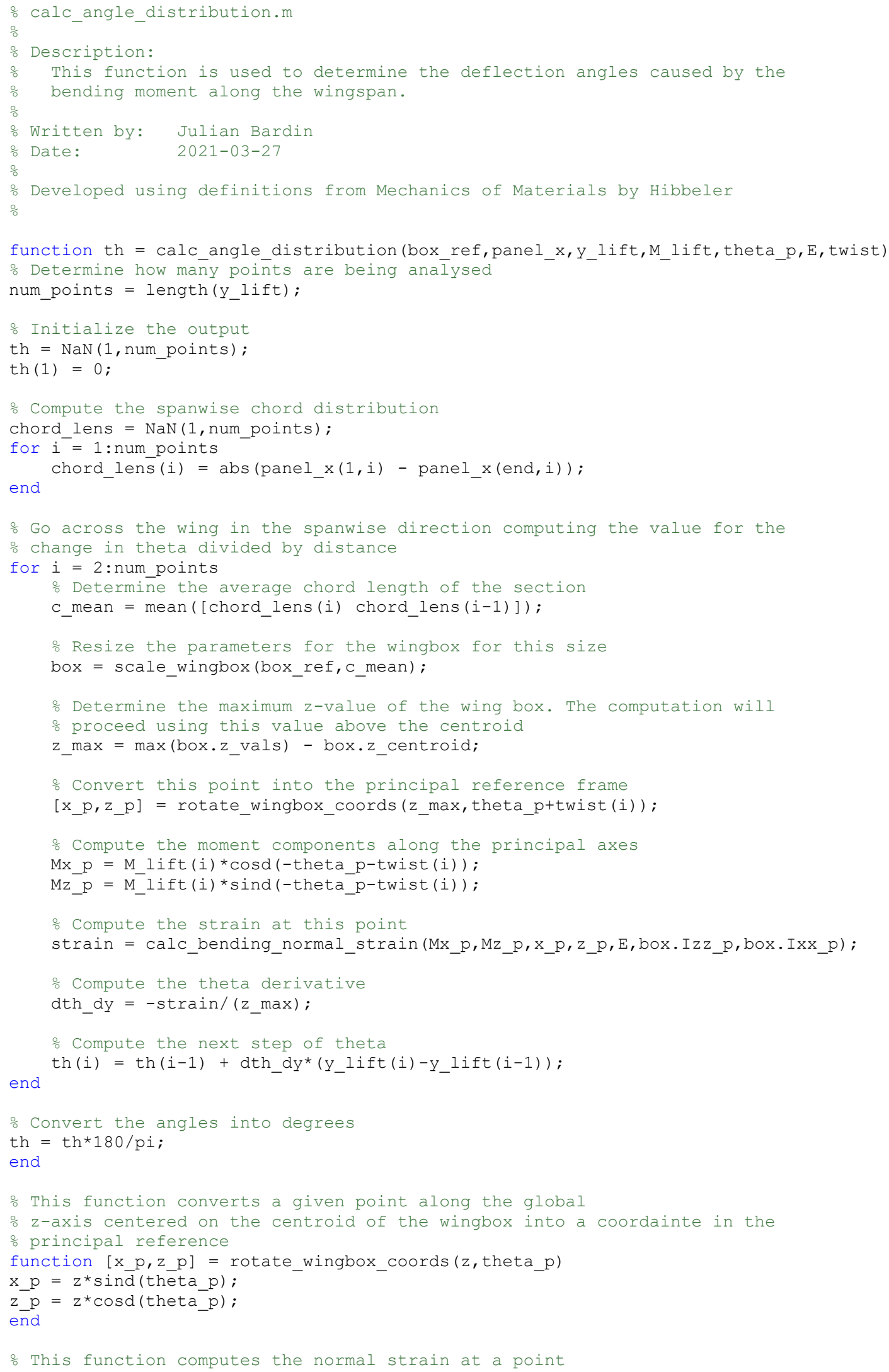


function strain = calc_bending_normal_strain $\left(M x \_p, M z \_p, x \_p, z \_p, E, I z z \_p, I x x \_p\right)$

strain $=\mathrm{Mz} \_\mathrm{p}^{\star} \mathrm{x} \_\mathrm{p} /\left(\mathrm{E}^{\star} \mathrm{I} \bar{z} \mathrm{z} \_\mathrm{p}\right)-\overline{\mathrm{M}} \mathrm{x} \_\mathrm{p}^{*} \mathrm{z} \_\overline{\mathrm{p}} /\left(\mathrm{E}^{*} \mathrm{Ixx} \_\mathrm{p}\right)$;

end 


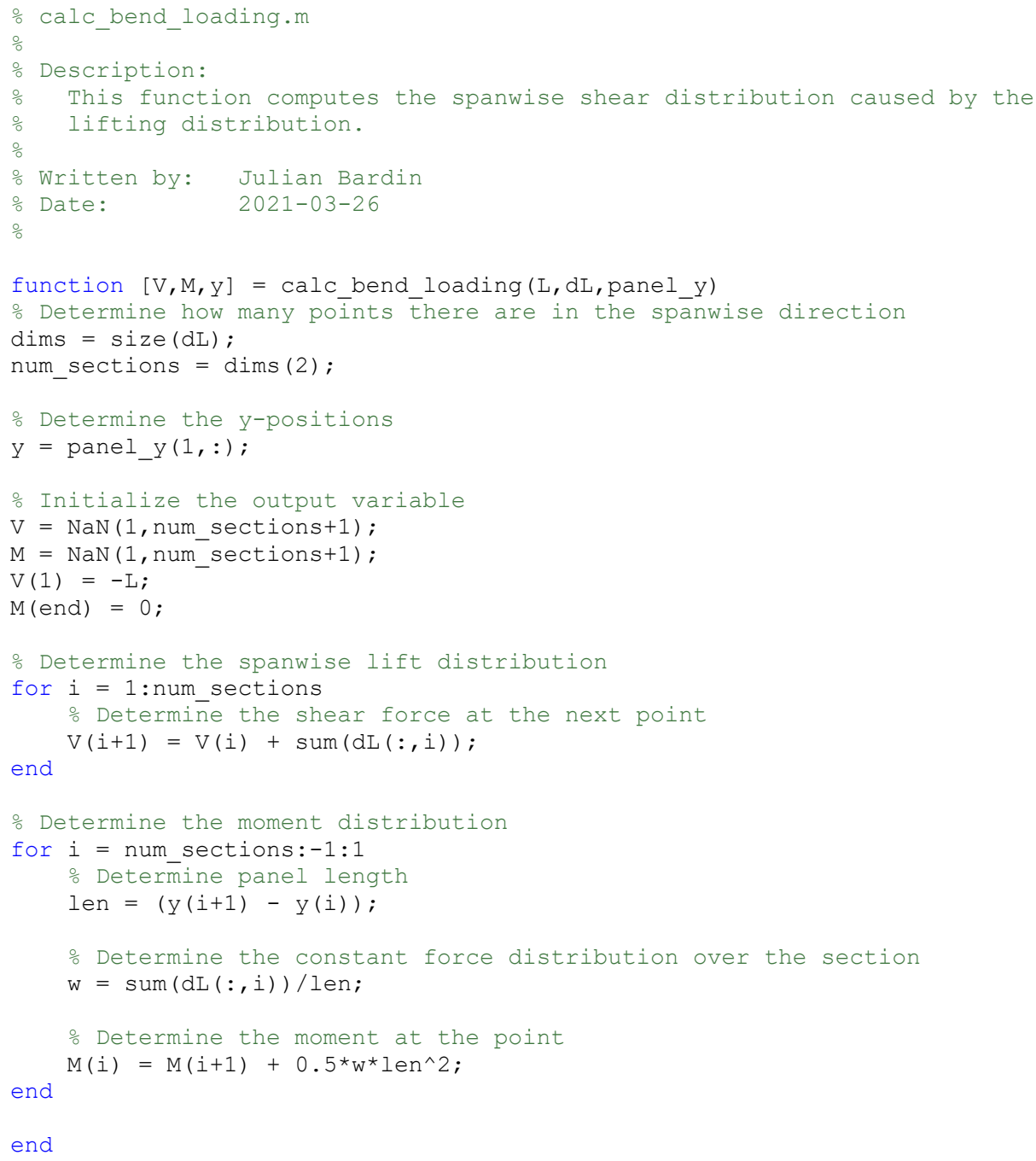




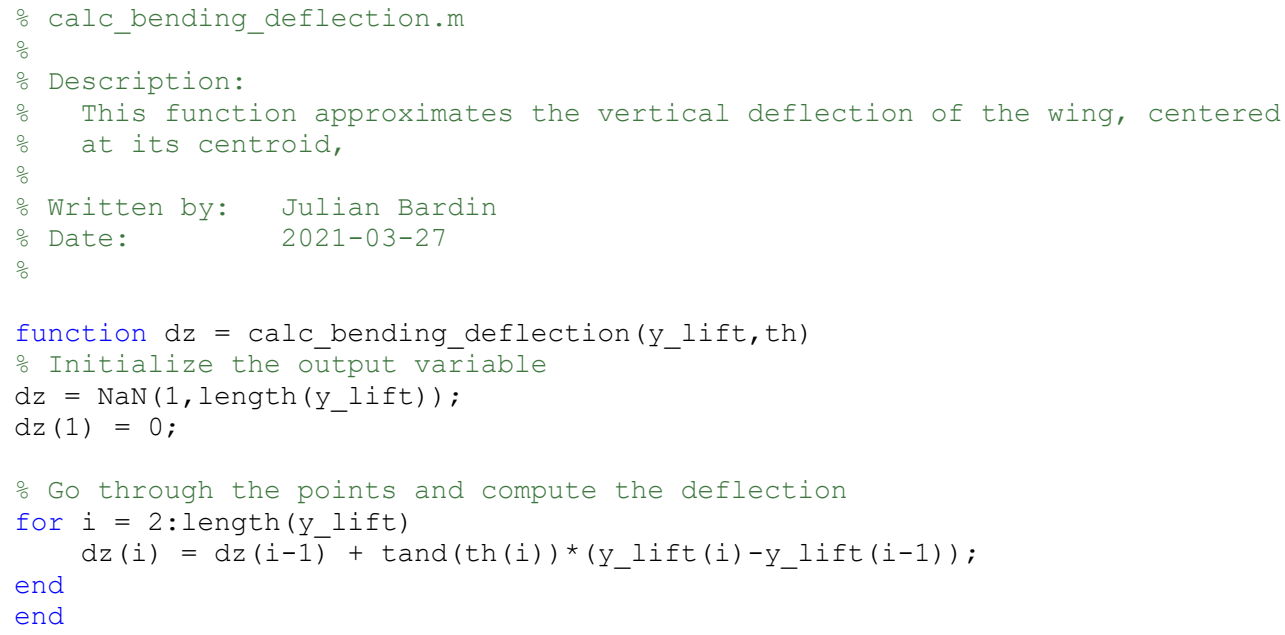




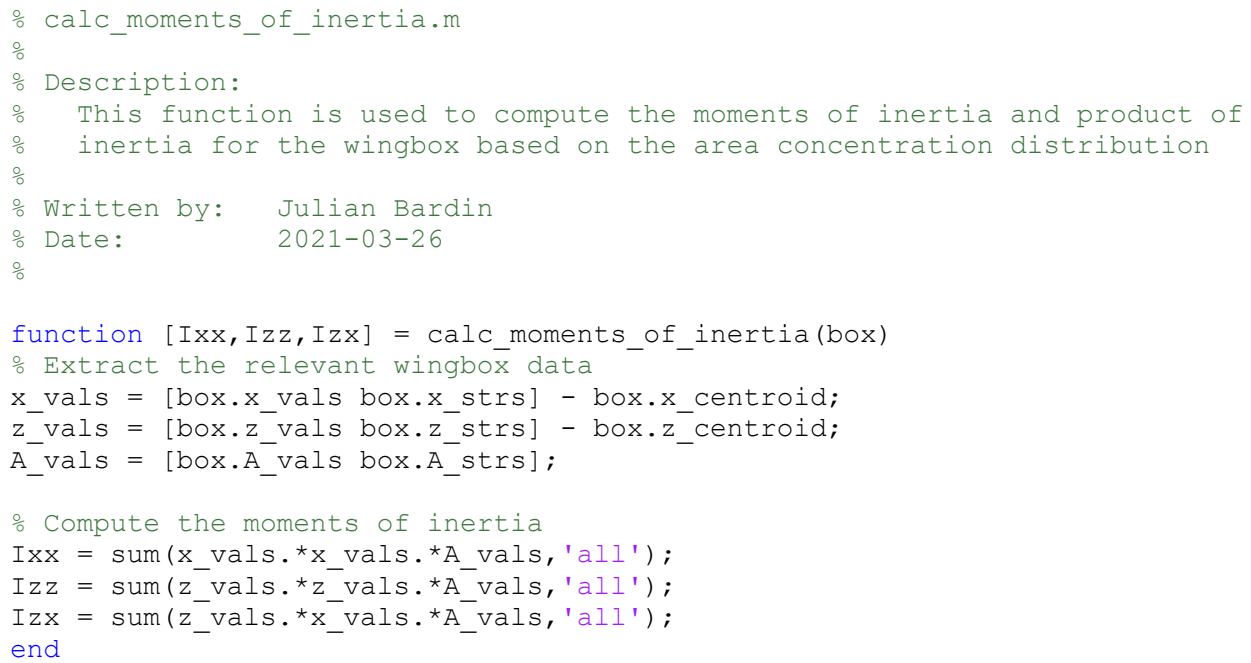




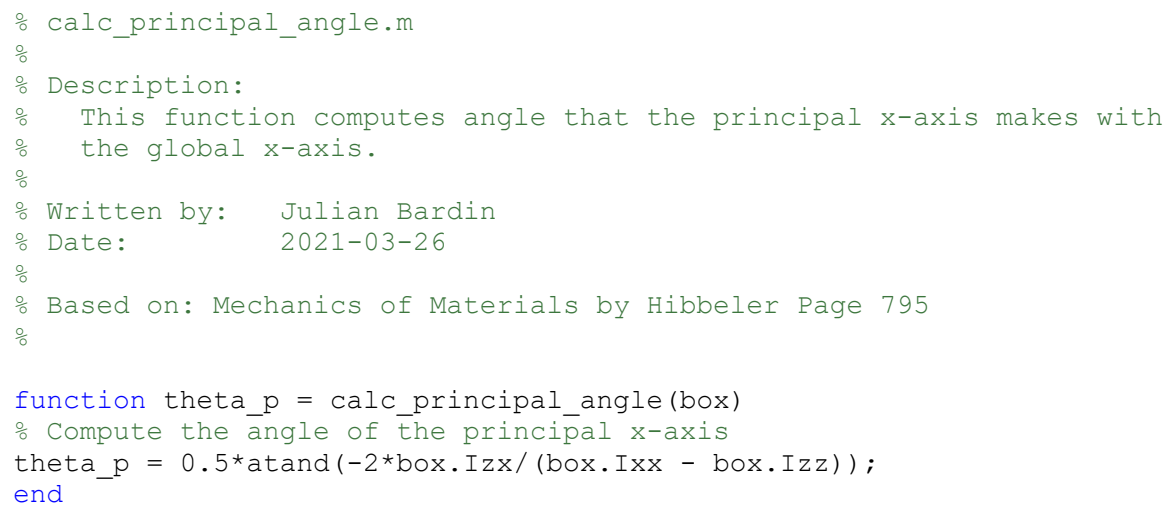




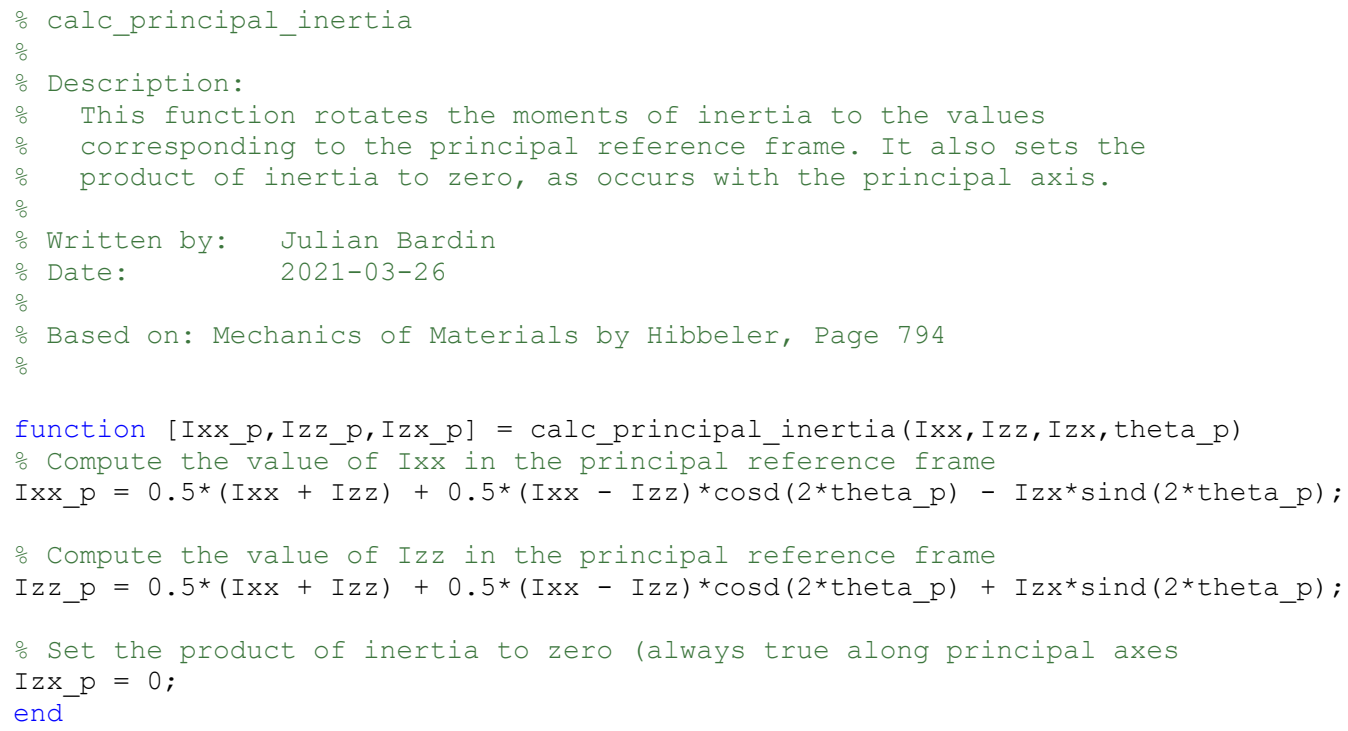




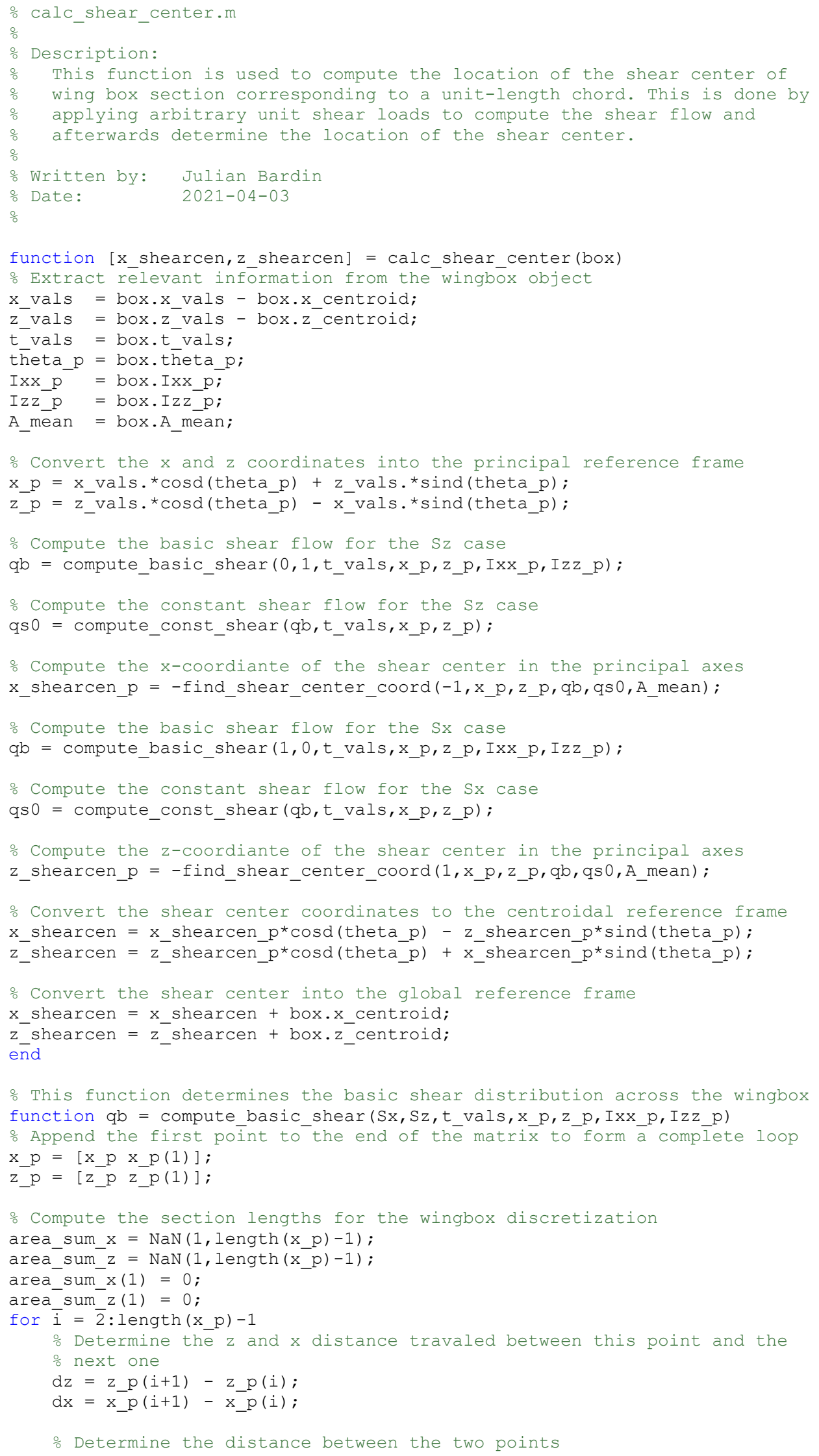




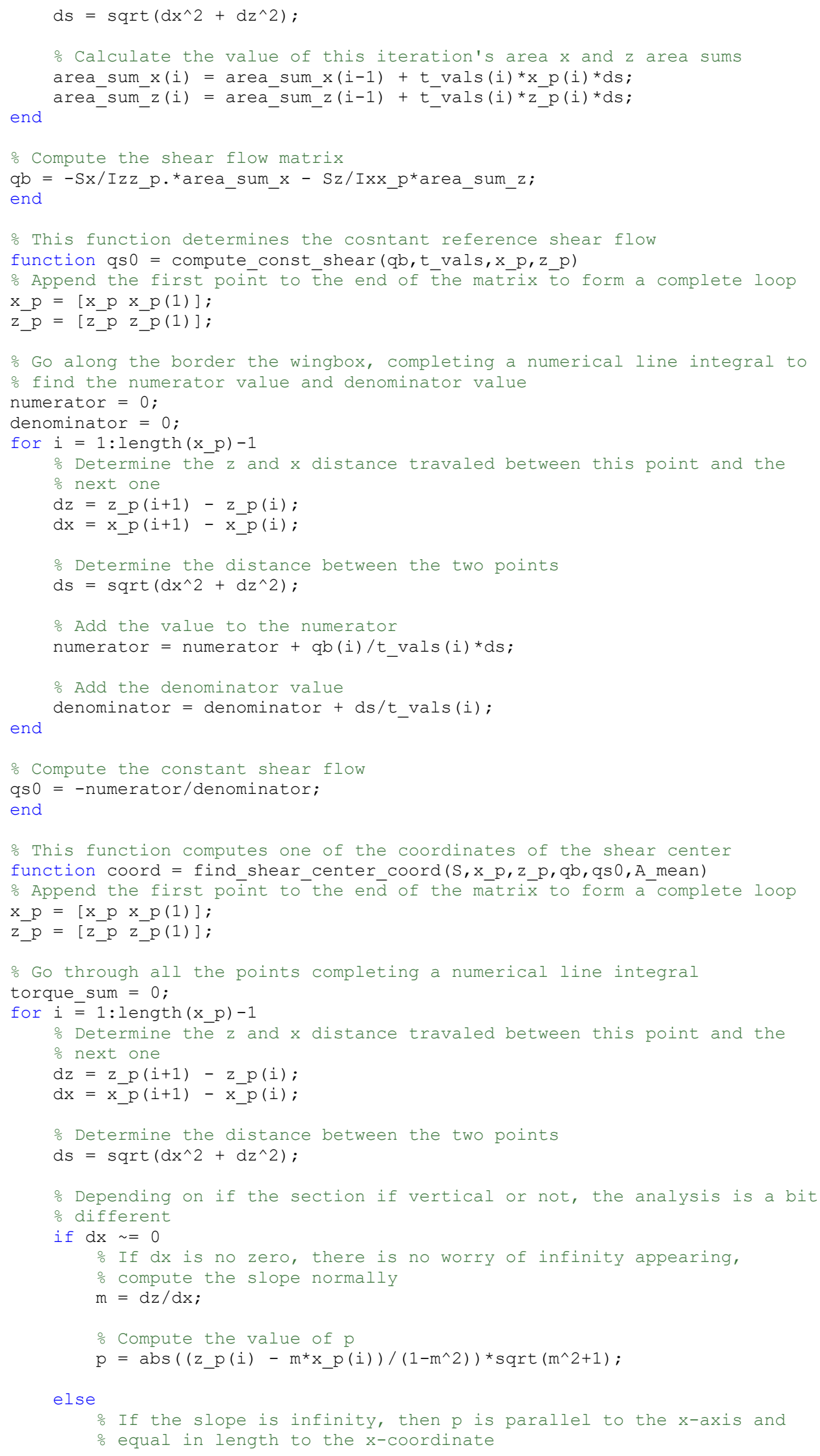




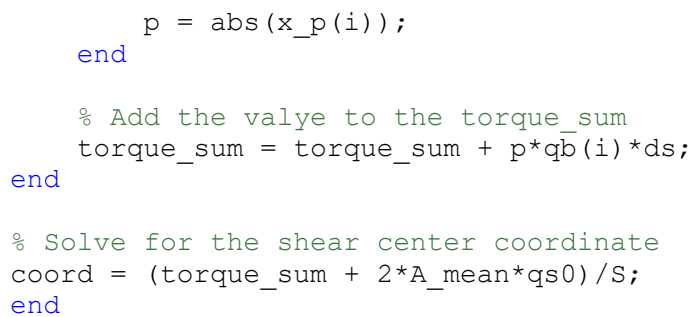




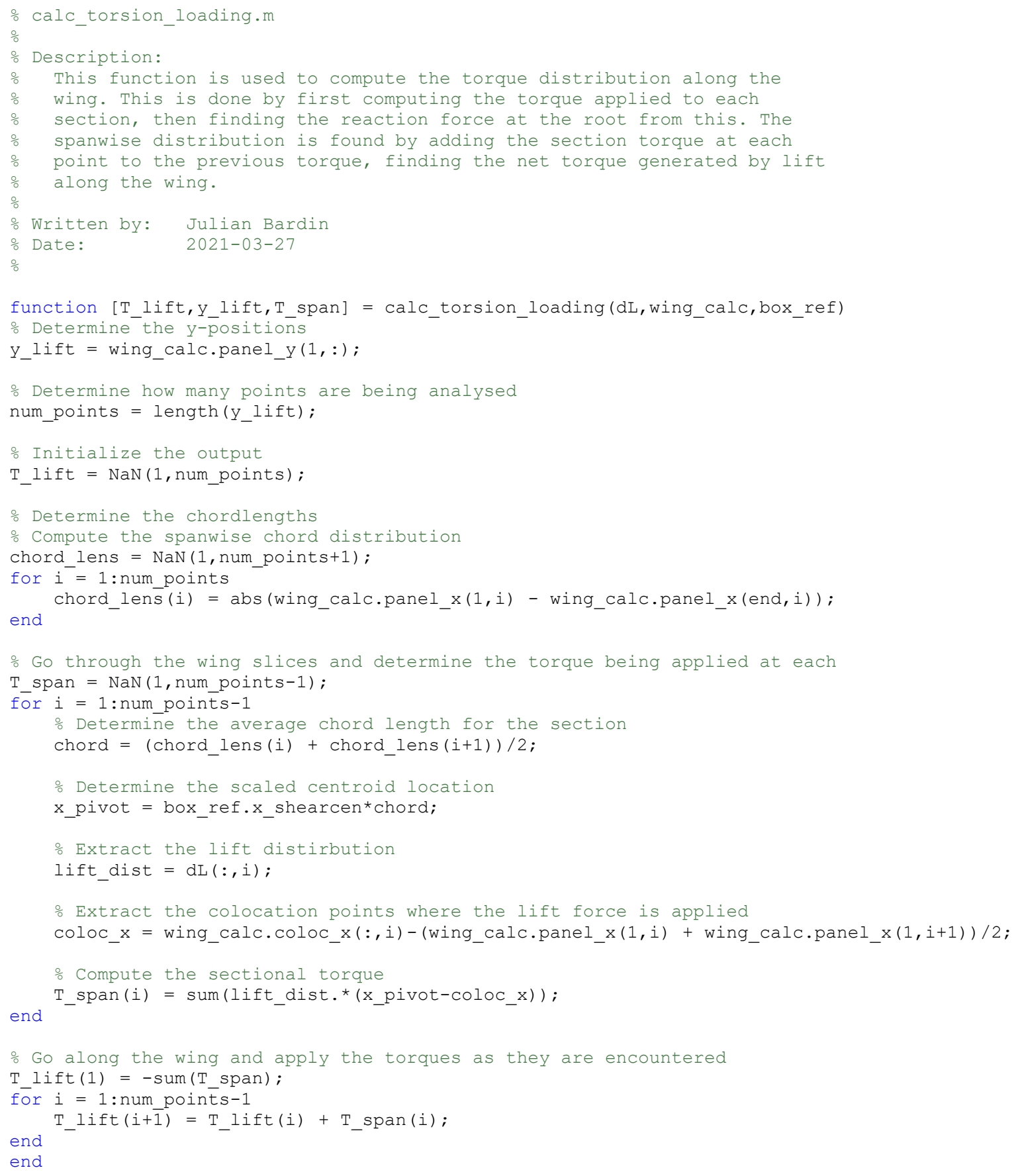




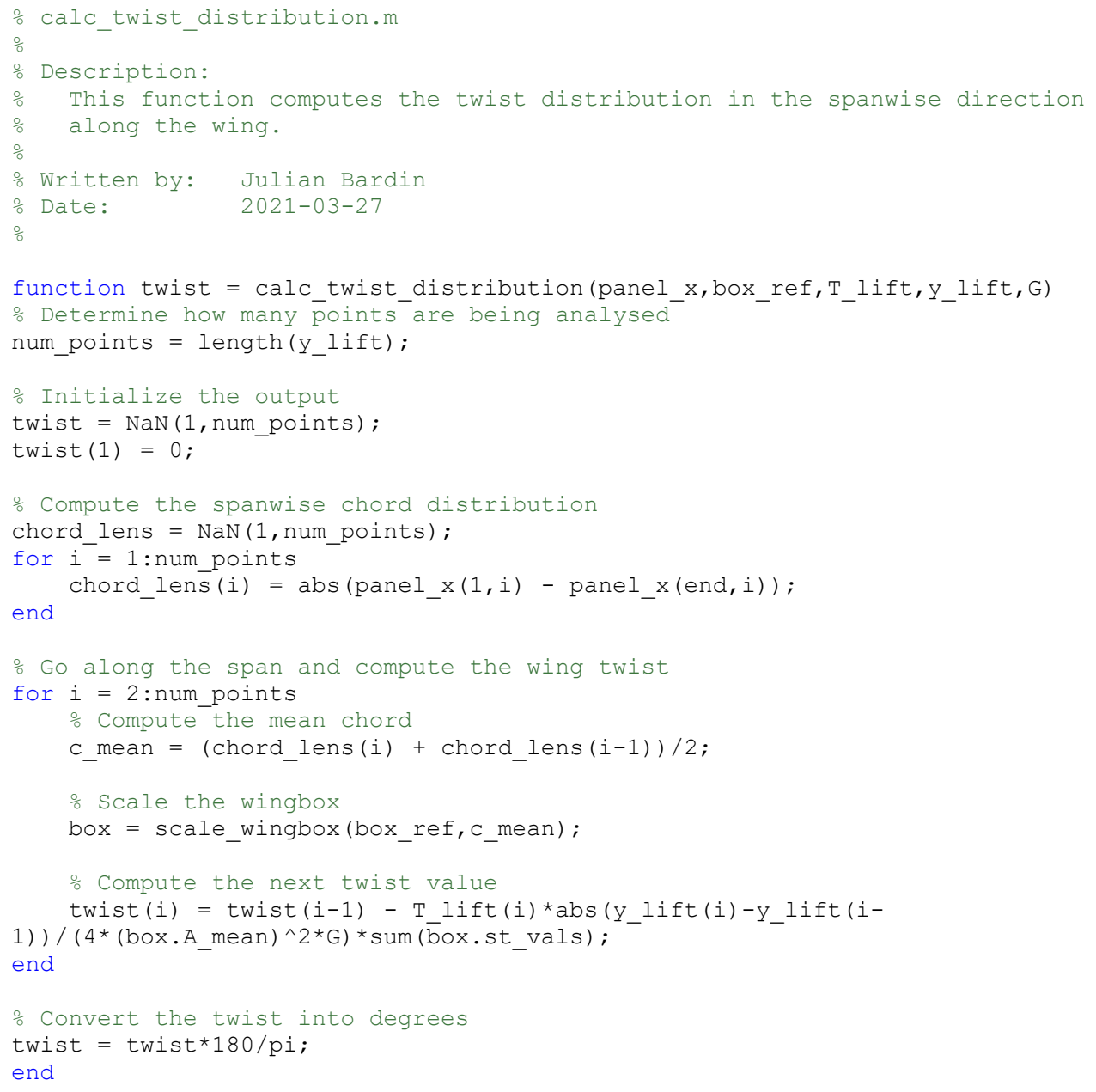




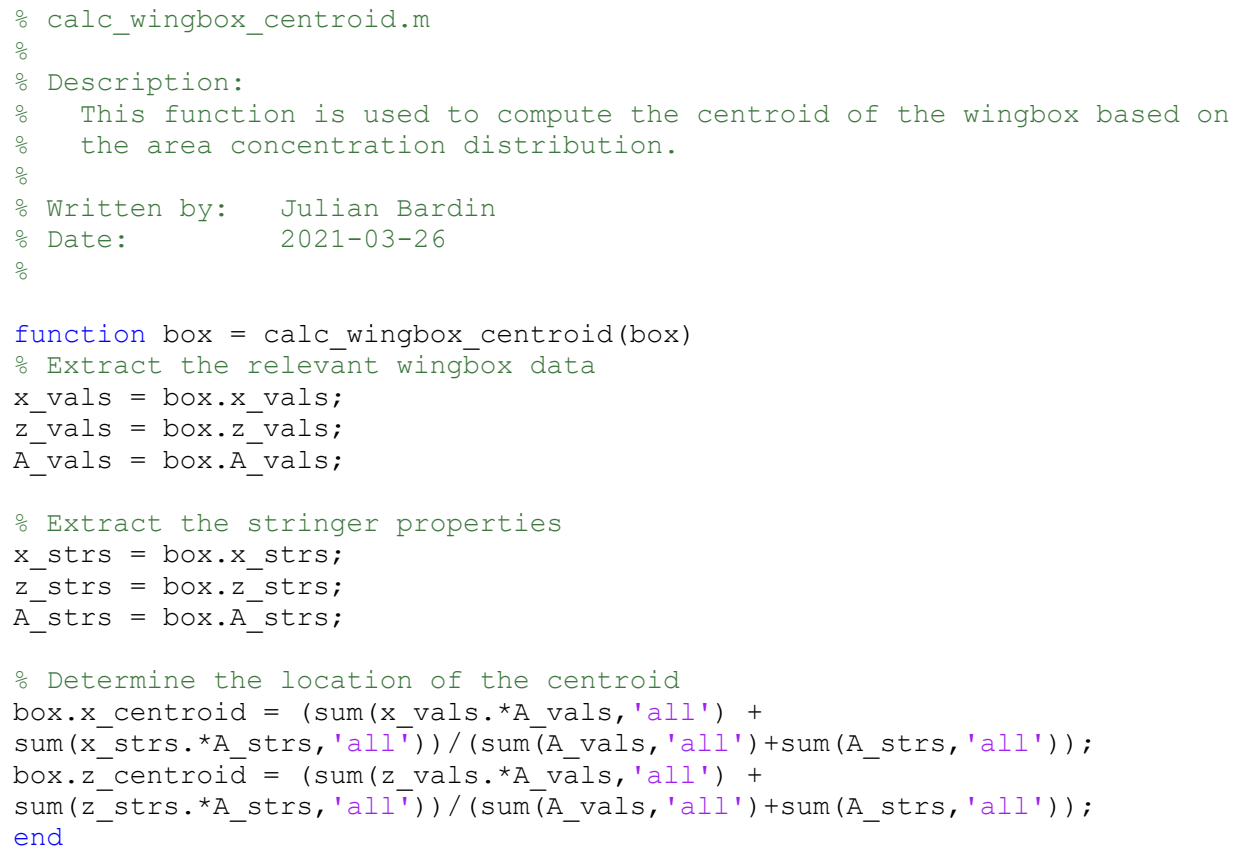




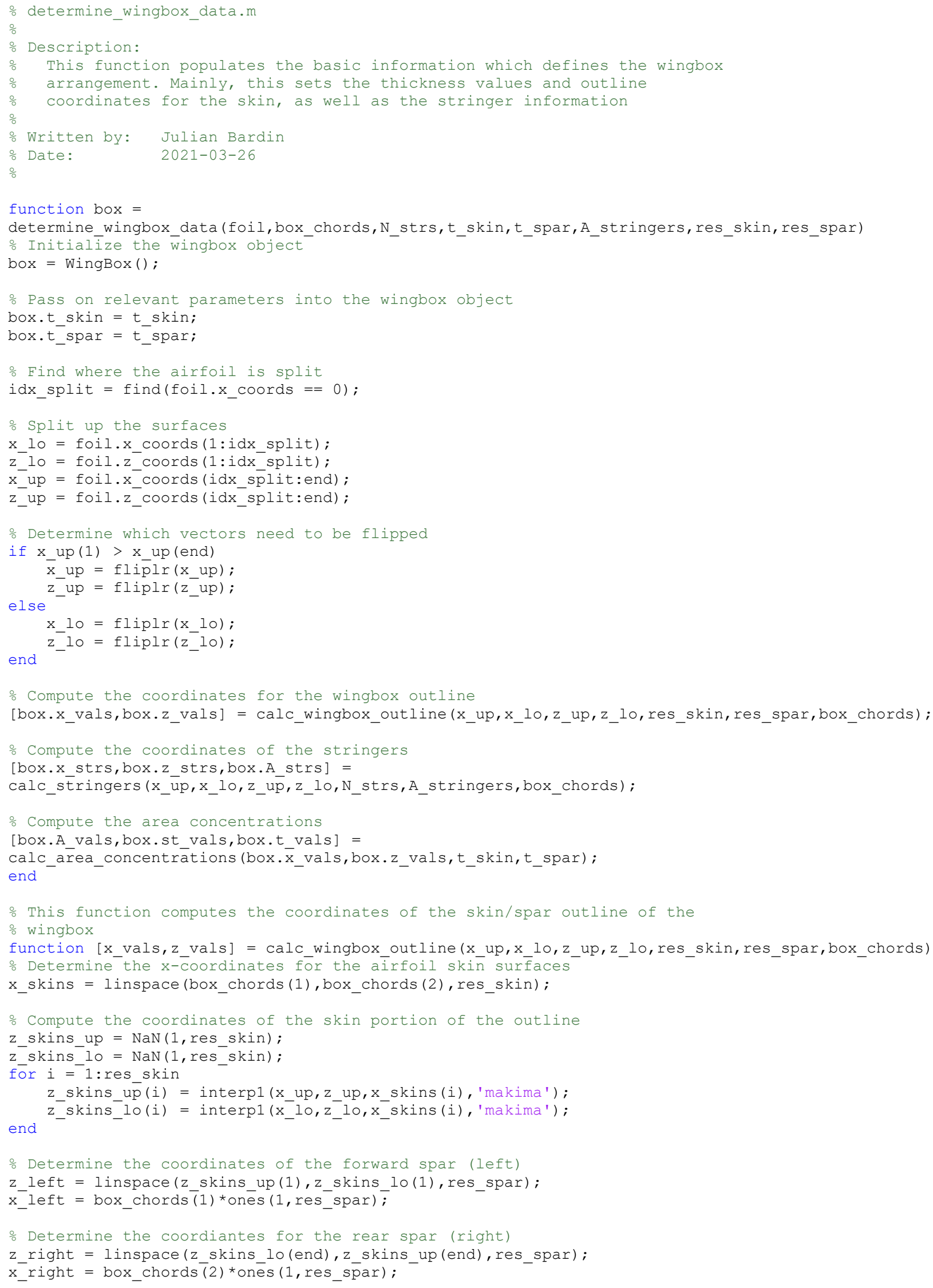




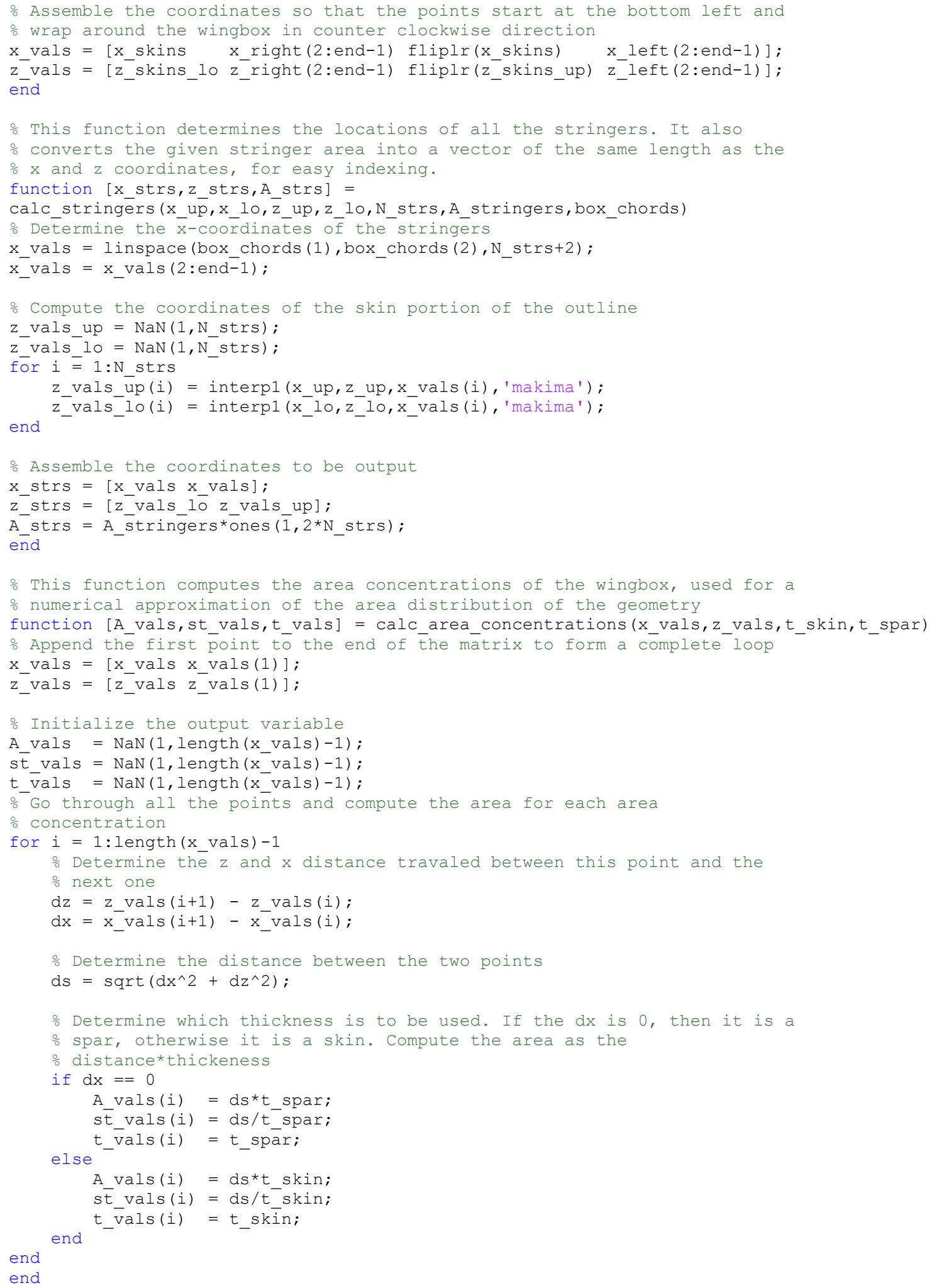




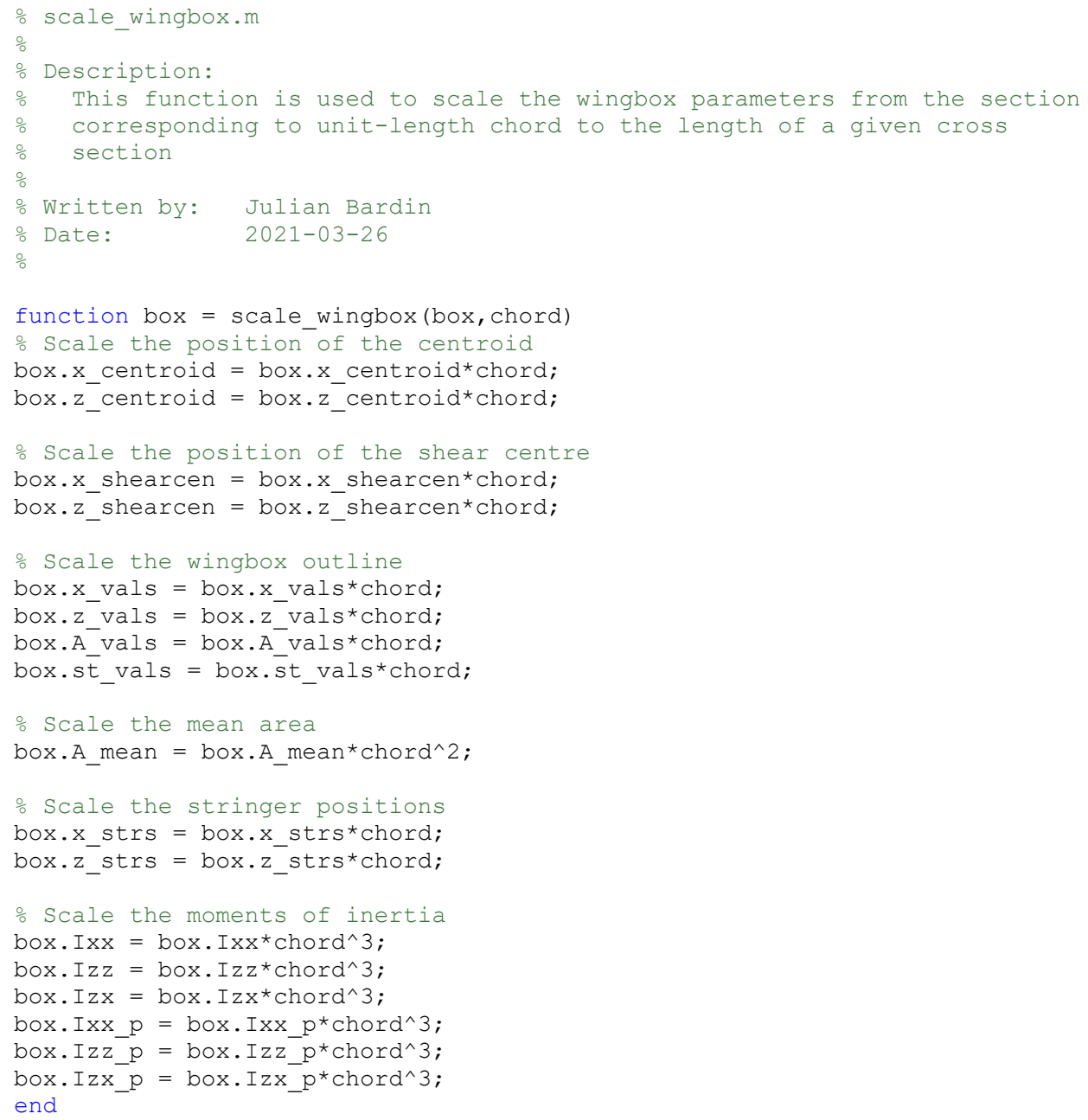




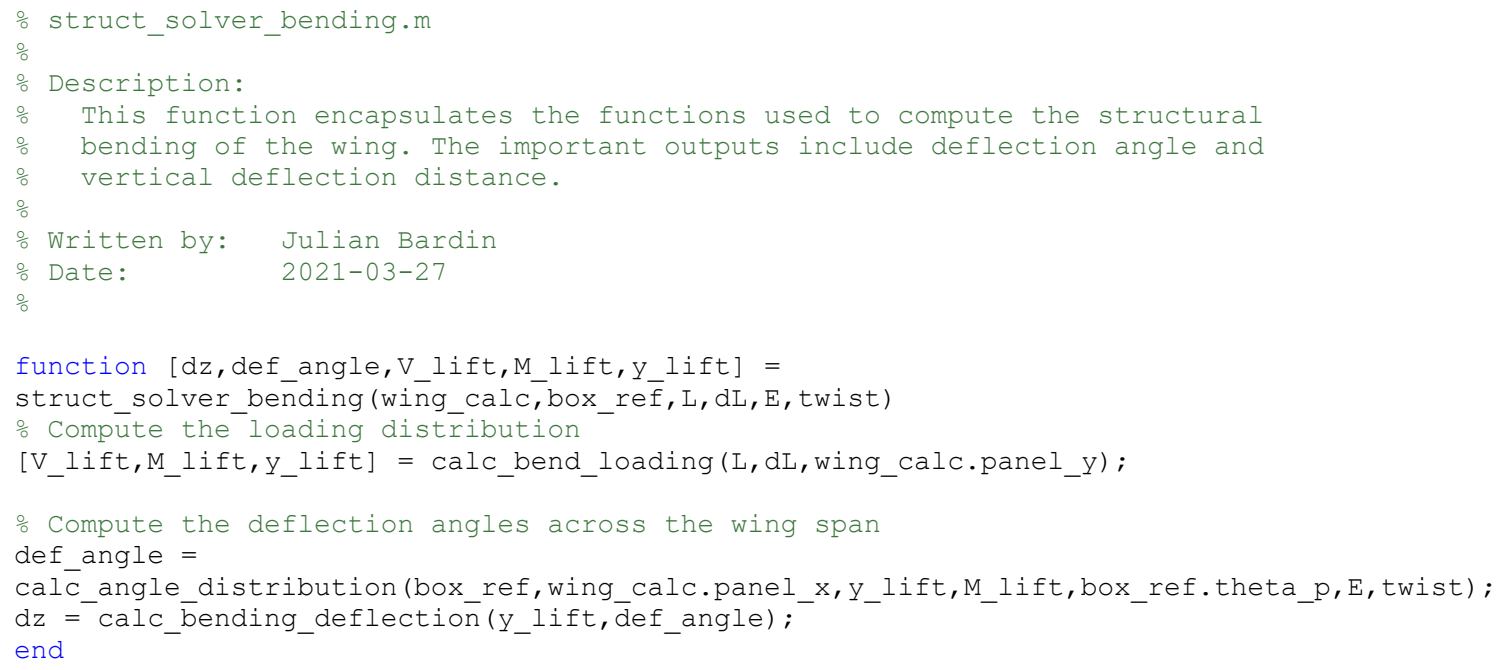




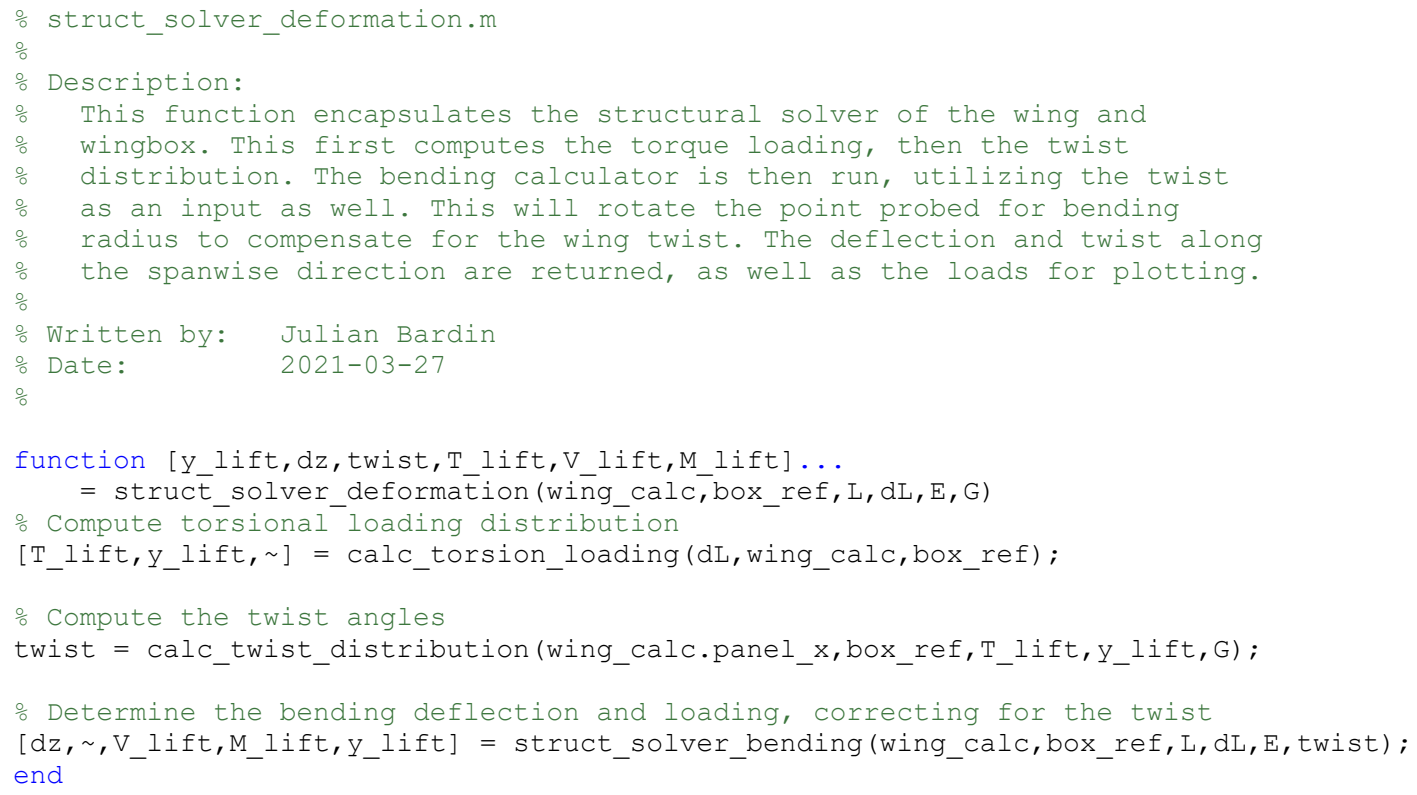




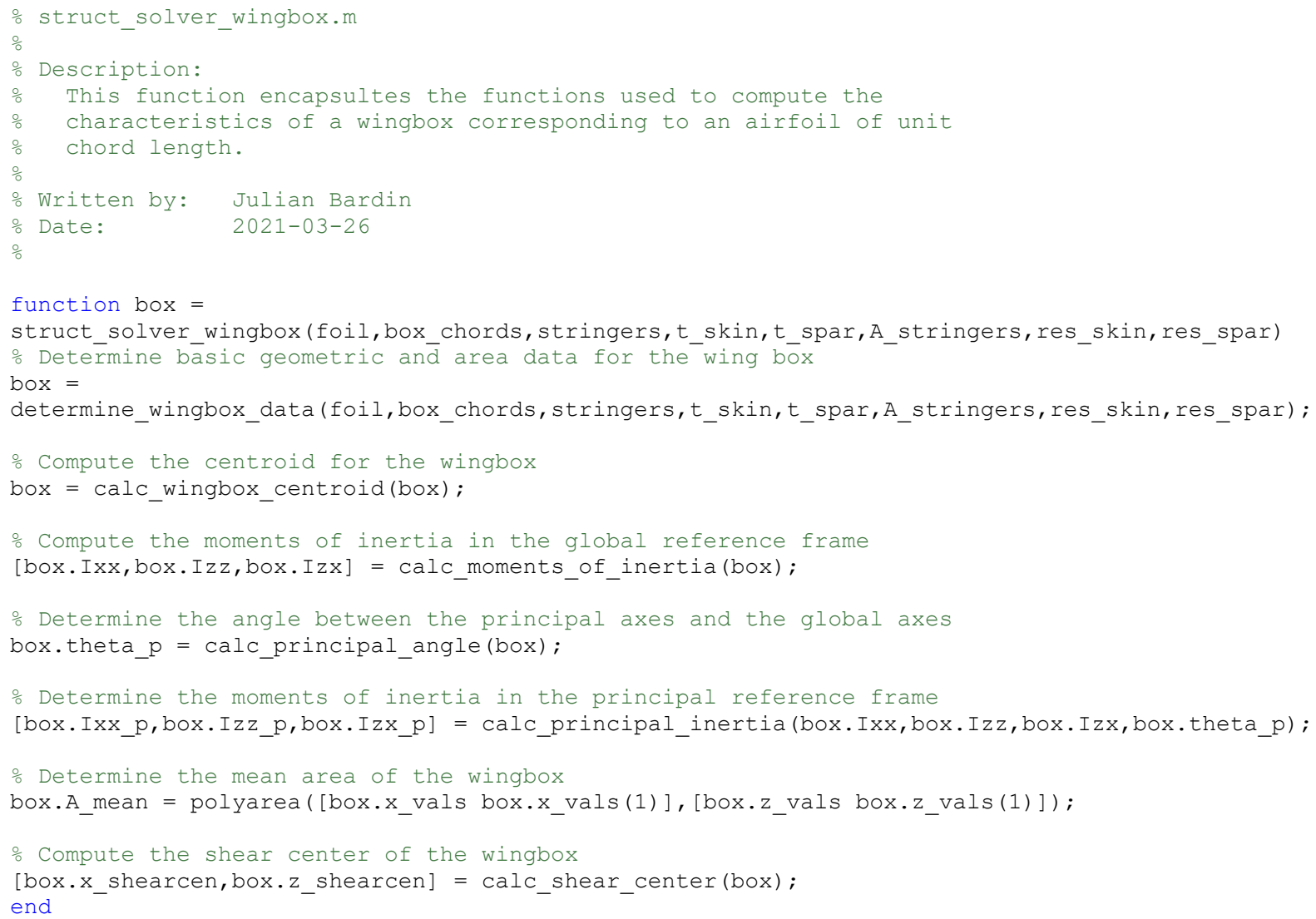




\section{Appendix G: Utility Functions}

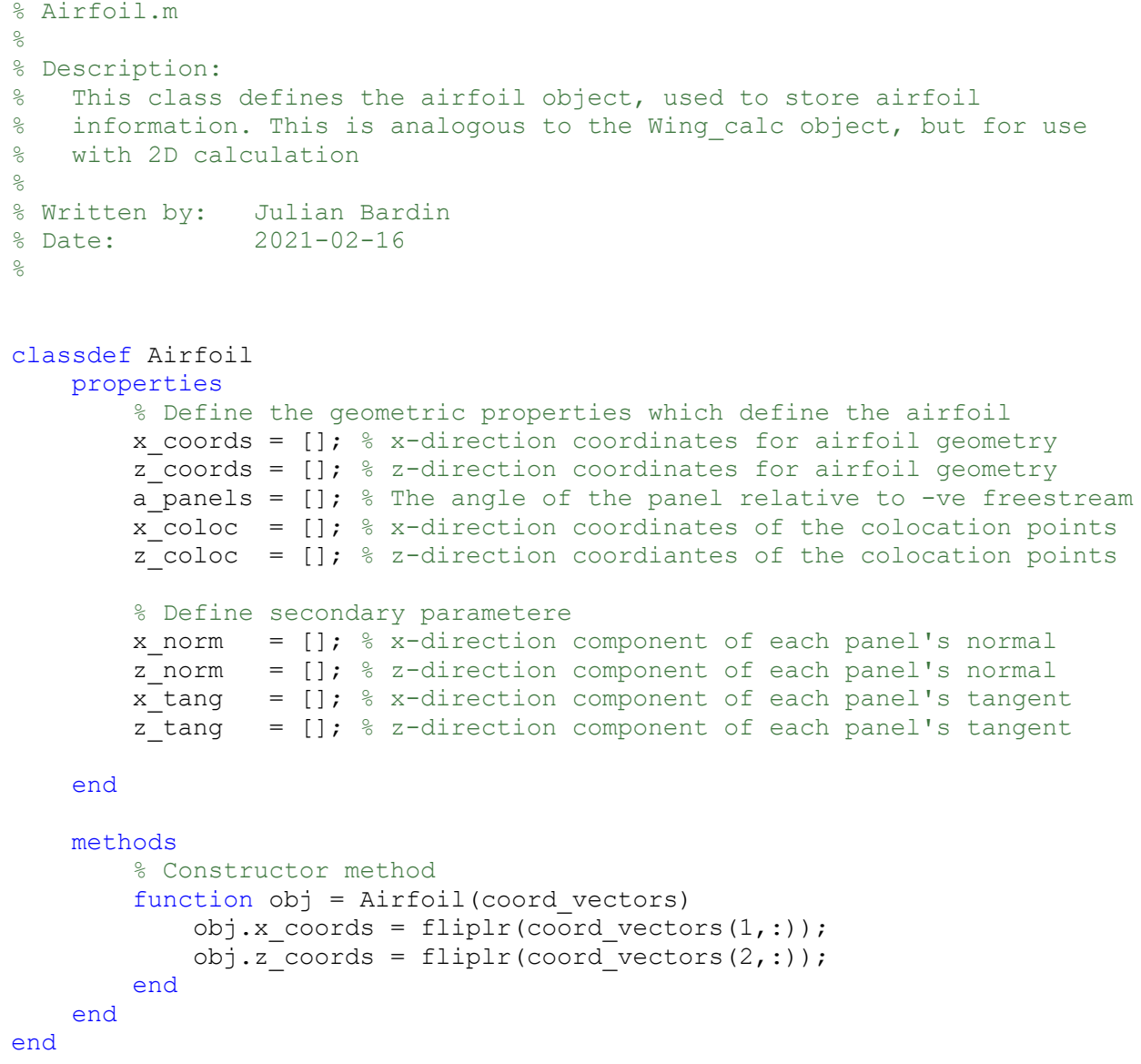




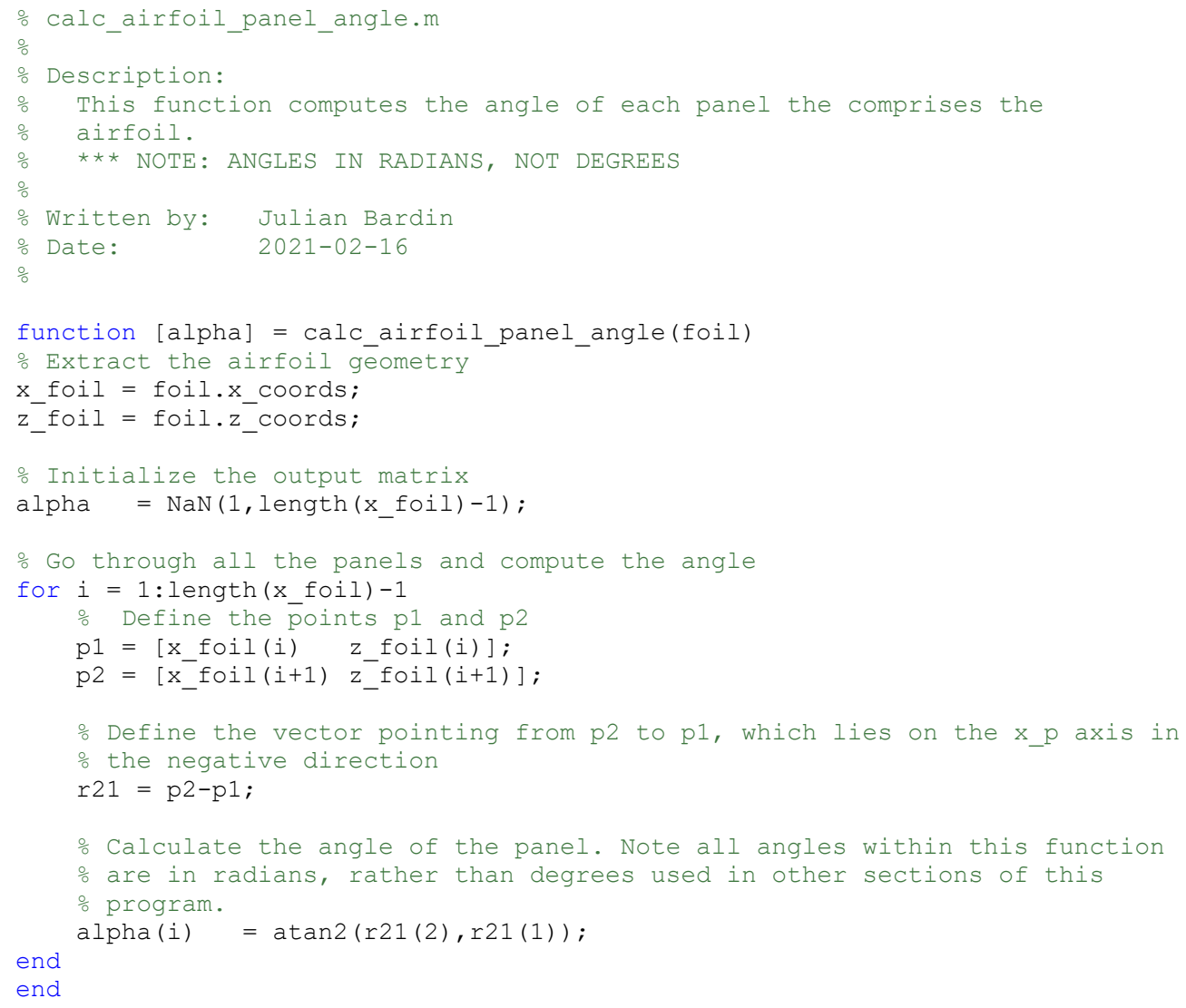




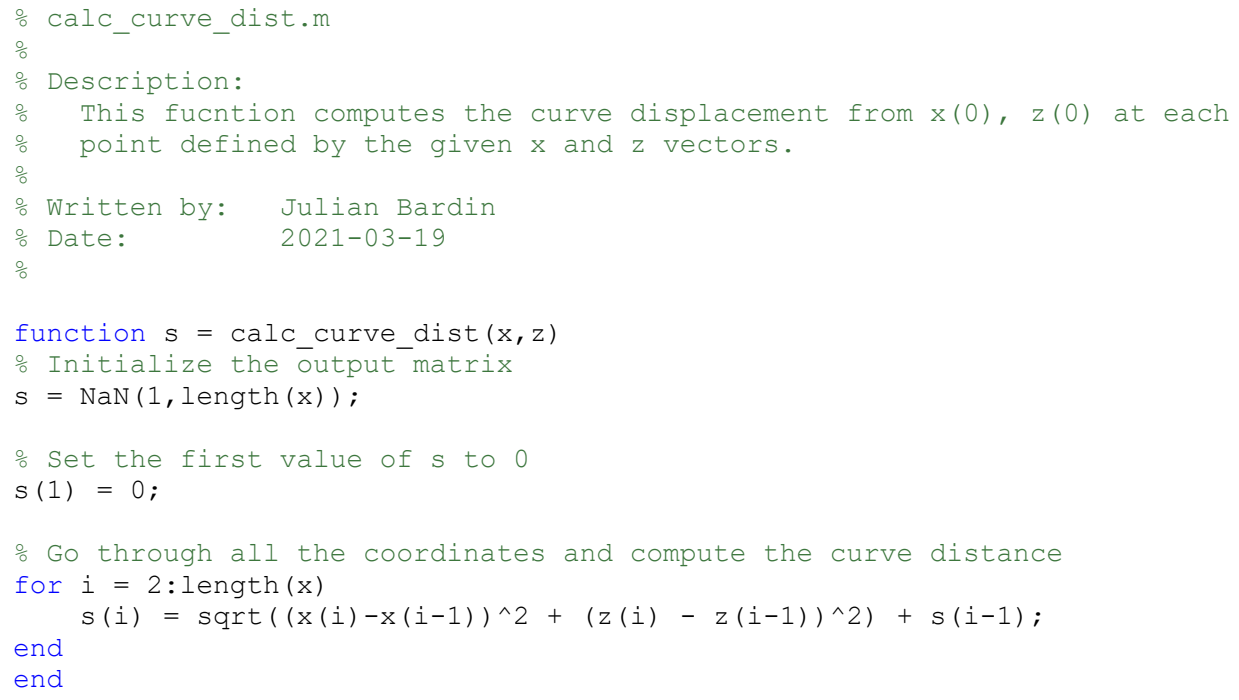




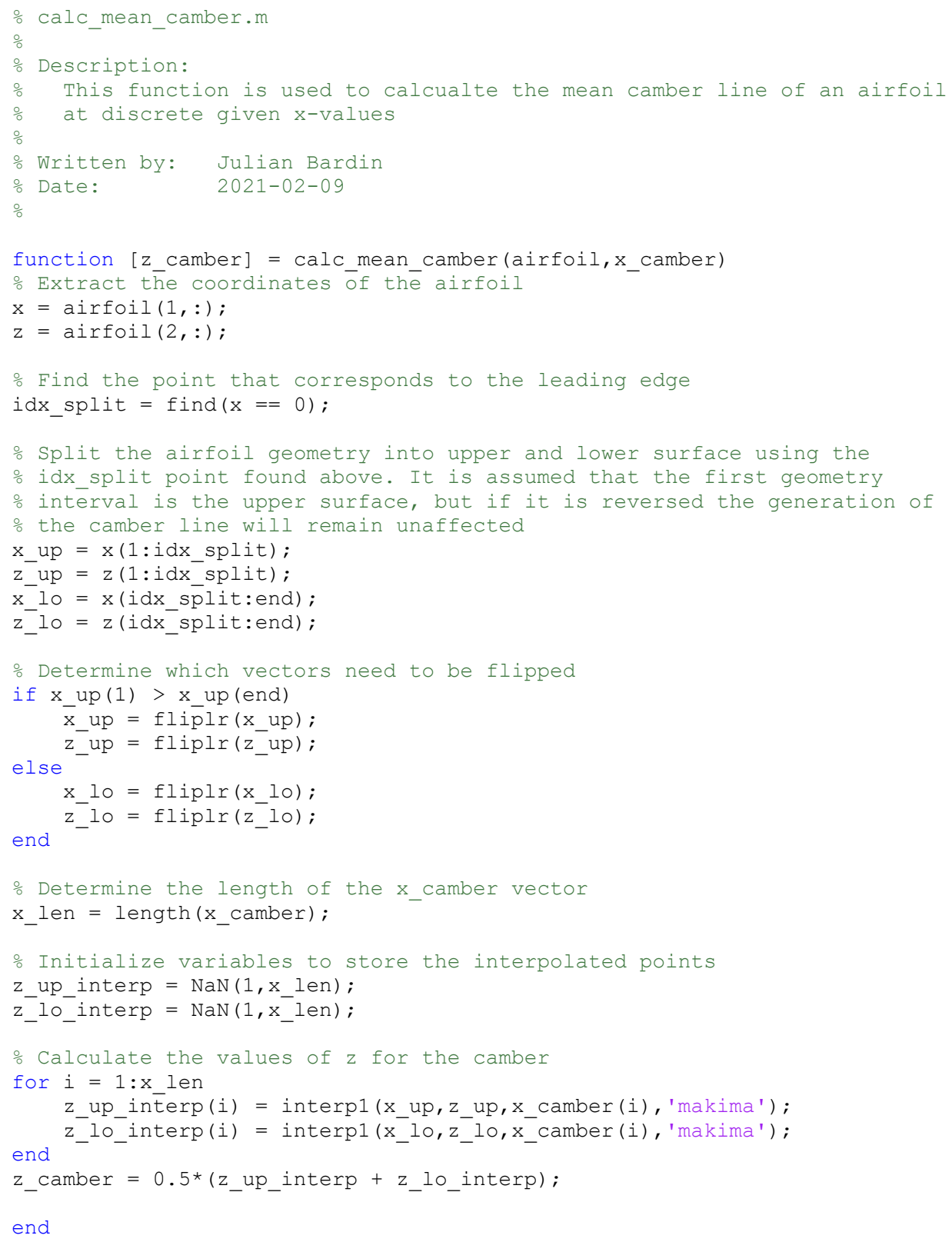




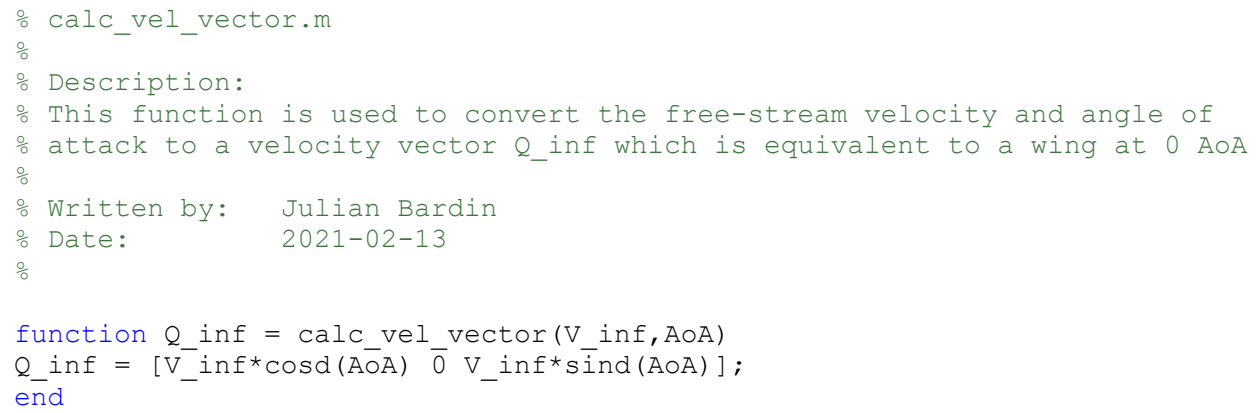




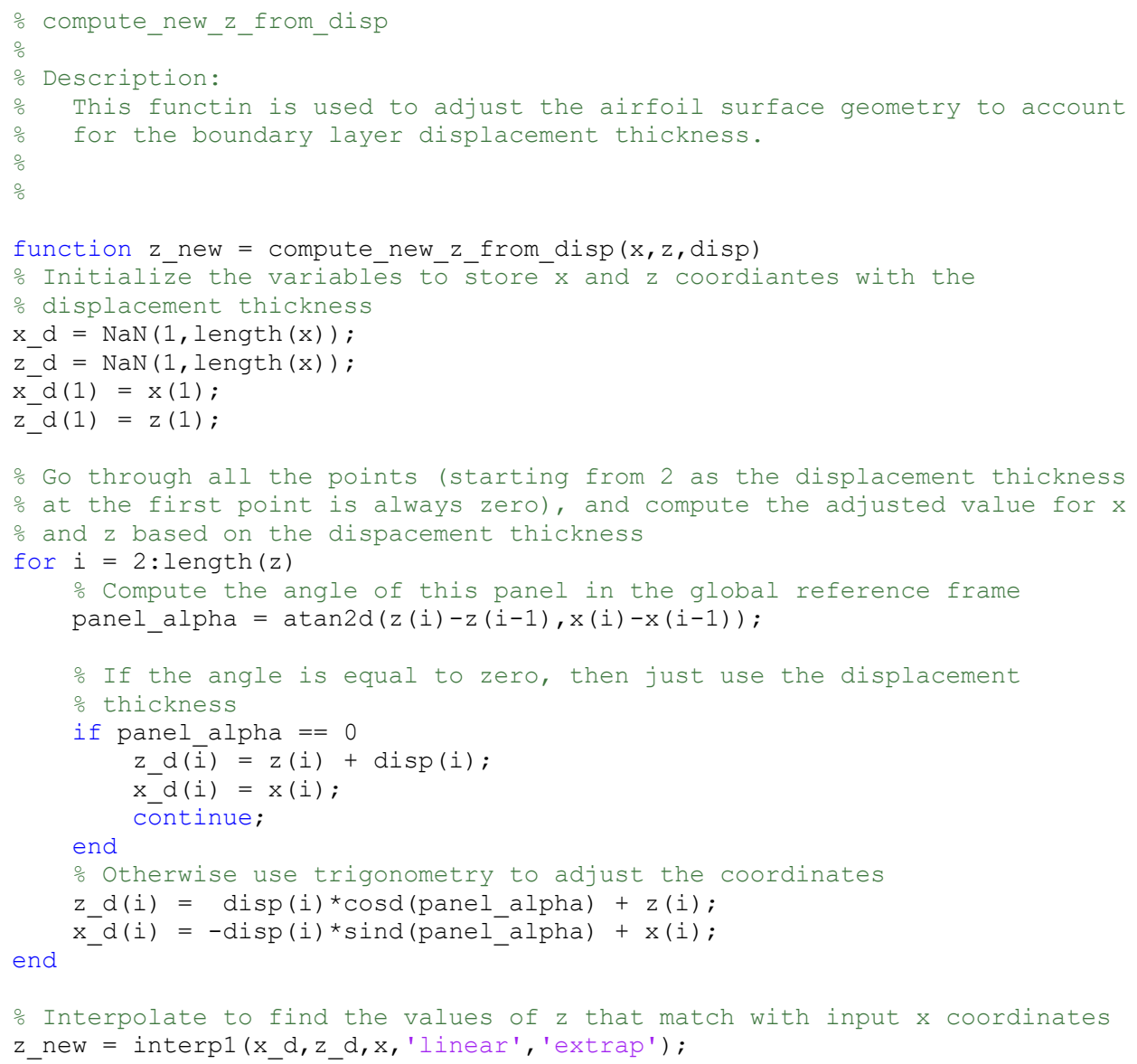




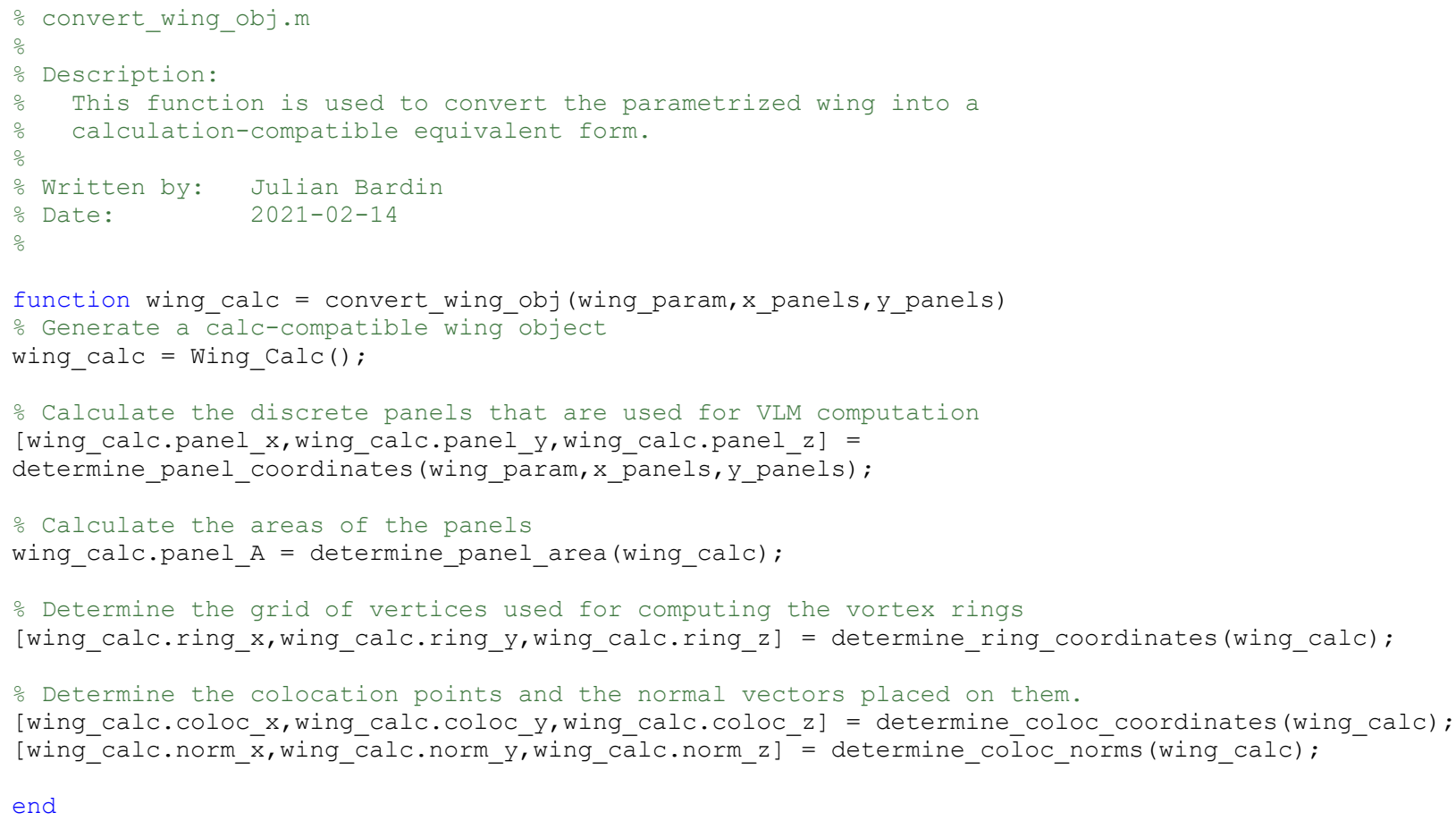




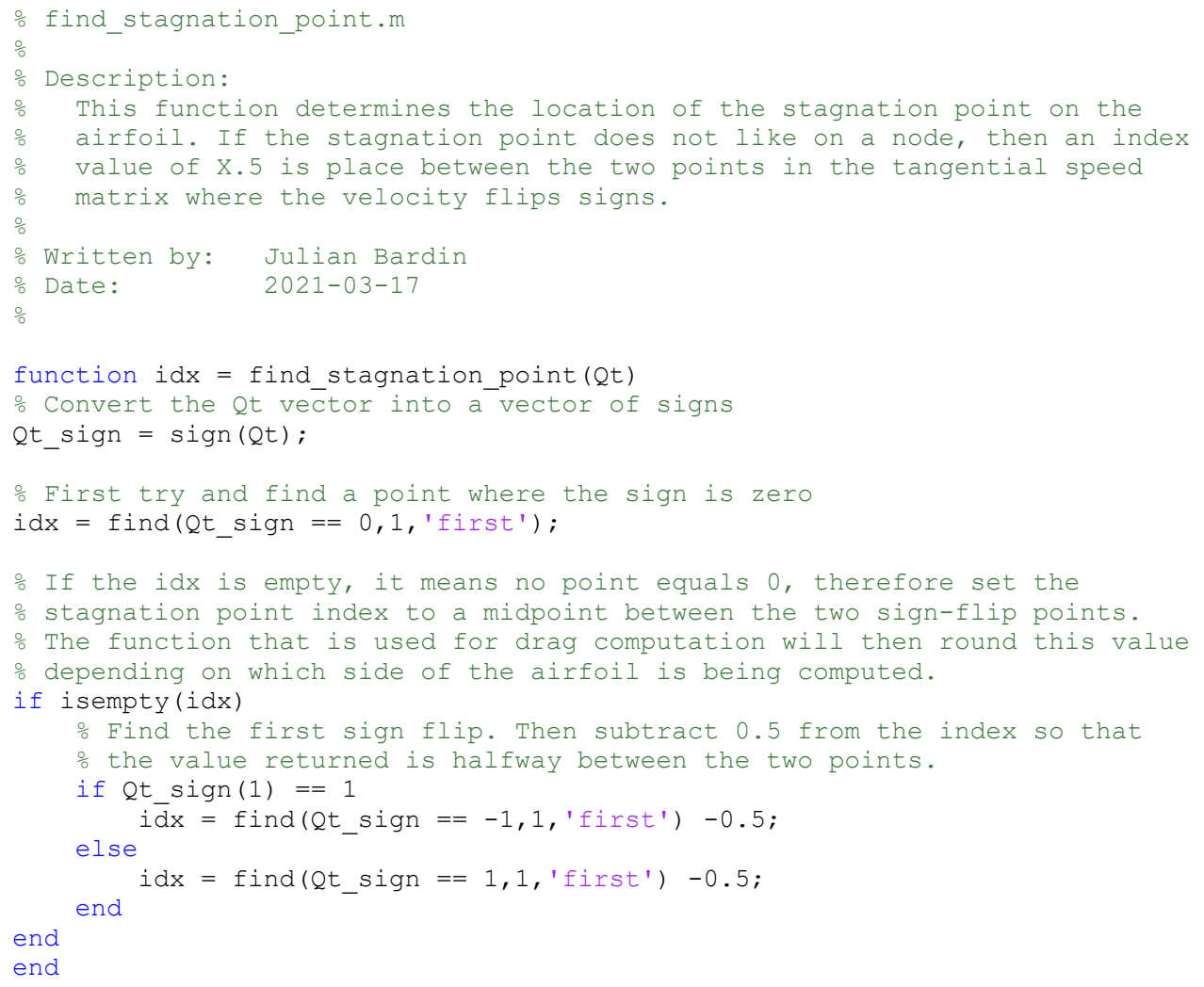




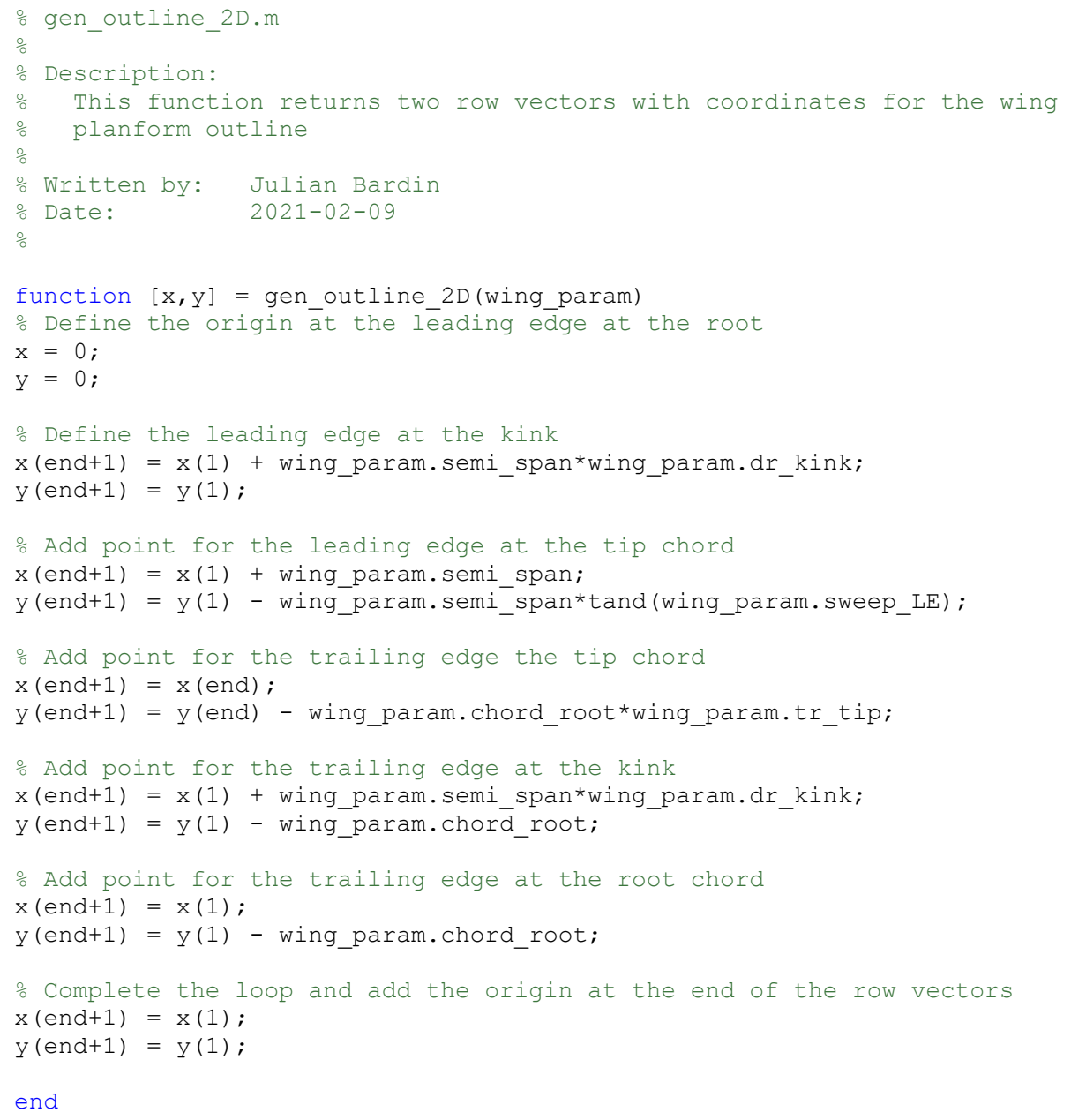




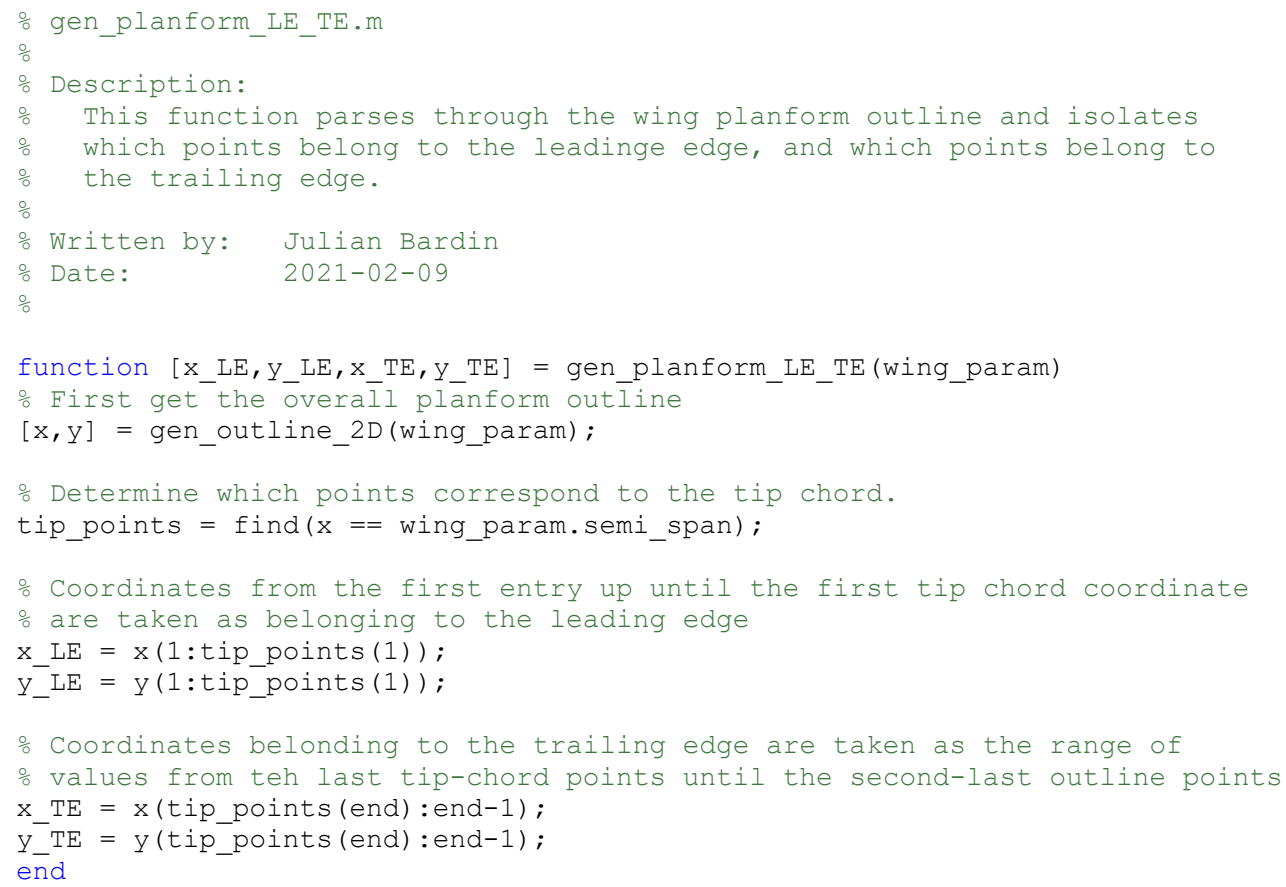




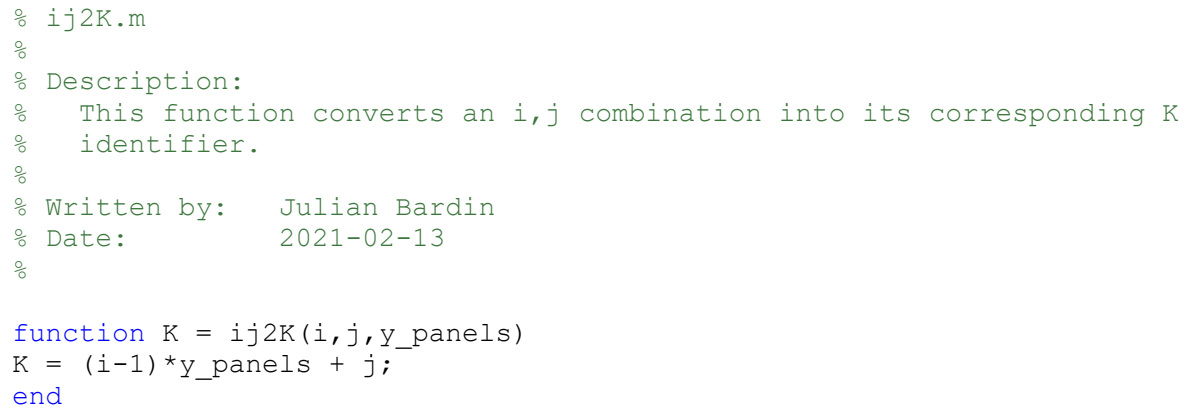




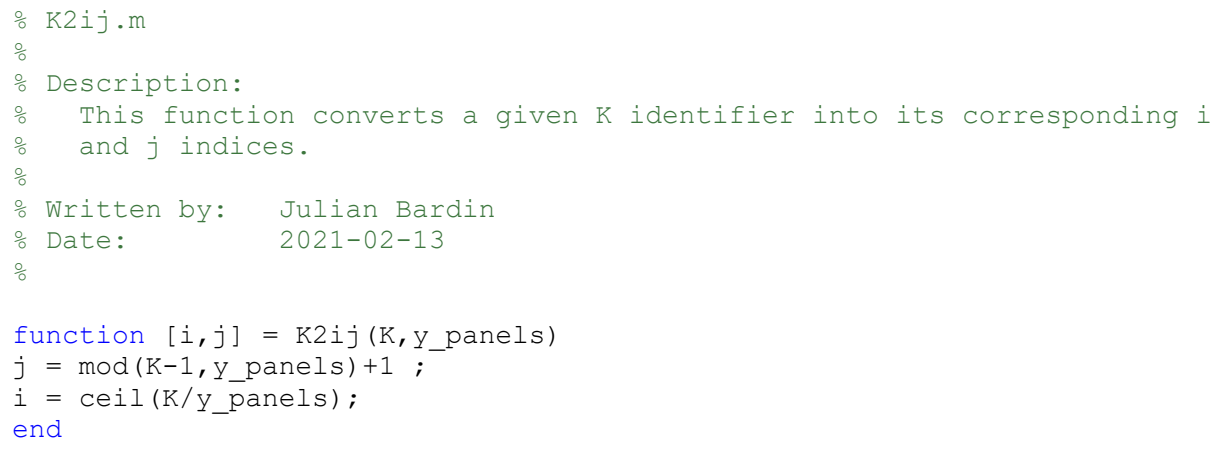




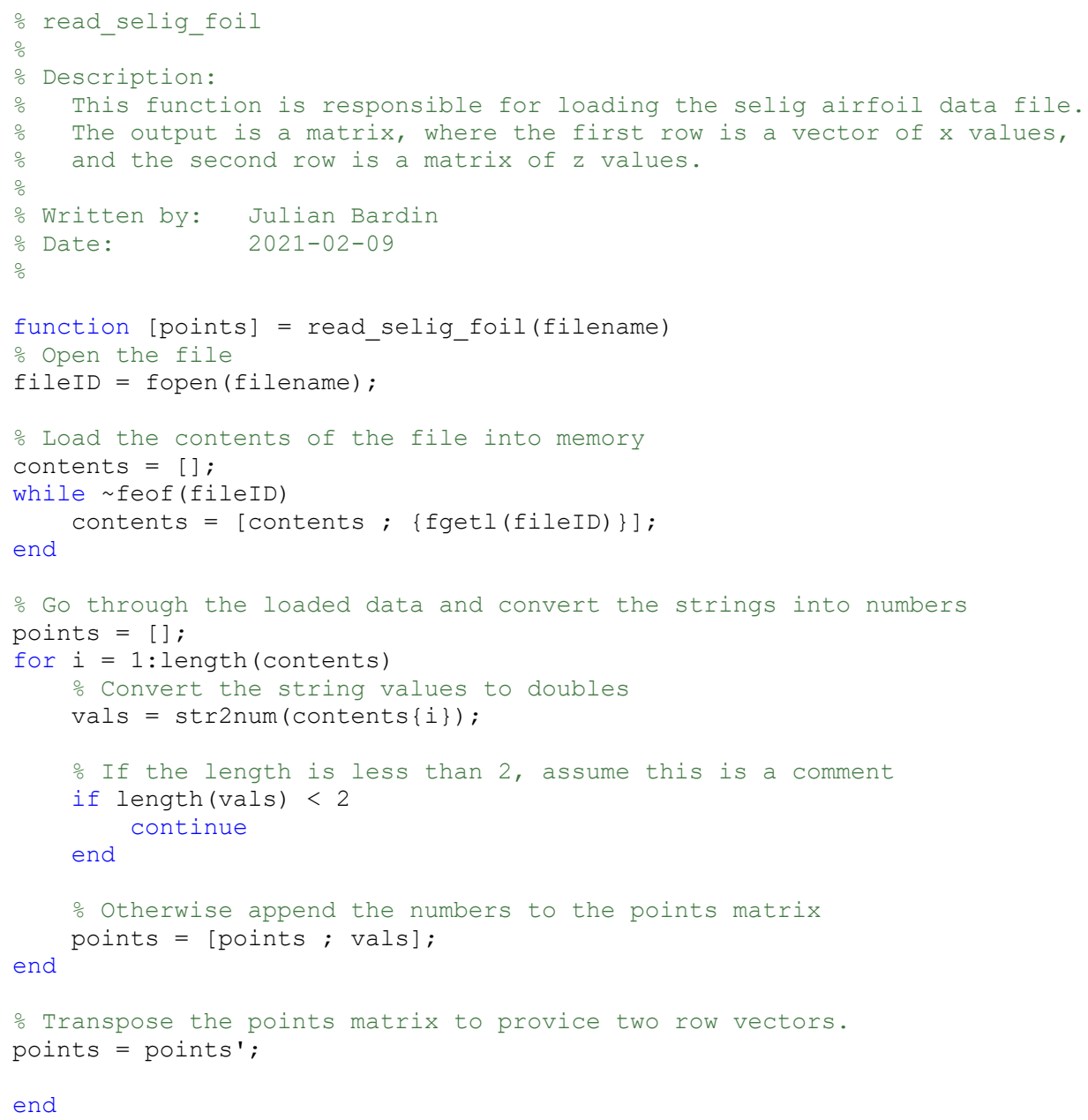




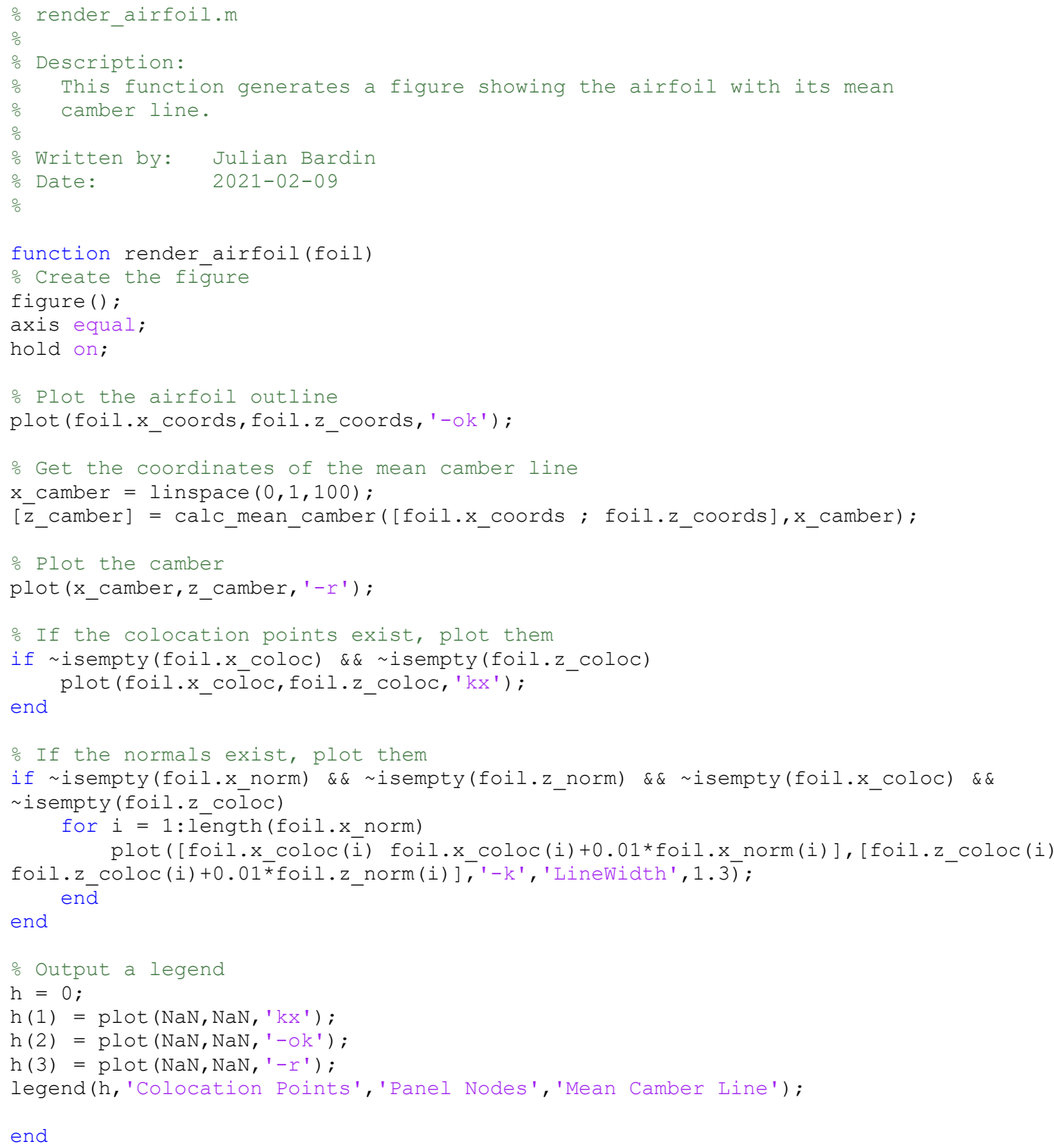




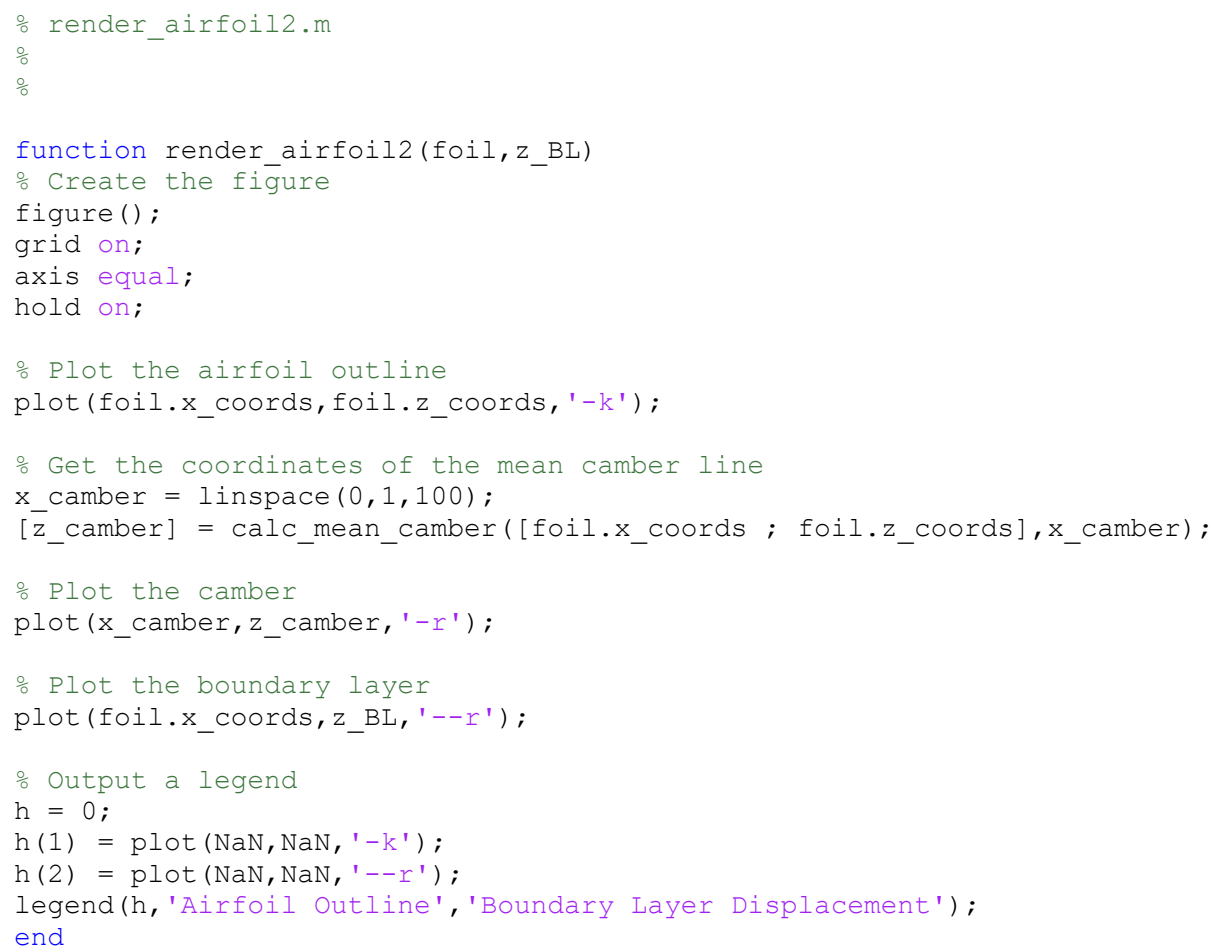




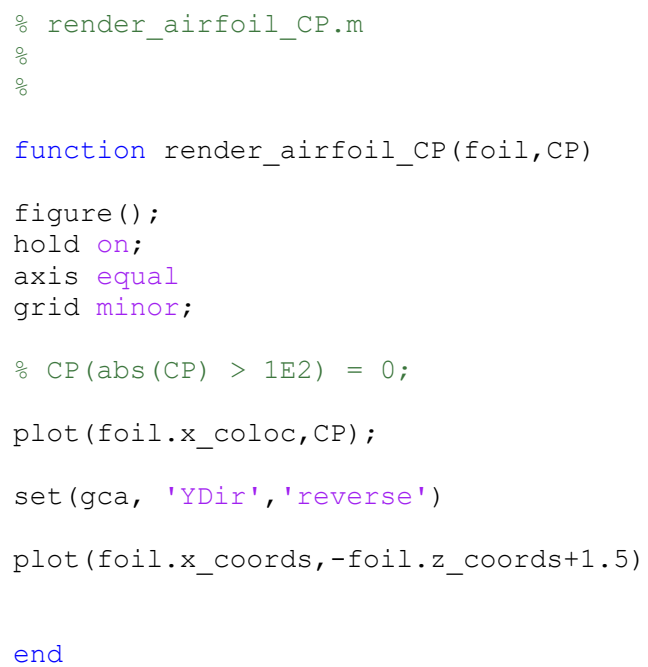




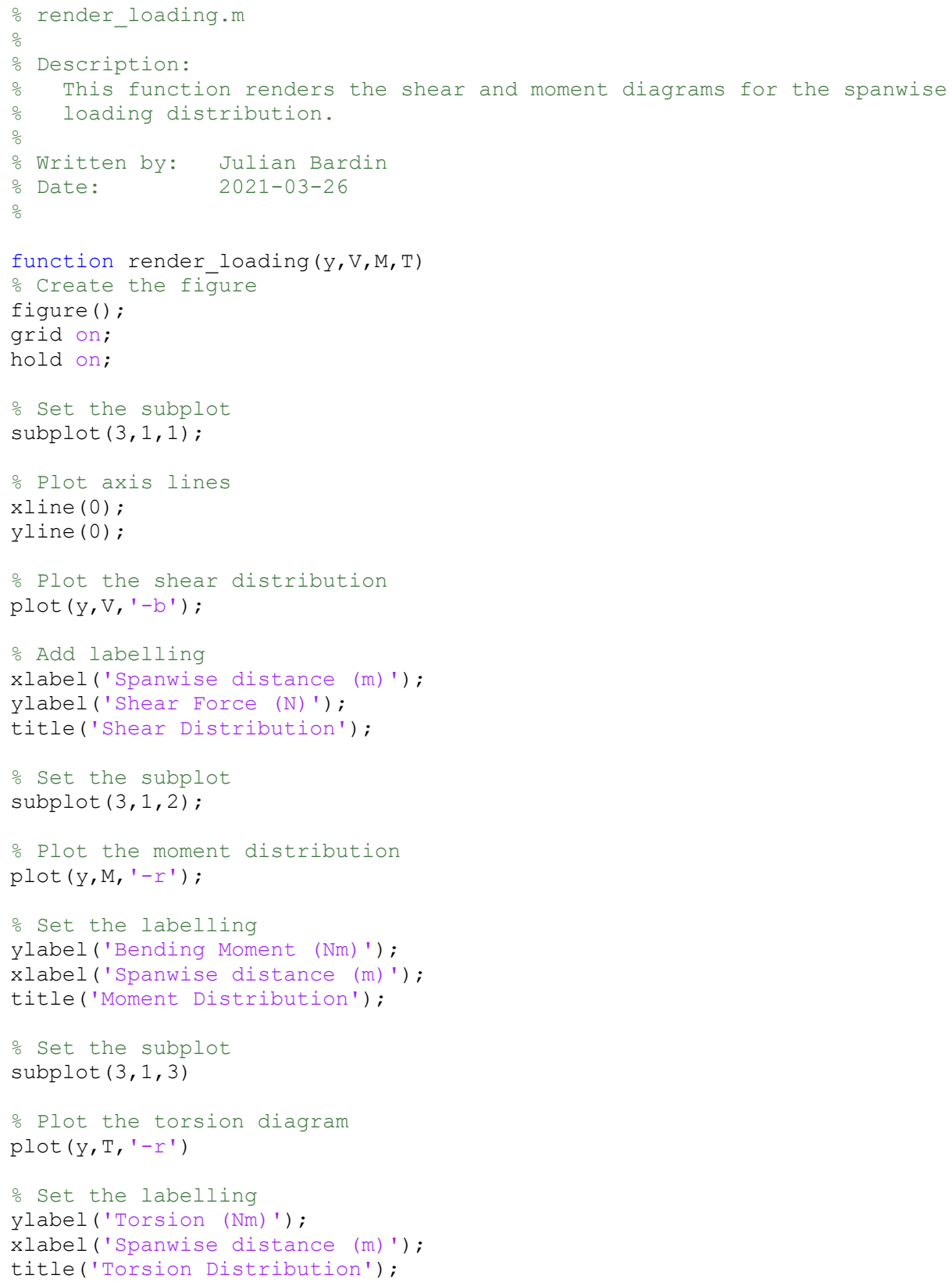

end 


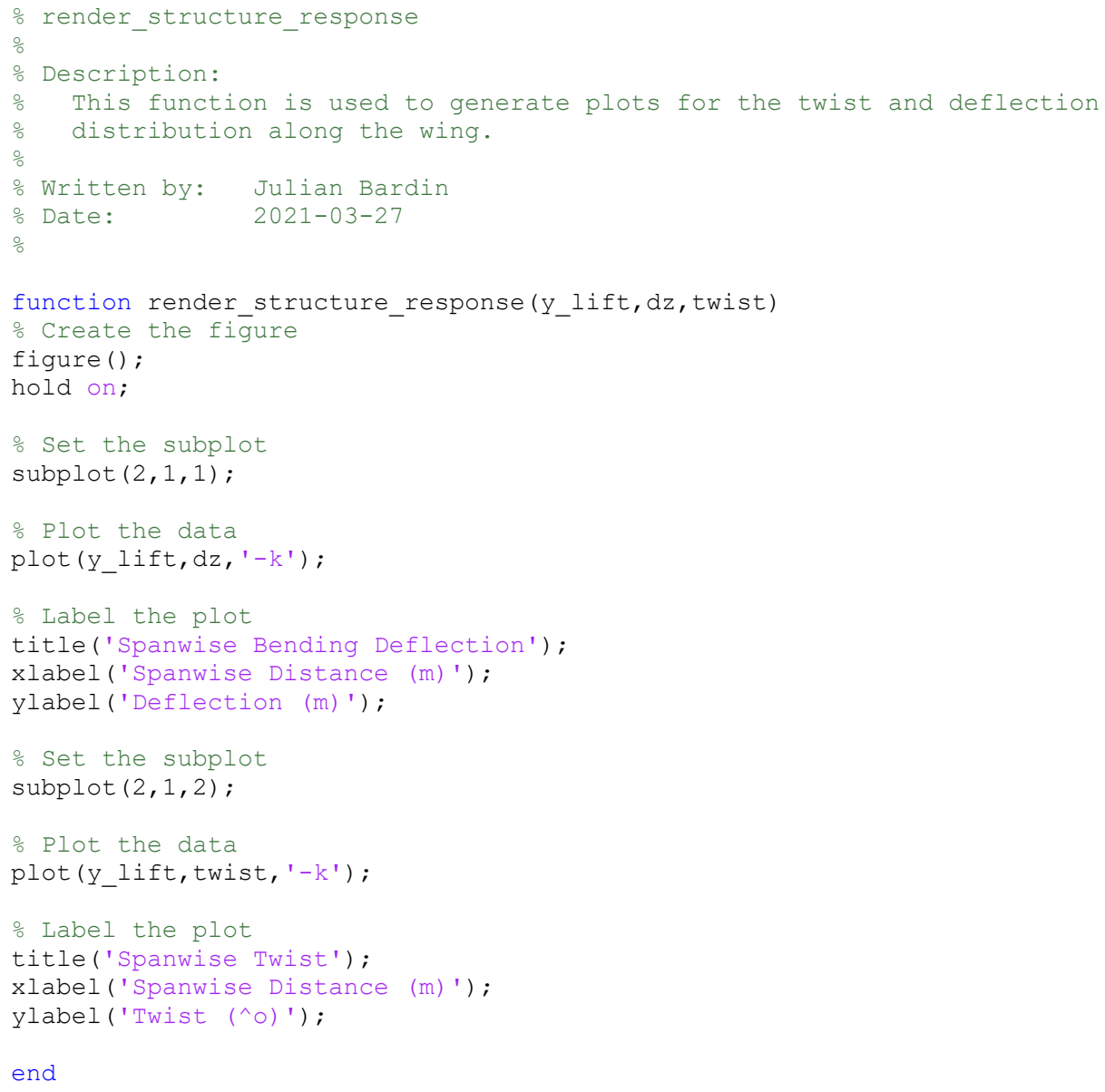




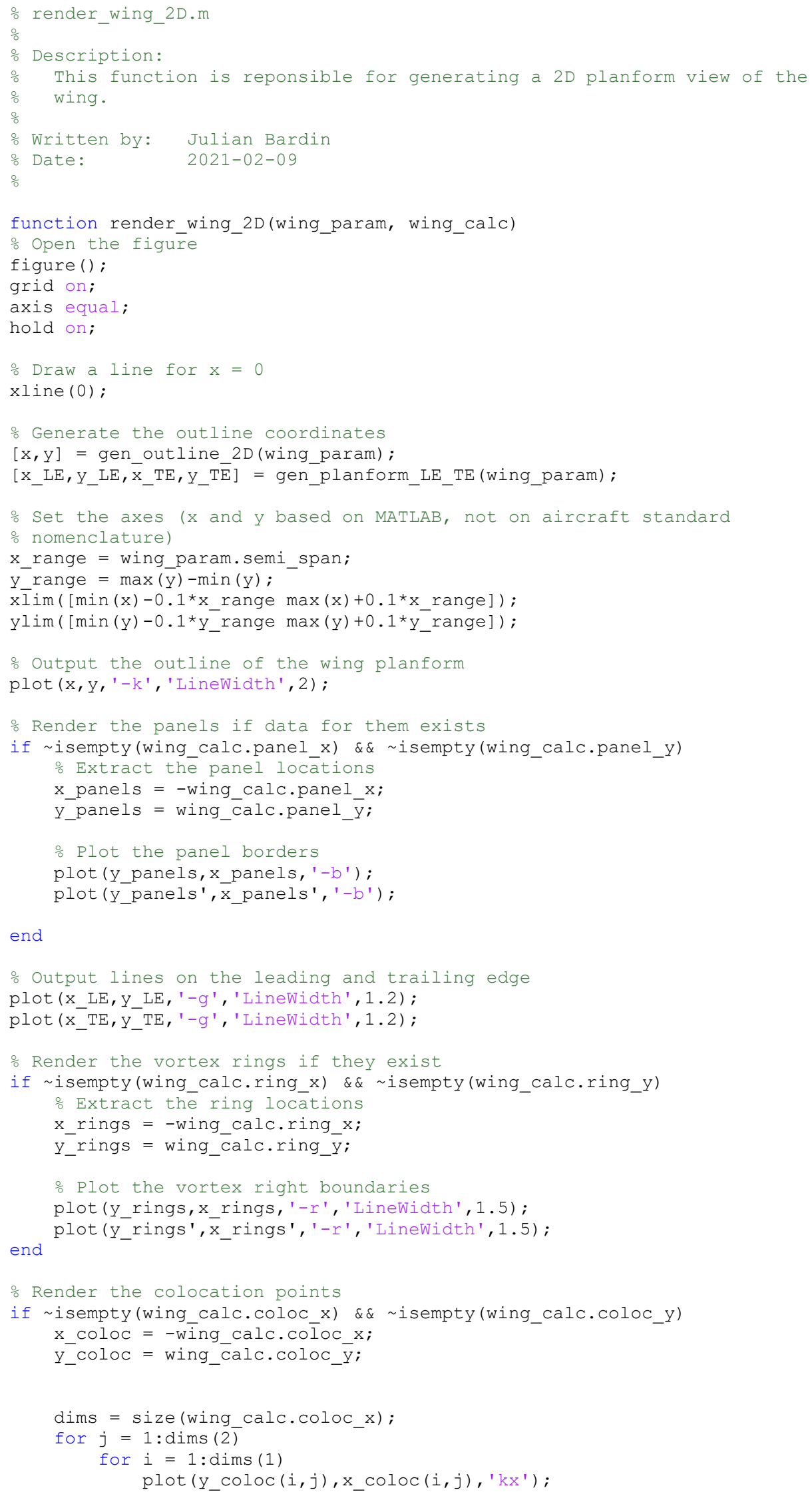




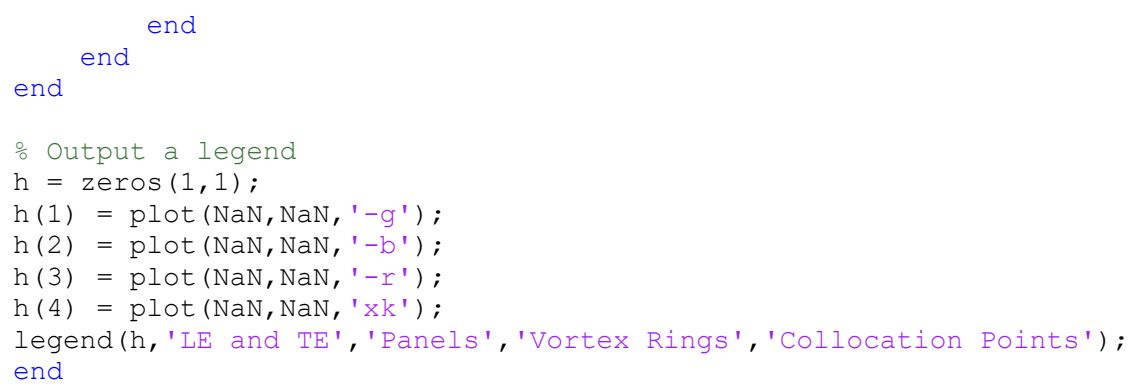




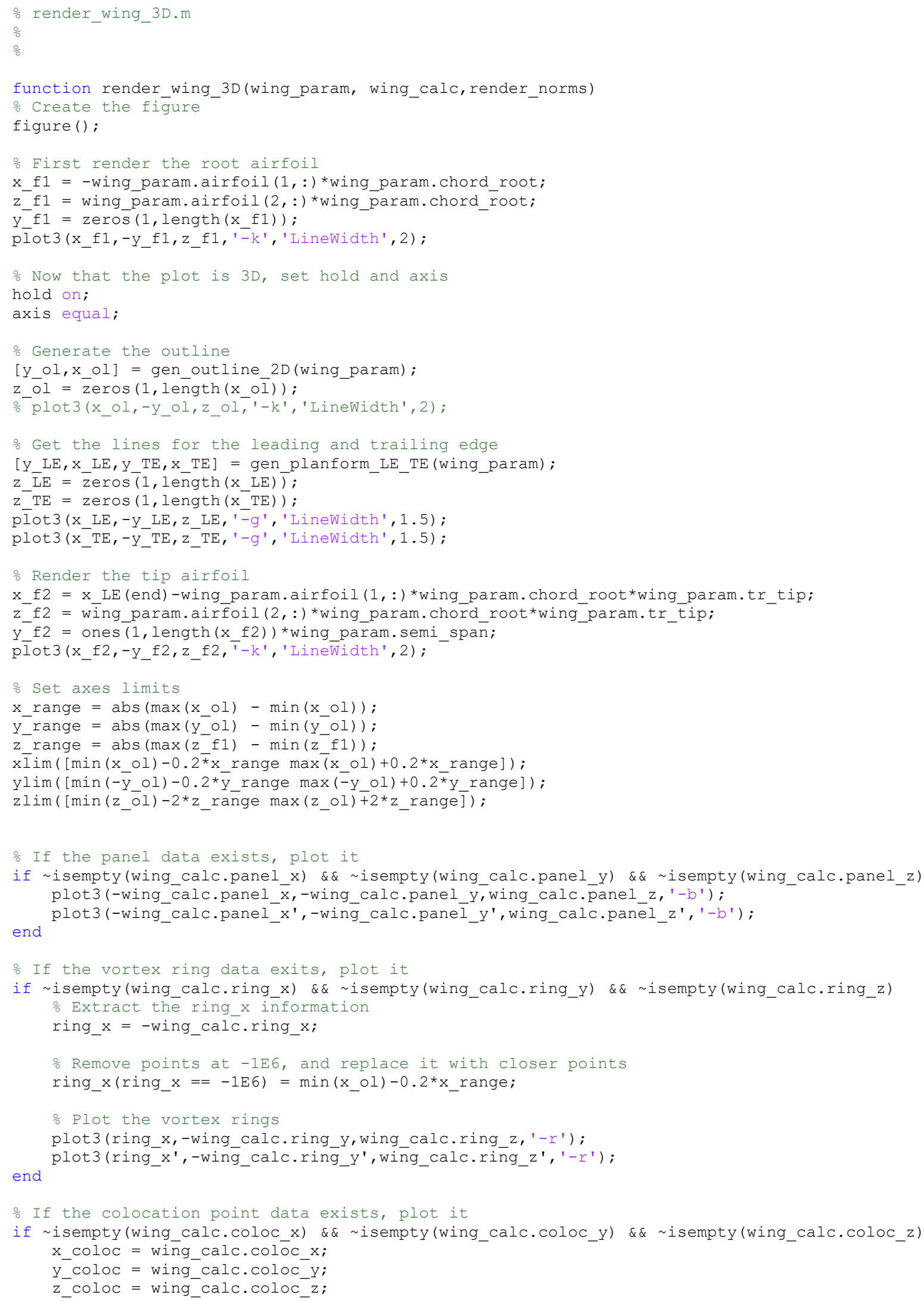




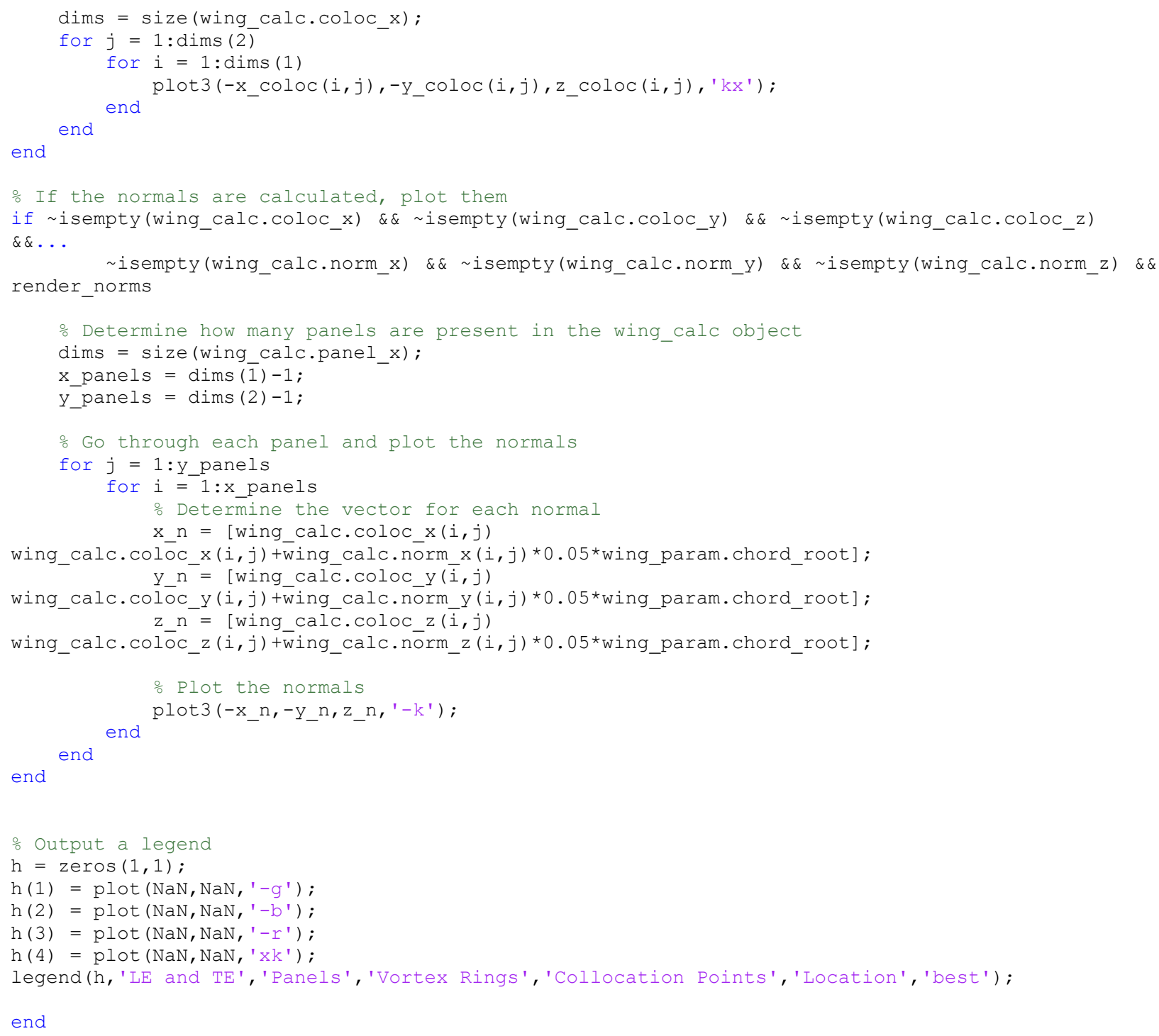




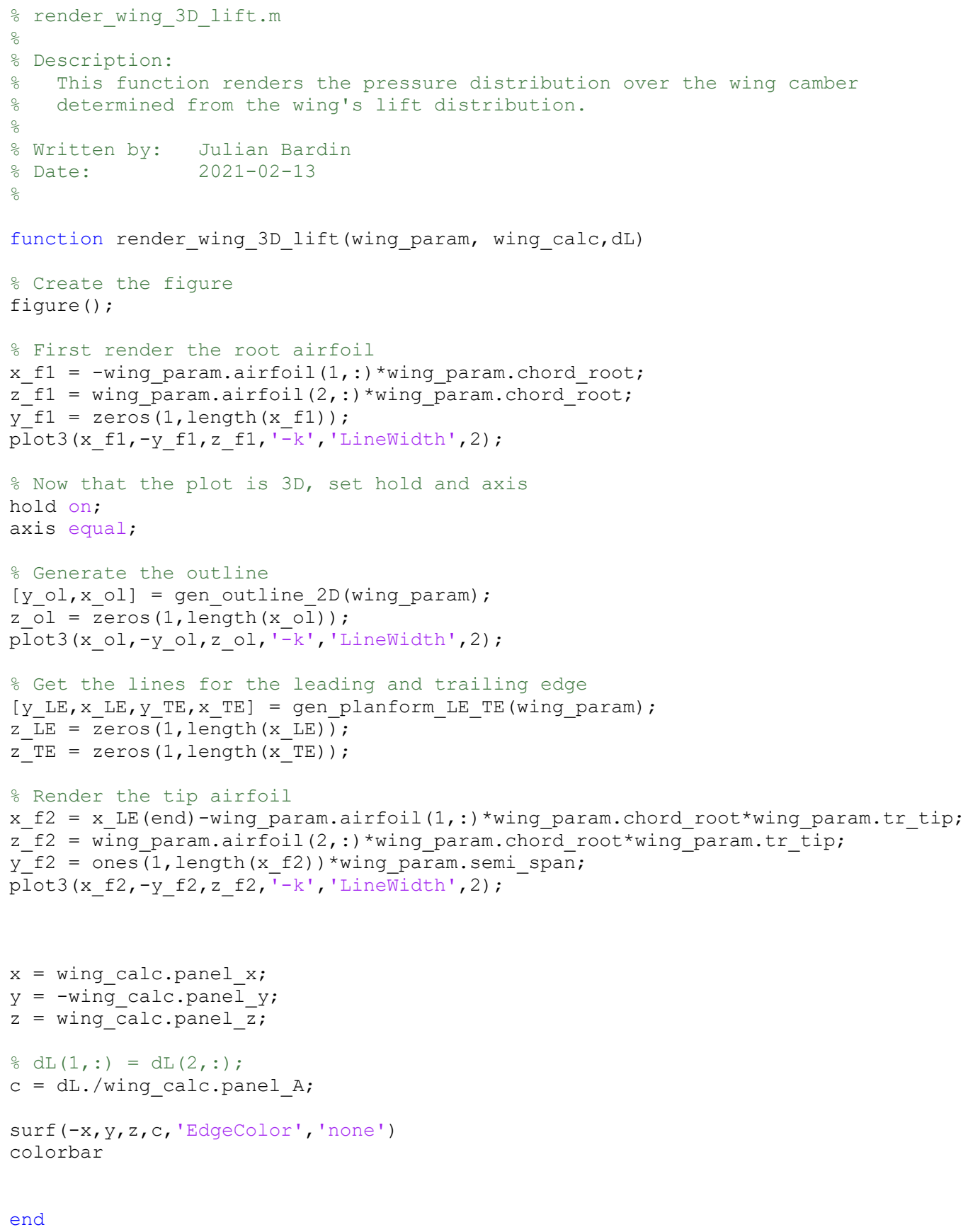




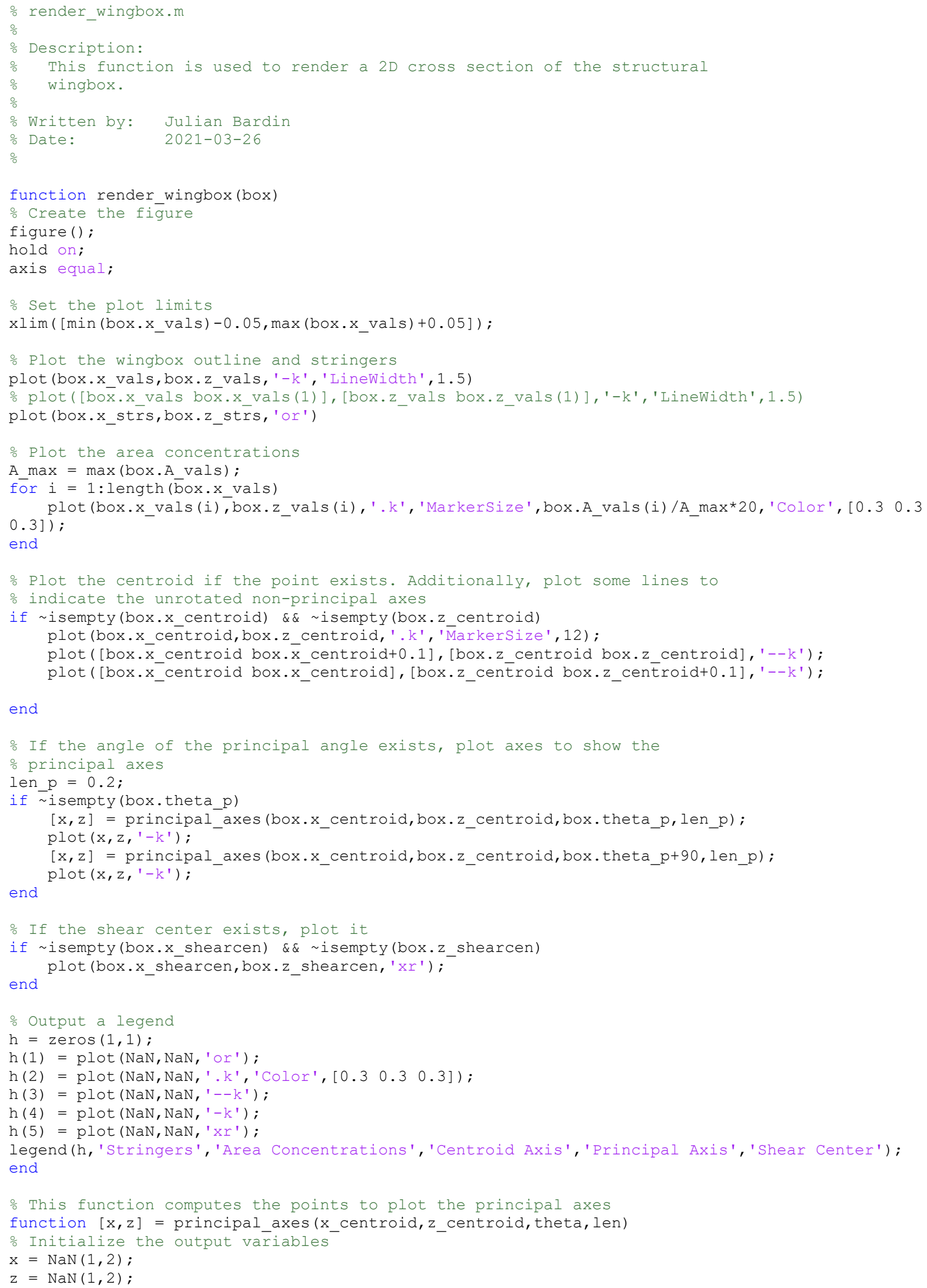


\% Set he first point to be at the centroid

$\mathrm{x}(1)=\mathrm{x}$ _centroid;

$z(1)=z$ centroid;

o Compute the following point

$m=$ tand (theta);

if theta $<-90||$ theta $>90$

else

$\mathrm{x}(2)=\mathrm{x}(1)-$ len $/ \operatorname{sqrt}\left(\mathrm{m}^{\wedge} 2+1\right) ;$

$x(2)=x(1)+1$ en $/ \operatorname{sqrt}\left(m^{\wedge} 2+1\right) ;$

end

$z(2)=m *(x(2)-x(1))+z(1)$;

end 


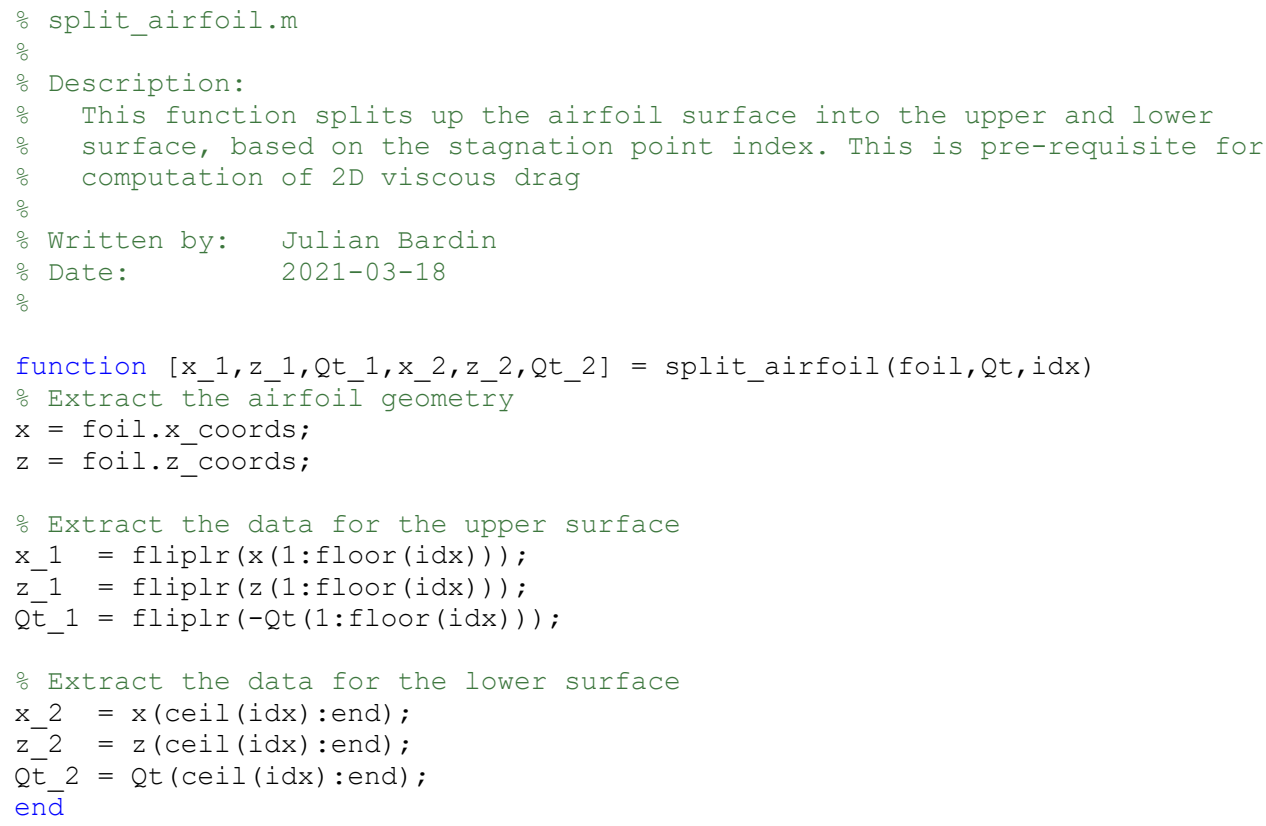




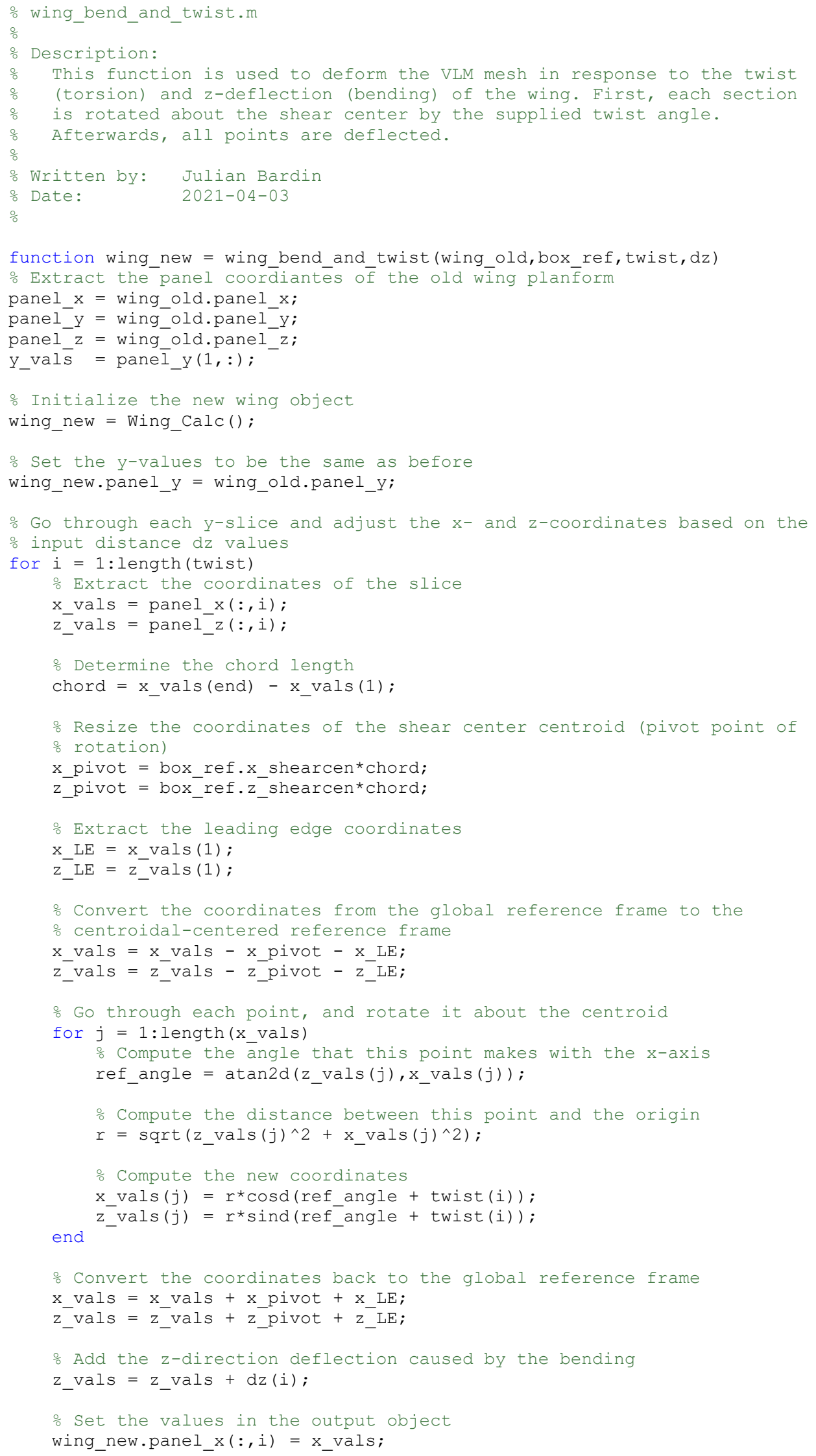


end

wing_new.panel_z(:,i) $=z_{-}$vals;

\% Calculate the areas of the panels

wing_new.panel_A = determine_panel_area (wing_new);

\% Determine the grid of vertices used for computing the vortex rings

[wing new.ring $x$, wing new.ring $y$, wing new.ring $z$ ] = determine ring coordinates (wing new);

\% Determine the colocation points and the normal vectors placed on them.

[wing_new.coloc_x,wing_new.coloc_y,wing_new.coloc_z] = determine_coloc_coordinates (wing_new);

[wing_new.norm_x, wing_new.norm_y, wing_new.norm_z] = determine_coloc_norms (wing_new);

end 


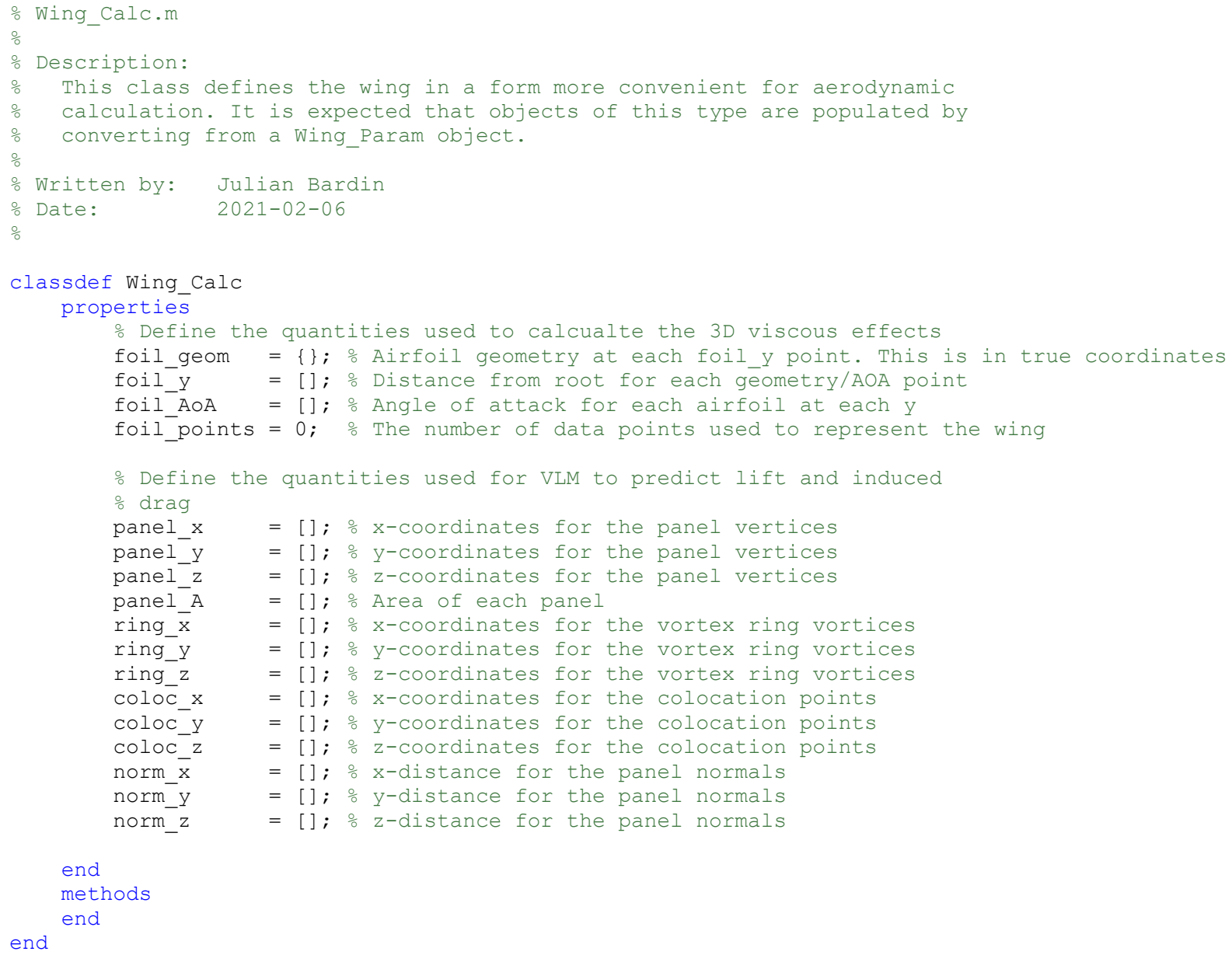




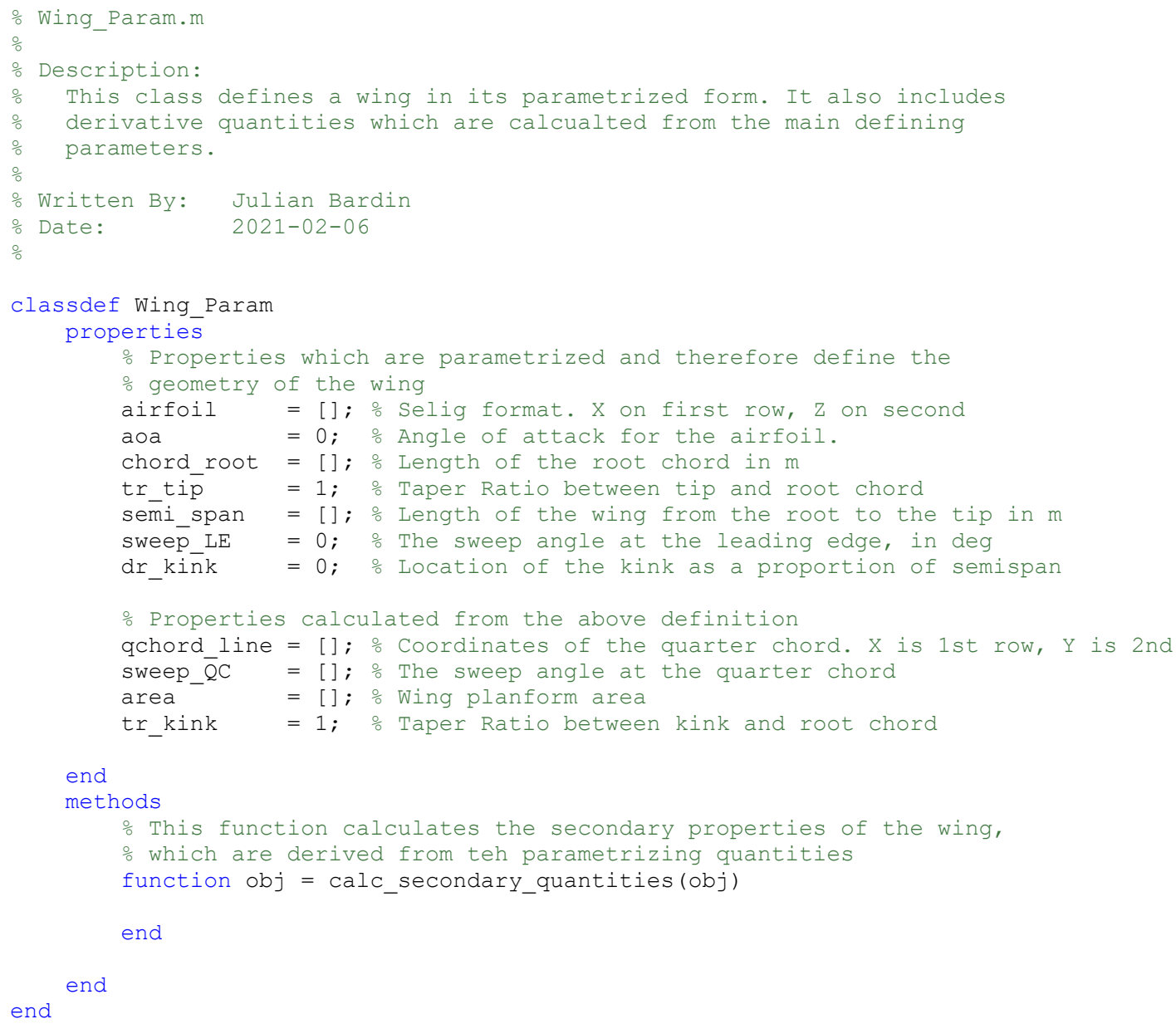




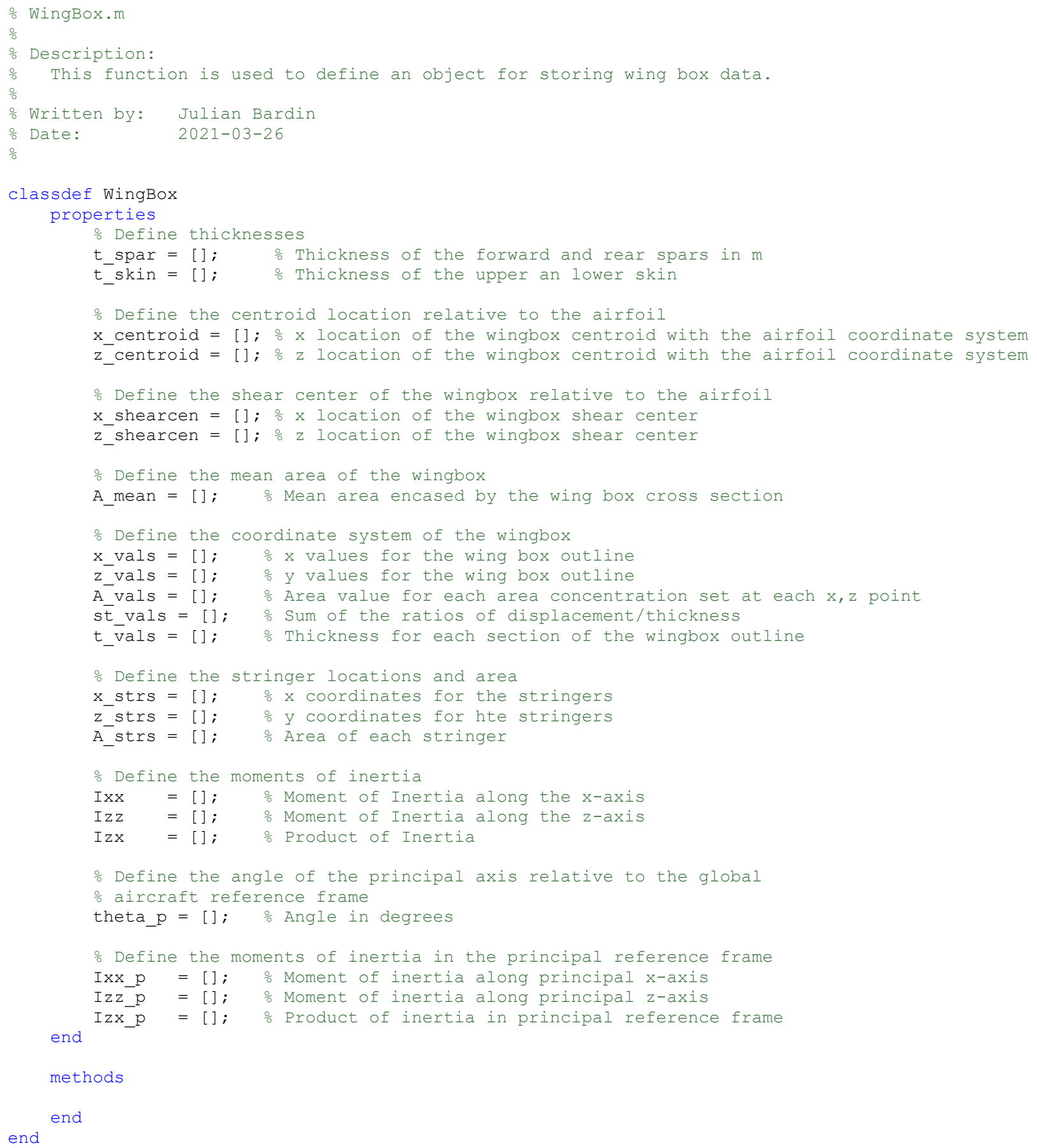

